\title{
An Interactome Map of Maize (Zea mays L.)
}

3 AAuthor information masked because of the Double-blind peer review.\} 


\section{Summary}

6 Interactomes are powerful tools for encoding and decoding complex life systems. Here,

7 we generated a maize interactome map that integrates genomic interactions,

8 transcriptomic co-expression networks, translatomic co-expression networks, and

9 protein-protein interactions throughout the maize lifecycle. This map, containing

10 over 9 million interactions in more than 5,000 functional modules, reveals extensive

11 functional divergence for duplicate genes and a progressive increase in regulatory

12 divergence between the two maize subgenomes during the flow of genetic information.

13 This network enables dissecting and validating gene functions, re-constructing

14 regulatory pathways, and deciphering molecular mechanisms underlying complex

15 traits combining big data mining technique-machine learning. By applying this map

16 to flowering-time, we identified 1,843 high-confidence genes enriched in eight

17 molecular pathways that are related to flowering time. The function of 30 (out of 58

18 tested) genes, including 27 novel genes, was verified by loss-of-function mutagenesis.

19 Furthermore, a new pathway involving histone modification was identified and

20 confirmed to regulate flowering time. The interactome map illustrates how coherent

21 sets of molecular interactions connect different types of functional elements and

22 pathway modules to map a genome-wide functional wiring landscape, which will be

23 applicable in a wide range of species.

24 Key words: Maize, Interactome, Functional genomics, Protein-protein interaction,

25 Machine learning 
Organisms and cells are inherently complex ${ }^{1}$. Although thousands of high-quality

27 genome sequences (https://wikimili.com/en/List_of_sequenced_eukaryotic_genomes) and

28 numerous genotype-phenotype associations have been uncovered using advanced

29 biological and genetic techniques ${ }^{2}$, our understanding of life systems remains limited ${ }^{3}$.

30 Interactomes are excellent tools for describing such ultracomplex systems ${ }^{4}$. Many

31 interactomes have been constructed for humans and model animals, but a few have been

32 generated for plants (http://www.pathguide.org/). A genome-wide protein-protein binary

33 interaction map was constructed for $\sim 6,200$ highly reliable interactions between $\sim 2,700$

34 proteins in Arabidopsis thaliana, revealing dramatic functional divergence of Arabidopsis

35 genes ${ }^{5}$. In addition, a quantitative atlas of the transcriptomes, proteomes, and

36 phosphoproteomes of 30 Arabidopsis tissues was recently constructed ${ }^{6}$. Despite this

37 progress, interactions that cover the genetic flow from genome to transcriptome and

38 proteome are lacking. Comprehensive interactome maps involving the genome,

39 transcriptome, translatome, and proteome simultaneously are needed to decode complex

40 life systems.

\section{An interactome map of maize}

Maize, one of the most important crops worldwide and an ideal biological and

43 genetic model, has been extensively explored for genotypic and phenotypic variations ${ }^{2}$.

44 Recently, large datasets of mRNA, protein, and phosphoprotein have been successfully

45 integrated to construct a developmental atlas for maize ${ }^{7}$. An interactome covering all layers

46 of genetic information flow would further promote the precise genetic improvement of this

47 crop. Here, we leveraged advances in high-throughput sequencing techniques to measure 
48 genomic interactions by ChIA-PET ${ }^{9}$; transcriptomic interactions for all detectable elements

49 by strand-specific RNA-seq, circRNA-seq, and small RNA-seq; translatomic interactions

50 by Ribo-seq; and protein-protein binary interactions (PPIs) by recombination-based library

51 vs. library yeast-2-hybrid (RLL-Y2H) ${ }^{8}$ analysis throughout the lifecycle of the maize

52 inbred line B73 (Fig. 1a).

Comprehensive transcriptomic and translatomic deep sequencing generated 1.78

54 trillion sequencing bases (Supplementary Fig. 1; Supplementary Table 1). Transcriptomic

55 profiling uncovered the expression of 37,701 protein coding genes ( $9.5 \%$ of the genome),

569,858 long non-coding RNAs (lncRNAs) (1.4\%), 61,137 circRNAs (2.3\%), 343,507 small

57 RNA (sRNA) clusters (67-87\% using different gap thresholds), and 145 fusion expressed

58 elements $(0.001 \%)$ across 26 different stages and tissues (Fig. 1b; Supplementary Fig. 1).

59 For translatomic profiling, we performed Ribo-seq ${ }^{10}$ on 20 out of the 26 tissues and

60 identified 34,949 genes that were embedded in ribosomes. These expressed elements at

61 both the transcriptome and translatome levels span $90.5 \%$ of the reference genome,

62 pointing to pervasive transcription in maize.

63 We conducted a series of multi-omic experiments to dissect interactions of any pair 64 of functional elements and functional modules, where functional elements with similar 65 roles were clustered coherently (see Methods). At the genome level, 13,142 loci formed $66 \quad 14,788$ 3-D physical interactions within 689 modules $^{9}$ (Fig. 1c). At the transcriptome level,

67178,025 expressed elements coherently functioned in 2,944,080 co-expression pairs for 685,998 modules (Fig. 1d; Supplementary Table 2). At the translatome level, 23,646 genes 69 formed 68,972 translational co-expression pairs in 1,485 modules (Fig. 1e; Supplementary 70 Table 2). 
To construct a genome-wide protein-protein interactome (PPI), we used eight bait

72 and eight prey cDNA libraries from eight distinct tissues across the whole developmental

73 stages and to screen for interactions via 130 library vs. library matings using the RLL-Y2H

74 system. We performed Pacific Biosciences (PacBio) Sequel sequencing

75 (https://www.pacb.com/) of plasmids isolated from the surviving positive yeast clones

76 from each mating (Supplementary Fig. 3; Supplementary Table 3). We obtained nearly 9

77 million consensus PacBio full-length sequences and identified 87,775 high-confidence

78 PPIs between 9,386 Binding Domain (BD) bait genes and 16,477 Activation Domain (AD)

79 prey genes, filtered from over 360,000 detectable PPIs (Fig. 1f, Supplementary Table 4).

The multi-omic interactomes exhibited markedly different topological hierarchy

81 (Fig. 1c-1f). Based on different confidence cut-offs (See Methods), the interactomes were

82 grouped into low-, middle-, and high-confidence versions that showed similar trends

83 (Supplementary Fig. 3). The high-confidence interactome was used for subsequent

84 analyses. Although a subset of hub nodes (functional elements) from different -omics data

85 overlap (Fig. 1g; Supplementary Fig. 4), the overall clustering coefficients was 0.254 in

86 the $3 \mathrm{D}$ genome, 0.321 in the transcriptome, 0.12 in the translatome, and 0.006 in the

87 proteome (Supplementary Table 2), exhibiting a distinct topological structure for each

88 regulatory layer (Fig. 1c-1f).

We integrated the interaction network from different layers. In all, we generated

90 over 2 trillion bases and detected over 182,995 functional elements and ultimately

91 constructed an interactome with over 3 million edges (Fig. 1h; Supplementary Fig. 5).

92 Diverse types of regulatory elements interacted in this network, for example, teosinte

93 glume architecurel (tgal) ${ }^{11}$ showed interactions with lncRNAs, circRNAs, and miRNAs 
94 which were not found previously (Fig. 1i). We've developed a user-friendly website to store

95 all the interactome information (http://minteractome.ncpgr.cn/index.php), which can be

96 searched easily by gene name or using node information.

97 A comparison of our interactome with previously generated interactomes revealed 98 significant conservation of interactions (Supplementary Fig. 6a-6j), and over 50\% of PPIs 99 could be validated using other biological techniques (Supplementary Table 5; 100 Supplementary Fig. 6k-6n; Supplementary Fig. 7). Although it is still not saturated 101 (Supplementary Fig. 6o), this is the largest protein interaction database to date $(\sim 360,000)$, 102 and the interactome map represents the largest, nearly complete, high-quality interactome 103 in plants ${ }^{5,7}$. 

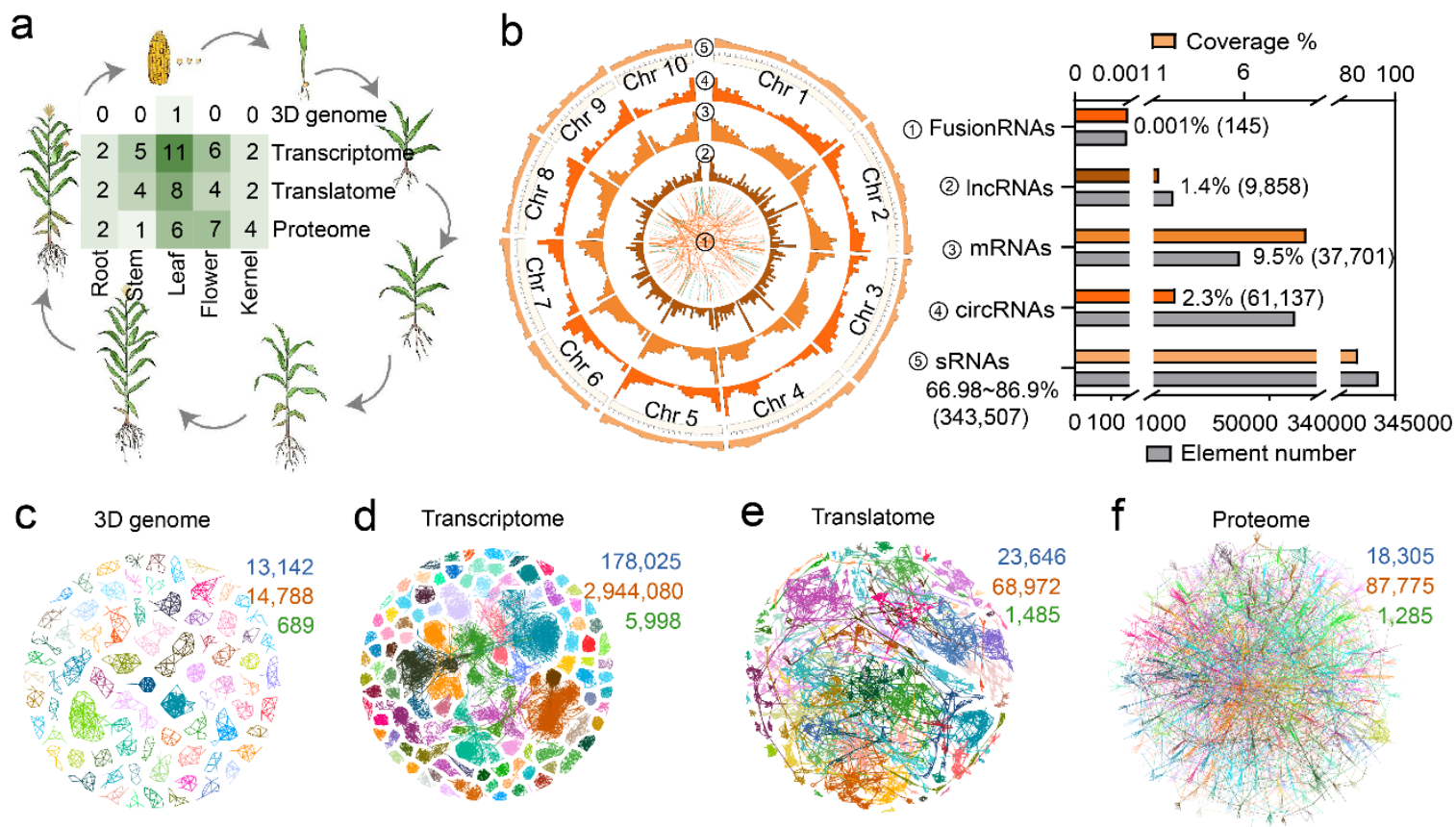

g

$$
9
$$

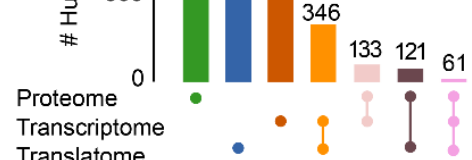

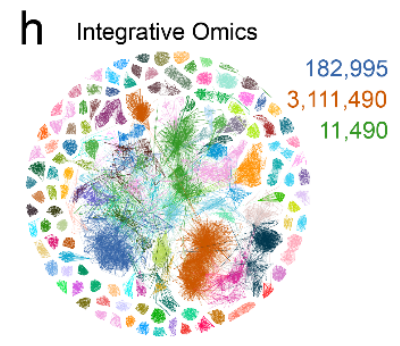

i

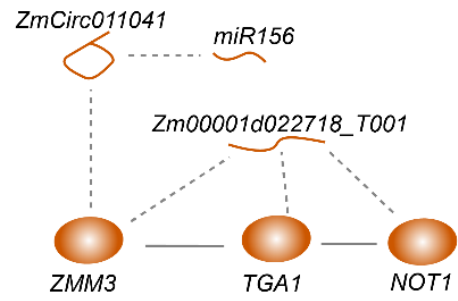

Figure 1. The interactome uncovers the genome-wide regulatory landscape in maize. a, Data collection. Complete omics data covering all layers of genetic information flow were collected from maize inbred B73 throughout its entire lifecycle. The numbers in the cells of the matrix indicate the number of tissues collected for each of the four types of high-throughput data. b, Distribution and proportion of different types of expressed RNAs across the maize genome. c$\mathrm{f}$, Genome-wide landscape of interactions in the genome, transcriptome, translatome, and proteome, respectively. Numbers in each panel (from top to bottom) represent the number of functional elements, interactions (edges), and modules. g, Total number of hub genes and number of overlapping hub genes (nodes above the 90th percentile for the number of edges) among the transcriptome, translatome, and proteome. h, Genome-wide landscape of integrative interactome based on multi-omics. i, An example of an interaction module for the well-known gene tgal involving IncRNAs (orange long line), circRNAs (orange circle), small RNAs (orange short line), and protein-coding mRNAs (orange oval). Solid and dotted lines represent direct and indirect interactions, respectively. 


\section{Progressive functional divergence between the maize}

\section{8 subgenomes from DNA to proteins}

The interactome map reveals dramatic regulatory divergence for duplicated genes

120 as expected (Supplementary text; Supplementary Fig. 8). Notably, maize is an

121 allotetraploid with distinct ancient subgenomes, Maize1 and Maize2, that exhibit

122 asymmetric divergence in both gene content and expression levels ${ }^{12}$. Although Maize1, the

123 dominant subgenome, contains more orthologs and highly expressed genes than Maize2,

124 there is no evidence for regulatory bias between the two subgenomes ${ }^{12,13}$. We detected no

125 or only subtle differences between the subgenomes at the transcriptome level. However,

126 significant differences were detected at both the translatome and proteome levels (Fig. 2a;

127 Supplementary Fig. 8g-8h). We aligned the maize genome with its ancestral sorghum

128 genome and quantified the overall degree of differentiation of genes in a sliding window

129 (100 genes). The regulatory divergence of the subgenomes increased from transcriptome

130 to translatome to proteome and exhibited dramatic variation across different windows (Fig.

131 2b; Supplementary Fig. 9-10).

To further explore the evolutionary relevance of subgenome divergence, we

133 focused on hub genes at different omic layers. Intriguingly, Maize1 hub genes of both the

134 transcriptome and translatome levels were significantly over-represented $(P<0.01)$ at

135 genomic regions targeted for domestication and improvement ${ }^{14}$; however, Maize1 hub

136 genes of the proteome level were not over-represented (Fig. 2c). Accordingly, Maize2 hub

137 genes of the transcriptome level were significantly enriched $(P<0.001)$ in loci targeted for

138 domestication and improvement, and Maize2 hub genes of both the translatome and 
140 Supplementary Fig.11). These results indicate that the variation of Maize1 hub genes at

141 both the transcriptome and translatome levels and of Maize2 hub genes at the

142 transcriptome-level might be important for speciation and crop improvement. The non-

143 enrichment of hub genes in neither subgenomes suggests that selection based on protein-

144 coding sequence variation of maize hub genes is risky probably due to the detrimental

a
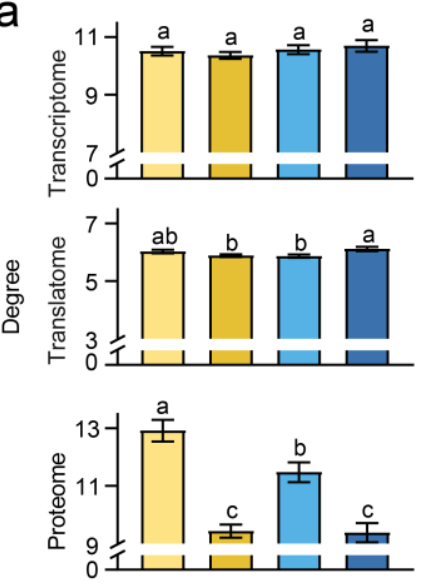

Maize1 Maize1 without maize2

Maize2 Maize2 without maize1
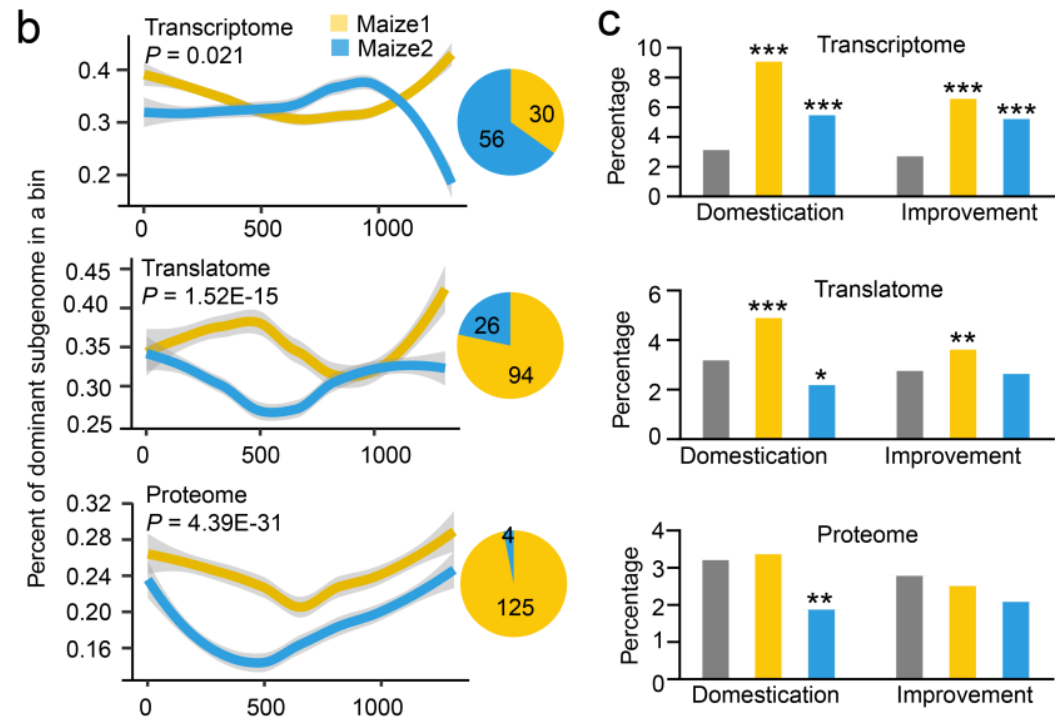

Figure 2. Asymmetric regulatory divergence indicates differences in evolutionary selection between the two maize subgenomes. a, Degree of variation of genes in subgenomes Maize1 and Maize2 across different regulatory levels. Maize1 and Maize2 represent homologous genes from subgenome Maize1 and Maize2, respectively. Maize1 without Maize2 indicates Maize1 genes whose corresponding Maize2 genes were lost. Maize2 without Maize1 indicates Maize2 genes whose corresponding Maize1 genes were lost. b, Biased regulatory fractionation at the transcriptome (top), translatome (middle), and proteome (bottom) levels is observed for the reconstructed or "sorghumized" pair maize ancestral chromosome-chromosome 1. Dominant bin is defined as a genome region in which more genes in one subgenome have a significantly higher degree of bias than the corresponding homologous genes in the other subgenome. Lines are LOESS regression lines. The pie chart to the right represents the number of dominant bins from each subgnome. c, Enrichment of Maize1 and Maize2 hub genes at the transcriptome (top), translatome (middle), and proteome (bottom) levels at loci targeted for domestication and improvement. *, **, and *** indicate $\mathrm{P}$-values $<0.05,0.01$, and 0.001 by Chi-square test, respectively. Grey, yellow, and blue bars represent genome-wide, Maize1, and Maize2, respectively. 
The interactome map can reconstruct the networks of well-known genes and uncover new crosstalk genes with similar function. We explored an interaction subnetwork 163 involving three key maize tillering genes, Teosinte Branched $1(\text { TB1 })^{16}$, Grassy Tillers 1 $164(G T 1)^{17}$, and Tassels Replace Upper Ears $1(T R U 1)^{18}$. Loss-of-function mutations in any of 165 these three genes lead to more tillers, a characteristic of the maize ancestor teosinte. TB1, 166 GT1, and TRU1 demonstrated an interaction with ZmALOG1 (Zm00001d003057) and 167 ZmALOG2 (Zm00001d032696), two functionally unknown genes belong to the ALOG 168 (Arabidopsis LSH1 and Oryza G1) transcription factor family (Fig. 3a). Intriguingly, the 169 loss-of-function mutations in ZmALOG1 and 2 caused enhanced tillering, similar as that in $170 t b 1$, gtl, and trul mutants (Fig. 3b; Supplementary Fig. 12a). The interactions between 171 ZmALOG1 and 2 and TRU1 were confirmed by Y2H assays (Fig. 3c). Moreover, 172 ZmALOG1 and 2 also interact with TB1 (Fig. 3c). These results demonstrate that the 173 interactions between known and unknown genes in subnetworks have biological meaning. 174 Therefore, our maize interactome can be used to reliably predict the functions of genes of 175 interest, as well as their putative interaction pathways, shedding light on the regulatory 176 mechanisms of known and unknown genes.

The interactome map contains functional modules with coherent sets of molecular 178 interactions for genes of similar genetic functions. CUP-SHAPED COTYLEDON (CUC) 179 genes establish boundaries between organs in many plant species ${ }^{19}$. However, the roles of 180 CUC genes in maize have not yet been well recognized. To test the suitability of the maize 181 interactome for discovering the functions of genes of interest, we searched the interactome 
182 modules and observed that the maize CUC genes ( $Z m C U C 3$ and its two paralogs ZmNAM1, 183 and ZmNAM2) interacted with TASSEL SHEATH1 (TSH1), BARREN STALK1 (BA1), and 184 PIN-FORMED1(ZmPIN1) (Fig. 3d); these genes (or their homologues) function in lateral 185 organ formation and development in maize or other species ${ }^{20-26}$. Thus, we reasoned that 186 maize CUC homologues might play similar roles in lateral organ development. Indeed, 187 loss-of-function zmnam1 and zmnam2 single and double mutants generated by CRISPR 188 technology exhibited suppressed lateral bud formation (Fig. 3e; Supplementary Fig. 12b), 189 confirming that these genes play conserved roles in lateral organ formation by establishing 190 boundaries, which are required for proper lateral meristem initiation. The interactions 191 between $\mathrm{ZmCUCs}$ and other genes, including BA1, TSH1 and unknown genes bHLH168 192 and MADS838 in the subnetwork are candidates for further research.

The interactome map can illustrate the molecular interactions for genes, which were 194 cloned separately for complex traits. Maize kernel size and weight are the most important 195 components of maize yield and have been intensively investigated. A total of 63 well196 known kernel mutant genes have been functionally cloned (Supplementary Table 6). We 197 identified 55 out of 63 genes in the interactome and divided them into two groups (40 for 198 constructing kernel-related subnetworks and 15 for validating the robustness of the kernel199 related regulatory network). The subnetworks of 40 randomly selected kernel genes (1000 200 simulations) successfully predicted up to $40 \%$ of the validated kernel genes, a value 201 significantly higher $(P=1 \mathrm{E}-6)$ than that of randomly selected functionally unrelated genes 202 (Supplementary Fig. 13a). Notably, all 55 kernel genes could be assembled into a linked 203 subnetwork in the maize interactome (Fig. 3f), which was further clustered into eight 204 modules. The genes in six modules were significantly enriched in the functional groups of 
205 embryo development, starch metabolic process, sugar mediated signalling pathway, and 206 metabolic process. Among these, the mutation of an unreported gene Zm00001d003047, 207 encoding a Pentatricopeptide Repeat-containing (PPR) protein, led to shrunken kernels 208 (Fig. 3f-3h; Supplementary Fig. 13b). Using the maize interactome, we also successfully 209 reconstructed regulatory networks involved in starch synthesis pathway ${ }^{27-30}$ and other 210 established pathways (Supplementary Fig. 14; Supplementary Table 7-8). Thus, the maize 211 interactome is a powerful tool for exploring and assembling gene regulatory networks 212 underlying complex traits, which could help breeders to optimize trait improvement. 

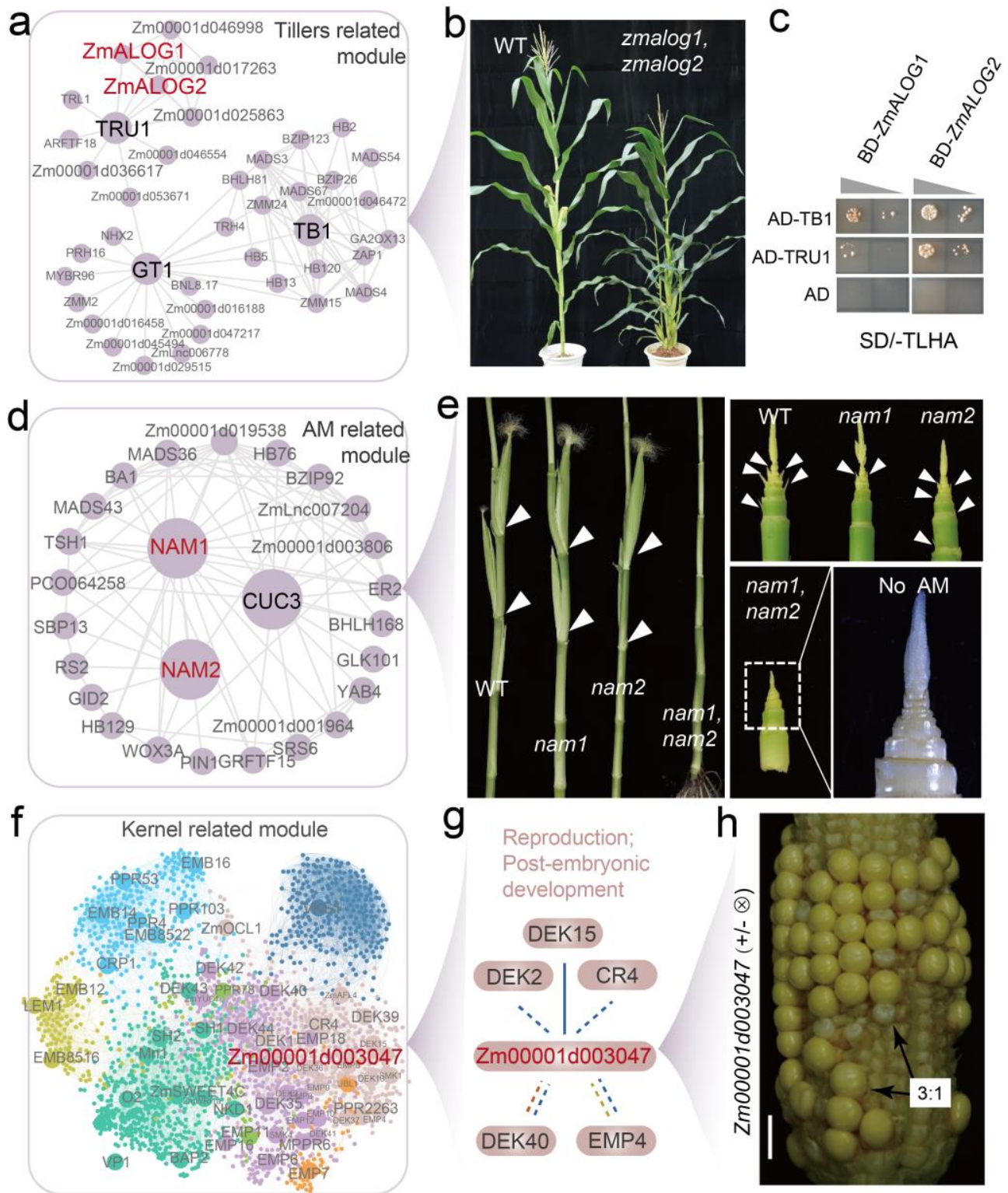

Figure 3. The interactome can predict gene function and re-construct regulatory networks underlying complex

traits. a, A network showing that the well-known tillering-related genes $t b 1$, trul, and gtl are connected to two novel genes (ZmALOG1 and ZmALOG2). b, Knockout mutants of zmalog1 and zmalog2 show altered tillering phenotypes. c, Protein interactions between ZmALOG1 and 2 and TRU1, TB1 were confirmed by Yeast-2-Hybrid assays. d, A network showing that $C U C 3$, a gene known to function in lateral tissue development, is connected to two novel genes, $N A M 1$ and NAM2. e, CRISPR/Cas9 knockout mutants of $N A M 1$ and $N A M 2$ show suppressed development of lateral meristem. f, Construction of regulatory networks to uncover the molecular mechanism conferring kernel development. The nodes contain genes with previously reported functions in kernel development. Different colours represent different modules associated with kernel variation. g, A gene of unknown function (Zm00001d003047) was identified in the newly constructed regulatory network based on the interactome of well-known kernel genes. h, The shrunken kernel phenotype 


\section{Systematically deciphering complex traits: flowering time as a}

\section{8 proof of concept}

Flowering time (FT) is an important agronomic trait involved in the adaptation of

230 maize to a wide range of climates worldwide ${ }^{31-33}$. Although many key FT genes in maize

231 have been cloned (Supplementary Table 9), the genetic control and molecular mechanisms

232 underlying this trait remain elusive. To dissect the potential regulatory networks, we

233 constructed a neutral prediction model based on the interactome map and machine learning

234 method using known FT genes (reported FT genes in maize and maize homologs to FT

235 genes cloned in other species) as the training dataset. The model showed high prediction

236 accuracy, and different layers of the interactome had variable prediction power, with AUC

237 values ranging from 0.68 to 0.89 (Fig. 4a). Notably, the integrative interactome has the

238 highest AUC value (up to 0.9). Totally, 3,553 genes were predicted to be associated with

239 FT at different confidence levels (Fig 4b; Supplementary Table 10), suggesting that an

240 ultracomplex molecular mechanism underlies FT in maize. We predicted the shortest

241 distance (SD) to known validated FT genes between all predicted FT genes and randomly

242 selected genes, and found that the predicted FT genes had significantly lower SD values

243 with validated FT genes compared to randomly selected genes (Fig. 4b). Using more

244 restrictive filtering via empirical cut-off of SD distribution, we obtained 1,843 high-

245 confidence FT genes (Fig. 4b; Supplementary Table 10). Compared with random genes,

246 the predicted FT genes are significantly enriched in both the Arabidopsis homology dataset 
247 (http://www.phytosystems.ulg.ac.be/florid/) and SNP-GWAS loci ${ }^{34}$ that are associated 248 with flowering time (Supplementary Fig. 15).

249 To assess the precision of our predictions, we chose 48 predicted genes and 10 250 training FT genes and successfully knocked out their functions by generating either 251 truncated protein or shifted reading frames for functional validation by CRISPR knockout 252 or EMS mutagenesis. In total, 30 genes (27 newly validated in maize and 3 reported FT 253 maize genes) were confirmed to be associated with FT variation (Fig.4c-4h; Supplementary 254 Table 11; Supplementary Fig. 16-45). Mutations in 9 of the 10 known FT genes led to 255 significant deviations in FT compared to the wild type in at least one growing location, 256 while knockout mutations in $43.75 \%$ (21/48) of the predicted FT genes led to significant 257 FT variation (Fig. 4c; Supplementary Table 11; Supplementary Fig. 16-45). For example, 258 loss-of-function mutation in the predicted gene Zm00001d06236, which encodes an MYB 259 transcription factor, could result in postponed flowering time in three environments (Fig. 260 4d-4f; Supplementary Fig. 16). Field tests on the other loss-of-function mutants showed 261 significant FT variations as expected (Fig. 4g; Supplementary Fig. 17-45).

262 Furthermore, based on a previous gene regulatory model ${ }^{35}$, our interaction network, 263 and the 30 validated FT genes, eight molecular pathways were found to be associated with 264 FT variation in maize: the light transduction pathway, circadian clock pathway, 265 photoperiod pathway, autonomous pathway, gibberellin (GA) pathway, a pathway for the 266 maintenance of inflorescence meristem identity (Integrator), the floral transition pathway, 267 and a newly uncovered pathway associated with histone modification (Fig. 4h; 268 Supplementary Table 12). These results indicate that machine learning mining on our 
269 interactome map is suitable for the large-scale identification of functional genes underlying 270 complex traits.

The addition of the 30 validated genes to molecular pathways involving FT in maize

272 further our understanding of FT variation (Fig. 4h). For example, Six genes were placed 273 into the light transduction pathway: Zm00001d024126 (Supplementary Fig. 17), 274 Zm00001d013402 (Supplementary Fig. 18), ZmPHYB1/236 (Supplementary Fig. 19), 275 Zm00001d033799 (Supplementary Fig. 20), ZmPHYC1/2 ${ }^{37}$ (Supplementary Fig. 21), 276 Zm00001d045944 (ZmCRY2, CRY2 homologue $\left.{ }^{38}\right)($ Supplementary Fig. 22). These genes 277 encode phytochrome $\mathrm{A} / \mathrm{B} / \mathrm{C}$ and photoreceptor cryptochrome proteins. Seven genes were 278 added into the circadian clock-related pathway: Zm00001d006236 (ZmMYB31) 279 (Supplementary Fig. 16), Zm00001d021291 (ZmPRR95a, circadian clock component 280 PRR9 homologue ${ }^{39}$ ) (Supplementary Fig. 23), Zm00001d006212 (ZmPRR95b, circadian 281 clock component PRR9 homologue ${ }^{39}$ ) (Supplementary Fig. 24), Zm00001d017241 282 (ZmTOC1b, TOC1 homologue $\left.{ }^{40}\right)($ Supplementary Fig. 25), Zm00001d049543 (ZmCCA1, 283 LHY and CCA1 homologue ${ }^{41}$ ) (Supplementary Fig. 26), Zm00001d034313 (Supplementary 284 Fig. 27), and Zm00001d006918 (Supplementary Fig. 28).

Notably, a newly identified FT pathway was uncovered in our model that includes 286 five newly validated genes (Fig. 4h): Zm00001d010625 (Supplementary Fig. 29, 287 Zm00001d011748 (Supplementary Fig. 30), Zm00001d017193 (Supplementary Fig. 31), 288 Zm00001d032894 (Supplementary Fig. 32), and Zm00001d006918 (Supplementary Fig. 289 33). Homologues of these newly discovered genes in Arabidopsis regulate the vernalization 290 response gene $F L C^{44}$ via epigenetic modification (H4K5ace ${ }^{45}$; antisense RNA synthesis ${ }^{46}$ ) 291 and nuclear complex formation ${ }^{47}$. Although vernalization does not occur in maize, maize 
292 genes whose homologues in Arabidopsis function in vernalization remain functional in 293 maize FT pathways. Genes in this pathway were enriched in the GO terms gene silencing 294 and histone modification (Supplementary Table 13). The unexpected discovery of a core 295 gene of this newly identified FT pathway in maize lays the foundation for studying the 296 relationship between FT and vernalization in domestication in the future ${ }^{13,32}$.

297 Since we systematically uncovered and validated a series of molecular pathways 298 underlying FT in maize, we asked whether genes from these pathways contribute in 299 consistent direction to FT variation. Loss-of-function mutants of FT genes grown in the 300 same environment (\{masked because of the Double-blind peer review.\}) exhibited a 301 wide range of significant FT variations (Fig. 4i; Supplementary Table 11). Interestingly, 302 loss-of-function mutations of genes from the same pathway had inconsistent genetic effects 303 on FT variation (Fig. 4i).

304 Flowering time is a key adaptive trait that has helped maize spread to a wide range of 305 environments worldwide. Many FT genes were selected during maize domestication and 306 improvement ${ }^{14}$. We asked whether specific FT pathways were preferentially subjected to 307 selection during maize domestication and improvement. Enrichment analysis of FT 308 pathway genes in genomic regions targeted for domestication and improvement ${ }^{14}$ 309 demonstrated that the floral transition pathway was significantly more likely to be subject 310 to selection during maize domestication than the other pathways (Supplementary Fig. 46). 311 Moreover, the GA pathway was significantly more likely to be selected during maize 312 improvement (Supplementary Fig. 46). Most importantly, genes with relatively high 313 genetic effects in the circadian clock and light transduction pathways have not been subject 

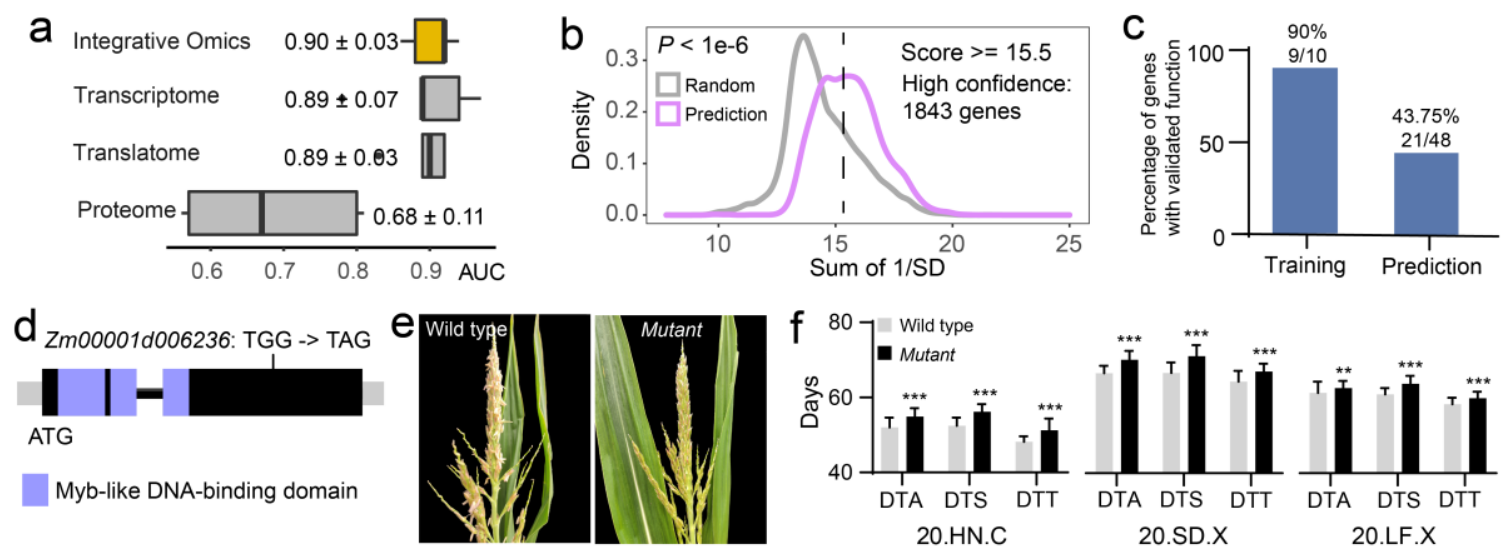

g

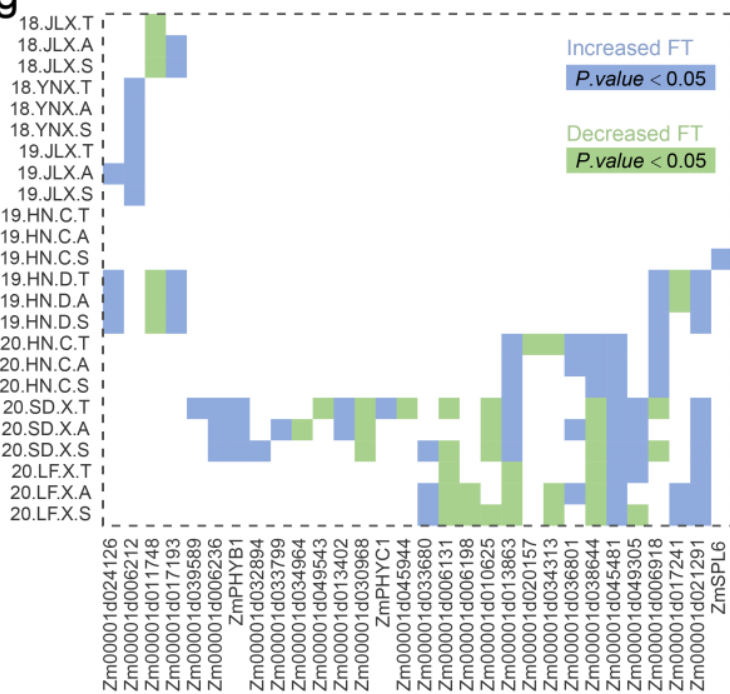

$\mathrm{h}$

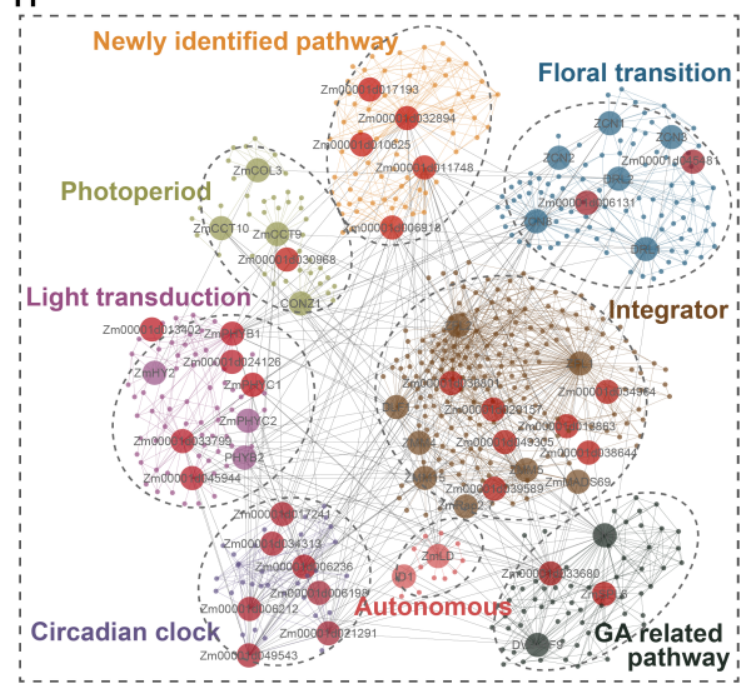

i

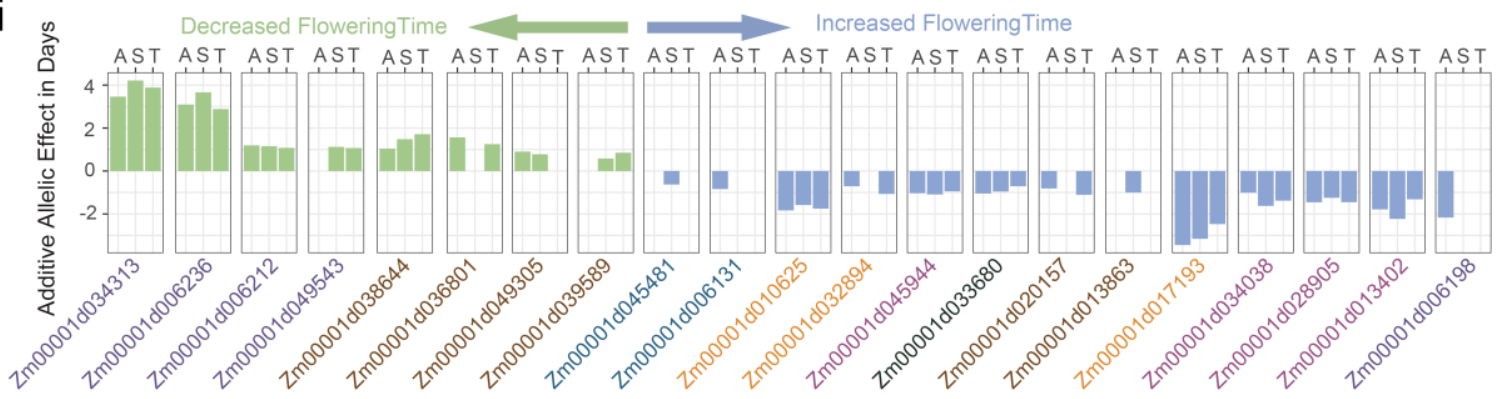

Figure 4. The interactome facilitates systematic dissection of the molecular mechanisms underlying flowering time in maize. a, Distribution of the AUC (Area Under Curve) values of different layers of the interactome for machine learning to predict flowering time (FT)-related genes. b, Distribution of SD values of predicted FT genes and randomly selected genes. SD represents the shortest distance to well-known FT genes. c, The percentage of genes with validated functions in loss-of-function mutants. d-f, Validation of the function of Zm00001d006236. d, An early stop codon 
mutation occurred in the last exon of Zm00001d006236. e. Zm00001d006236 mutants show delayed flowering time

323 compared to the wild type. f, Statistical comparison of FT variation between the wild type and Zm00001d006236 mutants

324 show that the mutant has significantly delayed FT compared to wild-type plants. **, and *** indicate P-values $<0.01$,

325 and 0.001 by Student's T-test, respectively. g, The 30 FT genes were shown to be significantly associated with FT

326 variation in multiple years or locations. For each gene, at least five individual plants per genotype have been used for the

327 phenotypic variation test. The label in y-axis shows year.location.season.trait. \{masked because of the

328 Double-blind peer review.\} C: spring; X: summer; D: winter; T: DTT (days to tasselling); A: DTA (days to

329 anthesis), S: DTS (days to silking). h, All validated FT genes (including well-known genes and newly validated genes in

330 this study) and their interaction genes could be classified into eight pathways. The 30 validated genes were highlighted

331 in red. i, Genetic effects of 21 FT genes in the same growing location. Different colours of gene ID on the x-axis represent

332 different pathways in Fig. 4h.

\section{Beyond the first interactome of maize}

The first interactome of maize uncovered more than 9 million interactions spanning

336 all levels of genetic information flow across the entire maize lifecycle. It is the largest

337 molecular functional network generated to date; however, it is still not saturated, especially

338 at the proteome level. To examine binary protein-protein interactions, we only screened

$33910,049 \mathrm{BD}$ bait genes against 24,586 AD prey genes, representing one-quarter of all

340 possible PPIs. Furthermore, the current interactome only describes intra-omic interactions

341 and not inter-omic interactions such as protein-DNA interactions, and protein-RNA

342 interactions. These are interesting questions for future research. 


\section{Conclusions}

345 Genetic information is derived from the genome and transferred to the 346 transcriptome, translatome, and finally the proteome. This process is regulated at multiple

347 levels simultaneously ${ }^{1}$. Dissecting these regulatory networks at multi-omics levels is a 348 highly efficient way to explore the functional genomics and life systems of an organism, 349 which are highly dynamic and complex ${ }^{1}$. We assembled a comprehensive interactome, 350 which contains genomic, transcriptomic, translatomic, and proteomic interactions. Our 351 data demonstrate that functional divergence exists at different layers and leads to gradually 352 increasing functional differentiation. Compared to the existing regulatory networks in 353 plants ${ }^{5,7}$, the current version of the maize interactome involves all layers of genetic 354 information flow, representing the largest interactome in plants generated to date. We 355 showed that the interactome facilitates the identification of the functions of key genes and 356 the systematic elucidation of the molecular mechanisms underlying phenotypic variations.

357 Thus, the maize interactome introduced here paves the way for systematically dissecting 358 the molecular mechanisms underlying agronomic traits, thereby revolutionizing genetic 359 research. It allows the simultaneous identification of hundreds of thousands of genes 360 involved in a trait and the construction of their interaction network. Coupled with genome 361 editing technologies, the interactome can guide systematic engineering of the agronomic 362 ally important trait in the near future. 
363 Acknowledgements: This research was supported by \{masked because of the Double-

364 blind peer review.\}.

365 Competing interests

366 The authors declare no competing interests. 


\section{References}

1. Trewavas, A. A Brief History of Systems Biology: "Every object that biology studies is a system of systems." Francois Jacob (1974). Plant Cell 18, 2420-2430 (2006).

2. Wallace, J. G., Larsson, S. J. \& Buckler, E. S. Entering the second century of maize quantitative genetics. Heredity 112, 30-38 (2014).

3. Ma'ayan, A. Complex systems biology. J. R. Soc. Interface 14, 20170391 (2017).

4. Dixon, S. J., Costanzo, M., Baryshnikova, A., Andrews, B. \& Boone, C. Systematic Mapping of Genetic Interaction Networks. Annu. Rev. Genet. 43, 601-625 (2009).

5. Consortium, A. I. M. Evidence for Network Evolution in an Arabidopsis Interactome Map. Science 333, 601-607 (2011).

6. Mergner, J. et al. Mass-spectrometry-based draft of the Arabidopsis proteome. Nature 579, 409414 (2020).

7. Walley, J. W. et al. Integration of omic networks in a developmental atlas of maize. Science 353, 814-818 (2016).

8. Yang, F. et al. Development and application of a recombination-based library versus library high- throughput yeast two-hybrid (RLL-Y2H) screening system. Nucleic Acids Res. 46, e17e17 (2018).

9. Peng, Y. et al. Chromatin interaction maps reveal genetic regulation for quantitative traits in maize. Nat. Commun. 10, 1-11 (2019).

10. Ingolia, N. T., Ghaemmaghami, S., Newman, J. R. S. \& Weissman, J. S. Genome-Wide Analysis in Vivo of Translation with Nucleotide Resolution Using Ribosome Profiling. Science 324, 218-223 (2009).

11. Wang, H., Studer, A. J., Zhao, Q., Meeley, R. \& Doebley, J. F. Evidence That the Origin of Naked Kernels During Maize Domestication Was Caused by a Single Amino Acid Substitution in tgal. Genetics 200, 965-974 (2015).

12. Schnable, J. C., Springer, N. M. \& Freeling, M. Differentiation of the maize subgenomes by genome dominance and both ancient and ongoing gene loss. Proc. Natl. Acad. Sci. USA. 108, 4069-4074 (2011).

13. Li, L. et al. Co-expression network analysis of duplicate genes in maize (Zea mays L.) reveals no subgenome bias. BMC Genomics 17, 875 (2016).

14. Hufford, M. B. et al. Comparative population genomics of maize domestication and improvement. Nature Genet. 44, 808-811 (2012).

15. Ecker, J. R. et al. ENCODE explained. Nature 489, 52-54 (2012). 
16. Doebley, J., Stec, A. \& Hubbard, L. The evolution of apical dominance in maize. Nature 386, 485-488 (1997).

17. Whipple, C. J. et al. grassy tillers 1 promotes apical dominance in maize and responds to shade signals in the grasses. Proc. Natl. Acad. Sci. USA. 108, 13375-13376 (2011).

18. Dong, Z. et al. Ideal crop plant architecture is mediated by tassels replace upper ears1, a BTB/POZ ankyrin repeat gene directly targeted by TEOSINTE BRANCHED1. Proc. Natl. Acad. Sci. USA. 114, E8656-E8664 (2017).

19. Hake, S. Identification of cup-shaped cotyledon: New Ways to Think about Organ Initiation. Plant Cell 31, 1202-1203 (2019).

20. Gallavotti, A., Yang, Y., Schmidt, R. J. \& Jackson, D. The Relationship between Auxin Transport and Maize Branching. Plant Physiol. 147, 1913-1923 (2008).

21. Yao, H. et al. The barren stalk2 Gene Is Required for Axillary Meristem Development in Maize. Mol. Plant. 12, 374-389 (2019).

22. Skirpan, A., Wu, X. \& McSteen, P. Genetic and physical interaction suggest that BARREN STALK1 is a target of BARREN INFLORESCENCE2 in maize inflorescence development. Plant J. 55, 787-797 (2008).

23. Whipple, C. J. et al. A Conserved Mechanism of Bract Suppression in the Grass Family. Plant Cell 22, 565-578 (2010).

24. Gälweiler, L. et al. Regulation of Polar Auxin Transport by AtPIN1 in Arabidopsis Vascular Tissue. Science 282, 2226-2230 (1998).

25. Wang, Q., Kohlen, W., Rossmann, S., Vernoux, T. \& Theres, K. Auxin Depletion from the Leaf Axil Conditions Competence for Axillary Meristem Formation in Arabidopsis and Tomato. Plant Cell 26, 2068-2079 (2014).

26. Gallavotti, A. et al. The role of barren stalk1 in the architecture of maize. Nature 432, 630-635 (2004).

27. Zhang, Z., Zheng, X., Yang, J., Messing, J. \& Wu, Y. Maize endosperm-specific transcription factors $\mathrm{O} 2$ and PBF network the regulation of protein and starch synthesis. Proc. Natl. Acad. Sci. USA. 113, 10842-10847 (2016).

28. Wang, W. et al. The Zea mays mutants opaque2 and opaque16 disclose lysine change in waxy maize as revealed by RNA-Seq. Sci Rep 9, 1-12 (2019).

29. Feng, F. et al. OPAQUE11 Is a Central Hub of the Regulatory Network for Maize Endosperm Development and Nutrient Metabolism. Plant Cell 30, 375-396 (2018).

30. Mach, J. Clarifying the Opaque: Identification of Direct Targets of Maize Opaque2. Plant Cell 27, 484-484 (2015). 
31. Kuleshov, N. N. World's diversity of phenotypes of maize. Journal of the American Society of Agronomy (1933).

32. Buckler, E. S. et al. The Genetic Architecture of Maize Flowering Time. Science 325, 714-718 (2009).

33. Heerwaarden, J. van, Hufford, M. B. \& Ross-Ibarra, J. Historical genomics of North American maize. Proc. Natl. Acad. Sci. USA. 109, 12420-12425 (2012).

34. Liu, H.-J. et al. CUBIC: an atlas of genetic architecture promises directed maize improvement. Genome Biol. 21, 20 (2020).

35. Dong, Z. et al. A Gene Regulatory Network Model for Floral Transition of the Shoot Apex in Maize and Its Dynamic Modeling. PLoS One 7, e43450 (2012).

36. Sheehan, M. J., Kennedy, L. M., Costich, D. E. \& Brutnell, T. P. Subfunctionalization of PhyB1 and $P h y B 2$ in the control of seedling and mature plant traits in maize. Plant J. 49, 338-353 (2007).

37. Li, Q. et al. CRISPR/Cas9-mediated knockout and overexpression studies reveal a role of maize phytochrome C in regulating flowering time and plant height. Plant Biotechnol. J. (2020).

38. Guo, H., Yang, H., Mockler, T. C. \& Lin, C. Regulation of Flowering Time by Arabidopsis Photoreceptors. Science 279, 1360-1363 (1998).

39. Matsushika, A., Imamura, A., Yamashino, T. \& Mizuno, T. Aberrant Expression of the LightInducible and Circadian-Regulated APRR 9 Gene Belonging to the Circadian-Associated APRR1/TOC1 Quintet Results in the Phenotype of Early Flowering in Arabidopsis thaliana. Plant Cell Physiol. 43, 833-843 (2002).

40. Somers, D. E., Webb, A. A., Pearson, M. \& Kay, S. A. The short-period mutant, toc1-1, alters circadian clock regulation of multiple outputs throughout development in Arabidopsis thaliana. Development 125, 485-494 (1998).

41. Mizoguchi, T. et al. LHY and CCA1 Are Partially Redundant Genes Required to Maintain Circadian Rhythms in Arabidopsis. Dev. Cell 2, 629-641 (2002).

42. Zhang, Y., Cao, G., Qu, L.-J. \& Gu, H. Characterization of Arabidopsis MYB transcription factor gene AtMYB17 and its possible regulation by LEAFY and AGL15. J. Genet. Genomics 36, 99-107 (2009).

43. Liu, H. et al. High-Throughput CRISPR/Cas9 Mutagenesis Streamlines Trait Gene Identification in Maize. Plant Cell (2020) doi:10.1105/tpc.19.00934.

44. Michaels, S. D. \& Amasino, R. M. FLOWERING LOCUS C Encodes a Novel MADS Domain Protein That Acts as a Repressor of Flowering. Plant Cell 11, 949-956 (1999). 
45. Xiao, J. et al. Requirement of histone acetyltransferases HAM1 and HAM2 for epigenetic modification of FLC in regulating flowering in Arabidopsis. J. Plant Physiol. 170, 444-451 (2013).

46. Shin, J.-H. \& Chekanova, J. A. Arabidopsis RRP6L1 and RRP6L2 Function in FLOWERING LOCUS C Silencing via Regulation of Antisense RNA Synthesis. PLoS Genet. 10, e1004612 (2014).

47. Gu, X. et al. Arabidopsis FLC clade members form flowering-repressor complexes coordinating responses to endogenous and environmental cues. Nat. Commun. 4, 1947 (2013). 


\section{Methods}

\section{$486 \quad$ Plant material}

Twenty-six tissues or stages (Supplementary Table 1) were sampled from B73

488 maize plants in a greenhouse. The plants were cultivated under a 12-h light/12-h dark cycle

489 at $25{ }^{\circ} \mathrm{C}$. Three replicates were prepared per tissue. RNA was extracted from 5-10

490 individual plants per replicate. Equal amounts of the three replicates were used for 491 sequencing.

\section{RNA sequencing platform}

Total RNA was isolated from the 26 tissues using a Direct-zol RNA Microprep kit

494 (Zymo Research) following the manufacturer's guidelines. Total RNA from each tissue 495 was reverse-transcribed to cDNA, which was used as a template to prepare the mRNA-seq, 496 sRNA-seq, and circRNA-seq libraries. The 26 RNA-seq libraries were prepared using 497 Ribo-Zero rRNA Removal kits and TruSeq Stranded Total RNA Library Prep Plant 498 (Illumina). The libraries were sequenced on the Illumina X-ten platform at the \{masked 499 because of the Double-blind peer review.\}. The 26 sRNA-seq libraries were prepared 500 using an MGIEasy Small RNA Library Prep Kit (BGI) and sequenced on a BGISEQ-500 501 sequencer at \{masked because of the Double-blind peer review.\}. The 26 circRNA-seq 502 libraries were prepared using Ribo-Zero rRNA Removal kits and a TruSeq RNA Library 503 Prep kit (Illumina). The circRNA libraries were sequenced on an X-ten platform at 504 \{masked because of the Double-blind peer review.\}.

\section{RNA-seq analysis}


BGI used SOAPnuke1.6.5 $5^{48}$ to process the raw RNA-seq and circRNA-Seq data 508 with the parameters "-n 0.01 -1 20 -q 0.3 -A 0.25" and used SOAPnuke1.6.5 ${ }^{48}$ filters RNA 509 to process the raw sRNA-Seq data with the parameters “-p 1 -z 18 -s -Q 2 --sanger”.

510

512 reference genome using the default parameters of HISAT and StringTie ${ }^{49-51}$. For each

513 tissue, a gtf file was generated and merged with an integrative annotation gtf file using 514 StringTie merge ${ }^{50}$. TPM (Transcripts Per Million reads), a standardized measure of gene 515 expression, was calculated with a 0.03 mismatch rate using RSEM 1.3.1 ${ }^{52}$.

\section{Identification and quantification of LncRNAs}

The improved version of LncRNA_Finder ${ }^{53}$ was used to identify lncRNAs from 518 total RNAs. Transcripts with more than 300 nucleotides were removed, as $>95 \%$ of the 519 proteins contained more than 100 amino acids ${ }^{54}$. The remaining transcripts were assessed 520 by $\mathrm{CPC} 2^{55}$, which rapidly and accurately assesses the coding ability of RNAs to 521 discriminate and remove potential protein-coding transcripts. To remove housekeeping 522 RNAs, including tRNAs (transfer RNAs) (http://gtrnadb.ucsc.edu/), rRNAs (ribosomal 523 RNAs) (http://maize.jcvi.org/), snRNAs (small nuclear RNAs), and snoRNAs (small 524 nucleolar RNAs) (http://noncode.org/), the remaining transcripts were compared to these 525 housekeeping RNA databases. The remaining transcripts were lncRNAs. RSEM ${ }^{52}$ software 526 was used to quantify lncRNA expression with a 0.03 mismatch rate. 
Mapsplice-v2.1.8 ${ }^{56}$ was used to identify fusion RNAs from total RNA-seq data

529 from each tissue. The following filtering steps were performed to obtain high-confidence

530 fusion RNAs. Firstly, PolyA sequences were removed using the following the criteria: the

531 proportion of As and Ts was $>40 \%$, and the proportion of As and Ts was $>80 \%$. Secondly,

532 to remove the fusion RNAs formed by homologous genes, the two parental genes

533 (overlapping sequences $>50 \mathrm{bp}$ ) that formed the fusion RNA were identified, and the

534 similarity between the parental genes was monitored. If the corresponding parental genes

535 could not be found (overlapping sequences $<50 \mathrm{bp}$ ), the sequences were extended $500 \mathrm{bp}$

536 up- and downstream of the splicing site, and the extended sequences were aligned the maize

537 AGPv4 genome ${ }^{57}$ to identify the parental genes. The remaining fusion RNAs were

538 compared to the genome by Blast-2.2.18 ${ }^{57}$, and the number of overlapping bases of the

539 splicing site was manually checked. Only fusion RNAs with $<7$ overlapping bases were

540 retained. Thirdly, since some fusion RNAs with high sequence similarity might be the same

541 fusion RNAs, Blast ${ }^{57}$ analysis was performed to divide the highly similar fusion RNAs into

542 groups, and the fusion RNAs with the highest number of reads in each group were retained.

543 Fourthly, to verify the identified fusion RNAs, their sequences were compared with their

544 original sequences using TopHat 2.1.1 ${ }^{58}$, and RNAs that match at least 6 bases and had a

545 read coverage of at least three at the junction site were retained. The normalised expression

546 levels of fusion RNAs were calculated as

$$
T P M_{f}=\frac{N i \times 10^{6}}{L i \times \operatorname{sum}(N i / L i+\ldots+N m / L m)},
$$


548 where $N i$ is the number of reads mapping to gene $\mathrm{i}$, and $L i$ is the sum of exon length of

549 gene i.

550 CircRNA identification and quantification

551 To obtain clean reads from circRNA-seq, FastUniq ${ }^{59}$ was used to remove duplicates

552 introduced by PCR amplification during high-throughput sequencing. CIRI v2.0.5 ${ }^{60}$ was

553 used to identify circRNAs by aligning them to the maize AGPv4 reference genome ${ }^{61}$, and

554 CIRCexplorer2 v2.0.1 ${ }^{62}$ was used quantify the expression levels of the circRNAs. The

555 mean counts of junction reads per mapped billion reads (CPM) values were calculated as

556 the normalised expression level of each transcript. The circRNA CPM formula is as follows:

$557 \quad C P M_{c}=\frac{\text { Reads }_{j} \times 10^{6}}{\text { Reads }_{m}}$

558 , where Reads $\mathrm{j}$ is the number of junction reads and Reads $\mathrm{m}_{\mathrm{m}}$ is the number of mapped reads.

559 sRNA identification and quantification

560 Small RNAs were identified and quantified as described by Wang et al. ${ }^{63}$ with slight

561 modifications. After filtering out low-quality reads and eliminating those matching tRNAs,

562 rRNAs, snRNAs, and snoRNAs, the retained clean reads from 26 tissues or stages that

563 were 18-26 nt in size were mapped to the maize AGPv4 reference genome using Bowtie-

564 1.1.2 with the parameters unique location and no mismatch ${ }^{51,61,64,65}$. All samples were

565 merged and the read number calculated using Samtools- $1.5^{66}$, and only sRNAs with more

566 than $6 \times$ coverage were retained. Given that sRNAs are distributed in clusters throughout

567 the maize genome and that gap distance is defined as the distance between two 
568 neighbouring clusters, we compared the coverage of chromosomes between two 569 neighbouring sRNA clusters using different gap distances and selected a coverage of $6 \times$ 570 and a 300 bp gap distance as the thresholds for identifying sRNAs (Supplementary Fig.

571 1h,1i). For each sample, the number of sRNA reads in each sRNA cluster was calculated 572 and reconstructed as a matrix, with each row representing a sRNA cluster and each column 573 representing a sample. Read abundances were normalised based on library size by scaling 574 to TPM to allow for direct comparisons across libraries.

\section{Ribo-seq: preparation, sequencing, and analysis}

576 To extract polysomes, tissue $(5 \mathrm{~g})$ samples were pulverized and dissolved in 577 polysome extraction buffer (44 mM Tris- $\mathrm{HCl}, \mathrm{pH} 7.5,175 \mathrm{mM} \mathrm{KCl}, 13 \mathrm{mM} \mathrm{MgCl}, 1 \%$ 578 TritonX-100, $15 \mathrm{mM}$ 2-mercaptoethanol, $100 \mu \mathrm{g} / \mathrm{mL}$ cycloheximide, 10 units/mL DNase 579 I). The polysome pellets were treated with RNase I (10 units/ $\mu \mathrm{g}$ RNA) at room temperature 580 for $1 \mathrm{~h}$. After terminating the reaction using RNase Inhibitor (20 units/ $\mu \mathrm{L})$, the solution 581 was immediately transferred into a MicroSpinS-400 column to enrich the RNA-ribosome 582 complex (monosomes). Ribosome-protected fragments were extracted from the samples 583 using a miRNeasy RNA isolation kit (Qiagen) following the manufacturer's instructions. 584 After removing rRNAs, the remaining RNAs were used to construct libraries, which were 585 sequenced on the Illumina HiSeq 2500 platform.

After removing adaptors and quality control using FASTX_Toolkit-0.0.14 587 (http://hannonlab.cshl.edu/fastx_toolkit/index.html) fastx_clipper with the parameters “-1 $5885-\mathrm{c}-\mathrm{n}-\mathrm{v}-\mathrm{Q} 33$ ” and fastx_trimmer with the parameters “-f 1 -Q 33", the Ribo-Seq reads 589 were aligned to the rRNA reference sequence from NCBI using Bowtie-1.1.2 ${ }^{67}$ to filter out 
590 reads derived from rRNAs. The Ribo-Seq reads were mapped to the maize AGPv4 591 reference genome ${ }^{61}$. The expression levels were measured by TPM using RSEM (version $592 \quad 1.3 .0)^{52}$. Only reads that mapped to the coding sequence were used to calculate translational 593 abundance.

\section{Protein-protein interactions}

595

596

597

598

599

600

601

602

603

604

605

606

607

608

609

610

611

\section{PCR amplification}

Total RNA was extracted from 20 tissues separately using a Direct-zol RNA Miniprep kit (Zymo Research). The total RNA was divided into eight groups (Supplementary Table 3) based on tissue type. Equal amounts of RNA from each group were pooled. Approximately $2 \mu \mathrm{g}$ pooled RNA was used to generate first-strand cDNA using Clontech's SMART TM technology ${ }^{21}$ according to Clontech's Make Your Own "Mate \& Plate"TM" Library System User Manual (Protocol No. PT4085-1) with modified primers. These modified primers were homologous to modified pGADT7 (mAD) or modified pGBKT7 (mBD) vectors from a previously reported recombination-based library versus library high-throughput yeast two-hybrid (RLL-Y2H) screening system ${ }^{8}$ and included modified

SMART

III oligo

primers (CCATACGACGTACCAGATTACGCTCC/rG//rG//rG/ [rG represents a ribonucleotide] for the mAD vector and AAGCTGATCTCAGAGGAGGACCTGCC/rG//rG//rG/ for the $\begin{array}{lllll}\mathrm{mBD} & \text { vector) } & \text { and } & \text { modified }\end{array}$ (GGGCGTTGACACTAGTCTCGAGGATC(T) ${ }_{30} \mathrm{VN}, \mathrm{N}=\mathrm{A}, \mathrm{G}, \mathrm{C}$, or T; V=A, G, or C) for the mAD vector and ACCCGCACGCTAACTAGTGTCGAC(T) $)_{30} \mathrm{VN}$ for the $\mathrm{mBD}$ vector). The first-strand cDNA was amplified to obtain full-length double-stranded cDNA 
612 using a KAPA HiFi HotStart ReadyMix PCR kit with specific primers (Forward primers:

613 AGCGCCGCCATGGAGTACCCATACGACGTACCAGATTACGCT and

614 GCCATCATGGAGGAGCAGAAGCTGATCTCAGAGGAGGACCTG for the mAD and

$615 \mathrm{mBD} \quad$ vectors, respectively; $\quad$ Reverse

616 CCTTTGAGTTCTCTCAGTTGGGGGCGTTGACACTAGTCTCGA and

617 CCAAGGGCACGCCCTGGCACCCGCACGCTAACTAGTGTCGAC for the mAD and $618 \mathrm{mBD}$ vectors, respectively). Next, the full-length double-stranded cDNA was normalised 619 as described previously ${ }^{68}$, and $10 \mu \mathrm{g}$ of normalised cDNA was used for each yeast 620 transformation.

621 Yeast library construction

622 Modified $\mathrm{mAD}$ and $\mathrm{mBD}$ vectors were used to construct a maize cDNA yeast

623 library according to the Clontech $\mathrm{Y} 2 \mathrm{H}$ transformation method ${ }^{8}$. The $\mathrm{mAD}$ and $\mathrm{mBD}$ 624 vectors were digested with the restriction enzymes BamHI and NdeI. Each $10 \mu \mathrm{g}$ PCR625 amplified cDNA fragment was mixed with $5 \mu \mathrm{g}$ linearized mAD or mBD vector and used 626 to co-transform $6 \mathrm{ml} \mathrm{Y187}$ and $\mathrm{Y} 2 \mathrm{H}$ yeast-competent cells, respectively, with the 627 Yeastmaker Yeast Transformation System (Clontech) following the manufacturer's 628 protocol. The cells were spotted onto SD -Leu (mAD clones) or SD -Trp + X- $\alpha-\mathrm{Gal}(\mathrm{mBD}$ 629 clones) medium and grown for 3-4 days at $30^{\circ} \mathrm{C}$. All blue clones including self-activated 630 functional fusion genes in the BD library were removed.

$631 \quad$ Yeast mating

To identify positive yeast mating, $2 \mathrm{ml}$ of $\mathrm{Y} 2 \mathrm{H}$ and $\mathrm{Y} 187$ yeast libraries (at least 2 $633 \times 107$ cell each library) were combined in $50 \mathrm{ml} 2 \times$ YPDA liquid medium and incubated at 
$63430^{\circ} \mathrm{C}$ for $24 \mathrm{~h}$ at a shaking speed of $50 \mathrm{rpm}$. The yeast cells were collected and resuspended 635 in $6 \mathrm{ml} 0.9 \% \mathrm{NaCl}$, and $300 \mu \mathrm{L}$ aliquots were plated onto a $15 \mathrm{~cm}$-diameter cell-culture 636 dish containing SD/-Trp, -Leu, -His, -Ade selective solid medium and grown for 5-7 days

637 at $30^{\circ} \mathrm{C}$. Each library mating was repeated at least 10 times. A barcode sequence was added 638 to each repeat, and ten repeats were pooled to construct a sequencing library.

639 Yeast plasmid isolation and PPI sequencing

640 Following yeast mating, the clones on plates for each repeat were collected and the 641 fusion plasmids extracted from the yeast cells using a yeast plasmid kit (OMEGA). 642 Barcoded (Third-generation sequencing barcodes) vector primers (Supplementary Table 643 14) were used to amplify the plasmids for each repeat. This PCR products were further 644 purified using DNA-free beads to remove fragments shorter than $0.75 \mathrm{~kb}$.

\section{BiFC assays}

646 To validate the positive rate of the PPI data, 114 protein-protein interactions were 647 randomly chosen from three libraries and the corresponding cDNA fragments cloned in the 648 Pxy104 and Pxy106 vectors using a ClonExpress II One Step Cloning Kit (Vazyme, 649 Nanjing, China). These BiFC constructs were transformed into Agrobacterium tumefaciens 650 strain GV3101 and co-transfected into Nicotiana benthamiana leaf epidermal cells. The 651 YFP signals in infiltrated leaves were observed and photographed $48 \mathrm{~h}$ later under an SP8 652 confocal microscope (Leica) with $488 \mathrm{~nm}$ excitation and 515-555 nm emission filters.

\section{PPI analysis}


The libraries were sequenced on the PacBio platform, and CCS (Circular

655 Consensus Sequencing) reads were called using SMRTLink-5.1.0.26412

656 (https://www.pacb.com/support/software-downloads/). The following analysis was

657 performed to obtain PPIs: Firstly, reads in FASTA format were extracted from BAM files

658 using SAMtools-1.966. Next, because ten barcoded samples were combined to generate

659 each sequencing library, reads from each sample were divided based on their barcode

660 sequences using Cutadapt-1.9.1 ${ }^{69}$. Parameters -e and $-\mathrm{m}$ for running reads separation were

6610.3 and 200, respectively. The reads were aligned to the coding sequence data from the

662 maize AGPv4 genome to identified candidate PPI genes using BLAST+/2.7.1 ${ }^{57}$. Only PPI

663 genes with BLAST e-value $<1.0 \mathrm{e}^{-4}$ that matched to two genes were retained. Lastly, the

664 mAD-primer (CATACGACGTACCAGATTACG), mBD-primer

665 (CATCATGGAGGAGCAGAAGCT), and recombination sequences

666 (TAGCGTGCGGGTGCCAGGGCGTGCCCTTGAGTTCTCTCAGTTGGGGGCGTTG

667 AC) were aligned to the retained CCS reads using needle

668 (http://biopython.org/DIST/docs/tutorial/Tutorial.html\#htoc86). If a CCS contained

669 sequences from two different genes, and the primer sequence and the recombination

670 sequence were aligned consistently, the proteins encoded by the two genes were considered

671 to be interacting proteins.

672 The PPIs were divided into three confidence groups based on the probability of

673 self-activation. Low-confidence PPIs indicates interaction edges detected using offline data.

674 Because the mating of yeasts with empty vectors generated interaction signals (self675 activation), to remove edges caused by self-activation, all PPIs involving eight empty BD

676 libraries were removed to obtain the middle-confidence PPIs. To further remove false PPIs 
677 introduced by self-activation, PPIs identified using empty vectors were used as the positive

678 training data, true PPIs confirmed by BiFC were used as the negative training data, and the

679 frequencies of each gene in the $\mathrm{AD}$ and $\mathrm{BD}$ libraries were used as training features to

680 estimate the self-activating genes using the M5P machine learning classification algorithm

681 (https://cran.r-project.org/web/packages/RWeka/index.html). Edges for self-activating

682 genes in the BD library were removed, and the remaining PPIs were considered to be high-

683 confidence PPIs.

\section{Construction of co-expression networks}

An expression matrix of 179,309 components (25,319 genes, 5,469 lncRNAs, 45

686 fusion RNAs, 4,318 circRNAs, and 144,158 small RNAs) detected in more than 10 of 26

687 distinct tissues or stages were used to construct a transcriptome co-expression network. To

688 reduce the bias of Gini Correlation Coefficient (GCCs) caused by overexpressed transcripts,

689 TPM values were transformed by a logarithmic function. GCCs between all pairs of all

690 elements $(179,309 \times 179,309)$ were calculated using the adjacency matrix rsgcc_1.0.6 $6^{70}$

691 with "saveType="bigmatrix"", which is suitable for big data. Any edge with GCCs $<0.3$

692 was excluded. Next, Mutual Rank $\mathrm{a}_{\mathrm{a}, \mathrm{b}}$, i.e., the mutual rank between element a and element

$693 \mathrm{~b}$, was employed to assess the correlation of co-expression according to the formula ${ }^{71,72}$ :

$$
\text { Mutual } \operatorname{Rank}_{a, b}=\sqrt{\operatorname{rank}\left(G C C_{a}->G C C_{b}\right) \times \operatorname{rank}\left(G C C_{b}->G C C_{a}\right)}
$$

695 Mutual correlation was divided into three confidence levels based on the relationship

696 between gene number and MR (Supplementary Fig. 3). MR values at rapidly increasing

697 intervals, slowly increasing intervals, and intervals reaching a steady level were defined by 
698 dividing by the high-, middle-, and low confidence values, respectively (Supplementary 699 Fig. 3).

Finally, the Edge Weight (EW) between node a and node $\mathrm{b}$ was converted by $\mathrm{MR}_{\mathrm{a}, \mathrm{b}}$

701 values. Larger EW values indicate greater correlation according to the formula:

702

$$
\text { Edge Weight }_{a, b}=\mathrm{e}^{-\frac{\mathrm{MRa}, \mathrm{b}-\min (\mathrm{MRa}, \mathrm{b})}{\max (M R a, b)-\min (M R a, b)}}
$$

703 , where EW ranges from $\frac{1}{e}$ to 1 .

To obtain a network containing high-confidence small RNAs with potential 705 functional implications, co-expression networks were constructed using 25,300 genes, 706 5,469 lncRNAs, 14 fusion RNAs, 4,318 circRNAs, and 159 micro RNAs. High, middle, 707 and low-confidence edges were identified as described above. In addition, a co-expression 708 network only including 24,394 annotated genes was constructed named the Slim709 transcriptome network; high, middle, and low-confidence edges were identified in this 710 network as described above.

\section{Construction of an integrative omics database}

Four omic data sets, including ChIA-PET data from Peng et al. ${ }^{9}$, a co-expression

713 network constructed using RNA-Seq data from 26 tissues or stages, a co-expression

714 network constructed using Ribo-seq data from 20 tissues or stages, and PPIs from yeast-2-

715 hybrid screening were used to construct an integrative omic database including all possible

716 temporal and spatial interactions. An integrative $\mathrm{EW}\left(\mathrm{EW}_{\mathrm{i}}\right)$ was calculated as detailed

717 below. If an edge was present in one omic data set, the EW was raised to the fourth power

718 to obtain the $\mathrm{EW}_{\mathrm{i}}$. If an edge was present in two omic data sets, $\mathrm{EW}_{\mathrm{i}}$ was calculated by 
719 multiplying the square of the average $\mathrm{EW}$ by each $\mathrm{EW}$. If three edges were present, $\mathrm{EW}_{\mathrm{i}}$

720 was calculated by multiplying the average EW by each EW. If four edges were present,

$721 \mathrm{EW}_{\mathrm{i}}$ was calculated by multiplying each $\mathrm{EW}^{12}$. Each integrative omic edge included four

722 (genome, transcriptome, translatome, and proteome) corresponding omic edges. Because

723 each omic data point had three corresponding confidence levels, three $\mathrm{EW}_{\mathrm{i}}$ values

724 representing three confidence levels were ultimately obtained.

\section{Machine learning}

726 Thirty-nine FT genes (related to flowering time) from the literature were collected

727 as the positive training data, and 39 non-FT genes selected from the Classical Genes of the

728 MaizeGDB database (https://www.maizegdb.org/) were used as the negative training data

729 (Supplementary Table 9). Training data included the TPM of 26 tissues or stages in the

730 transcriptome, the TPM of 20 tissues or stages in the translatome, and the SD values of the

73178 genes in the proteome. A matrix was assembled as the input file, in which the first row

732 listed the genes to predict and the first column listed the 124 training features. The Scikit-

733 learn package (version 0.21.2; https://scikit-learn.org/stable/) was used to identify FT and

734 non-FT genes with default parameters. Three models of machine learning were used to

735 train and extract the data attributes: LogisticRegression, Support Vector Machine, and

736 adaboost (https://scikit-learn.org/stable/). Five-fold cross-validation was used to assess the

737 prediction accuracy, and ROC-AUC (Receiver-Operating Characteristic Area Under the

738 Curve), a standard performance evaluation criterion used for two-class pattern

739 classification problems, was used to compare different classification algorithms.

\section{Phenotypic verification}


743 Zm00001d021291 were generated using a high-throughput genome-editing system ${ }^{30}$. In

744 brief, line-specific sgRNAs (single guide RNAs) were designed based on the assembled 745 pseudo-genome of the receptor KN5585. A double sgRNAs pool (DSP) approach was used 746 for vector construction. The receptor KN5585 was transformed with the vectors, and the 747 targets of each T0 plant were examined by barcode-based sequencing. The genotype of 748 each gene-edited line was identified by PCR amplification and Sanger sequencing using 749 target-specific primers (Supplementary Table 15). The flowering times of the gene-edited 750 lines and the receptor KN5585 were investigated, including days to tasselling (DTT), days 751 to anthesis (DTA), and days to silking (DTS).

Positive transgenic lines of the four genes were evaluated for flowering time, 753 including Zm00001d006918, ZmSPL6 (Zm00001d042319), Zm00001d017241, and 754 Zm00001d021291. In the summer of 2018 in \{masked because of the Double-blind peer 755 review.\}, the homozygous transgene-positive lines of Zm00001d006918 (T2 generation) 756 and Zm00001d017241 (T1 generation) and their negative counterparts were evaluated for 757 flowering time. In the summer of 2018 in \{masked because of the Double-blind peer 758 review.\}, the homozygous transgene-positive lines of ZmPRR95a (T1 generation) and its 759 negative counterparts were evaluated for flowering time. In the summer of 2019 in 760 \{masked because of the Double-blind peer review.\}, the homozygous transgene-positive 761 lines of zmspl6 (T4 generation) and Zm00001d021291 (T4 generation) and their negative 762 counterparts were evaluated for flowering time. The homozygous transgene-positive lines 763 of Zm00001d006918 (T6 generation), zmspl6 (T5 generation), and Zm00001d017241 
764 (T5 generation) and their negative counterpart were evaluated for flowering time in the 765 Winter of 2019 in \{masked because of the Double-blind peer review.\}.

766 EMS mutants

767 To verify the functions of the candidate genes, 54 mutants with SNP mutations 768 leading to early translational stops, one mutant with an SNP mutation that changed the start 769 codon, and one mutant with an SNP mutation at the splice site from a maize EMS mutant 770 library $^{73}$ were obtained. The mutants were planted in \{masked because of the Double-

771 blind peer review.\}. DNA was extracted from mutant seedlings and used to genotype each 772 plant and to identify the homozygous mutant plants and their wild-type counterparts. DTT, 773 DTA, and DTS of the mutant and wild-type plants were evaluated, and the results were 774 subjected to one-tailed $t$ test to compare the significance of their differences.

\section{Other analysis}

776 Four attributes, including Degree, Shortest distance, Average distance, and 777 Transitivity were calculated for each network using the $\mathrm{R}$ package igraph-1.2.4.2 778 (https://igraph.org/r/). Gene ontology analysis was performed using AGRIGO version2 779 (http://systemsbiology.cau.edu.cn/agriGOv2/index.php). Interactions in the network were 780 clustered using the Markov Cluster Algorithm (https://github.com/JohannesBuchner/mcl). 781 Gephi (https://gephi.org/) was used for visualization and feature extraction of the co782 expression networks. 


\section{Supplementary References}

48. Cock, P. J. A., Fields, C. J., Goto, N., Heuer, M. L. \& Rice, P. M. The Sanger FASTQ file format for sequences with quality scores, and the Solexa/Illumina FASTQ variants. Nucleic Acids Res. 38, 1767-1771 (2010).

49. Kim, D., Langmead, B. \& Salzberg, S. L. HISAT: a fast spliced aligner with low memory requirements. Nat. Methods 12, 357-360 (2015).

50. Kovaka, S. et al. Transcriptome assembly from long-read RNA-seq alignments with StringTie2. Genome Biol. 20, 278 (2019).

51. Wang, B. et al. Unveiling the complexity of the maize transcriptome by single-molecule longread sequencing. Nat. Commun. 7, 1-13 (2016).

52. Li, B. \& Dewey, C. N. RSEM: accurate transcript quantification from RNA-Seq data with or without a reference genome. BMC Bioinformatics 12, 323 (2011).

53. Li, L. et al. Genome-wide discovery and characterization of maize long non-coding RNAs. Genome Biol. 15, R40 (2014).

54. Frith, M. C. et al. The Abundance of Short Proteins in the Mammalian Proteome. PLoS Genet. 2, e52 (2006).

55. Kang, Y.-J. et al. CPC2: a fast and accurate coding potential calculator based on sequence intrinsic features. Nucleic Acids Res. 45, W12-W16 (2017).

56. Wang, K. et al. MapSplice: Accurate mapping of RNA-seq reads for splice junction discovery. Nucleic Acids Res. 38, e178-e178 (2010).

57. Johnson, M. et al. NCBI BLAST: a better web interface. Nucleic Acids Res. 36, W5-W9 (2008).

58. Trapnell, C., Pachter, L. \& Salzberg, S. L. TopHat: discovering splice junctions with RNASeq. Bioinformatics 25, 1105-1111 (2009).

59. Xu, H. et al. FastUniq: A Fast De Novo Duplicates Removal Tool for Paired Short Reads. PLoS One 7, e52249 (2012).

60. Gao, Y., Wang, J. \& Zhao, F. CIRI: an efficient and unbiased algorithm for de novo circular RNA identification. Genome Biol. 16, 4 (2015).

61. Jiao, Y. et al. Improved maize reference genome with single-molecule technologies. Nature 546, 524-527 (2017).

62. Zhang, X.-O. et al. Diverse alternative back-splicing and alternative splicing landscape of circular RNAs. Genome Res. 26, 1277-1287 (2016). 
63. Wang, J., Yao, W., Zhu, D., Xie, W. \& Zhang, Q. Genetic basis of sRNA quantitative variation analyzed using an experimental population derived from an elite rice hybrid. eLife $\mathbf{4}$, e03913 (2015).

64. Langmead, B., Trapnell, C., Pop, M. \& Salzberg, S. L. Ultrafast and memory-efficient alignment of short DNA sequences to the human genome. Genome Biol. 10, R25 (2009).

65. Crisp, P. A. et al. Variation and Inheritance of Small RNAs in Maize Inbreds and F1 Hybrids. Plant Physiol. 182, 318-331 (2020).

66. Li, H. et al. The Sequence Alignment/Map format and SAMtools. Bioinformatics 25, 20782079 (2009).

67. Langmead, B. Aligning Short Sequencing Reads with Bowtie. Current Protocols in Bioinformatics 32, 11.7.1-11.7.14 (2010).

68. A. Bogdanova, E., A. Shagin, D. \& A. Lukyanov, S. Normalization of full-length enriched cDNA. Mol. Biosyst. 4, 205-212 (2008).

69. Martin, M. Cutadapt removes adapter sequences from high-throughput sequencing reads. EMBnet.journal 17, 10-12 (2011).

70. Ma, C. \& Wang, X. Application of the Gini Correlation Coefficient to Infer Regulatory Relationships in Transcriptome Analysis. Plant Physiol. 160, 192-203 (2012).

71. Obayashi, T. \& Kinoshita, K. Rank of Correlation Coefficient as a Comparable Measure for Biological Significance of Gene Coexpression. DNA Res. 16, 249-260 (2009).

72. Wisecaver, J. H. et al. A Global Coexpression Network Approach for Connecting Genes to Specialized Metabolic Pathways in Plants. Plant Cell 29, 944-959 (2017).

73. Lu, X. et al. Gene-Indexed Mutations in Maize. Mol. Plant. 11, 496-504 (2018).

74. Qiao, X. et al. Gene duplication and evolution in recurring polyploidization-diploidization cycles in plants. Genome Biol. 20, 38 (2019).

75. Freeling, M. Bias in Plant Gene Content Following Different Sorts of Duplication: Tandem, Whole-Genome, Segmental, or by Transposition. Annu. Rev. Plant Biol. 60, 433-453 (2009).

76. Shao, H., Wang, H. \& Tang, X. NAC transcription factors in plant multiple abiotic stress responses: progress and prospects. Front. Plant Sci. 6, (2015). 


\section{Supplementary}

\section{Supplementary Text}

\section{Regulatory divergence between duplicate genes}

Speciation and domestication were accompanied by whole-genome duplication

848 (WGD), tandem duplication (Tandem), proximal duplication (Proximal), transposed 849 duplication (Transposed) mediated by transpositions, and dispersed origination 850 (Dispersed) $)^{74,75}$. In our interactome, gene interaction modules contain genes with 851 functionally conserved roles, and Shortest Distance (SD)

852 (https://igraph.org/r/doc/distances.html), which represents the shortest path between a pair 853 of nodes, describes the functional divergence for any pair of duplicate genes. As expected, 854 all duplicates had significantly higher functional conservation and lower functional 855 divergence compared to randomly selected genes, supporting the validity of our first856 generation interactome of maize. When we compared duplicated genes, we observed 857 consistent tendencies for both functional conservation and divergence (Supplementary Fig. 858 8a-8d): the functions of Tandem and Proximal genes were more likely to be conserved, 859 whereas the functions of Transposed genes were least likely to be conserved 860 (Supplementary Fig. 8a-8d). WGD, Tandem, and Proximal genes had the lowest SD values 861 and the least functional divergence, while Transposed and Dispersed genes had the highest 862 SD values and the most functional divergence (Supplementary Fig. 8a-8d). To dissect the 863 regulatory divergence of the maize subgenomes, we compared the degree (number of 864 connections with its directly adjacent edges in the interactome) values of genes at different 865 omic layers. The genes were classified into four groups, Maize1 genes with retained 
866 Maize2 orthologs, Maize1 genes without retained Maize2 orthologs, Maize2 genes with 867 retained Maize1 orthologs, and Maize2 genes without retained Maize1 orthologs. The

868 degree variations of four different gene groups have been calculated and compared 869 (Supplementary Fig. 8g,8h). Additionally, the dominant subgenome bins were obtained

870 through Chi-square test on the degree to which the proportion of genes in the subgenome

871 (out of 100) in a sliding window is significantly higher than that of the corresponding

872 subgenome genes displayed based on the gene order along sorghum chromosomes

873 (Supplementary Fig. 9).

\section{Example of hub genes suffering from selection}

875 The transcriptome-level Maize2 hub gene ZmNAC75, encoding a NAC domain 876 transcription factor, interacted with four regulatory modules. Genes from three ZmNAC75877 regulated modules were significantly enriched in the gene ontology $(\mathrm{GO})$ terms stomatal 878 movement, ion transport, and ABA-activated pathway. Many stress-responsive NAC TFs 879 have been used to improve stress tolerance in $\operatorname{crops}^{76}$. The regulatory networks of $880 Z m N A C 75$ reveal the molecular basis of stress responses to some extent (Supplementary 881 Fig. 11a). Analysis of the nucleotide diversity of wild maize relatives, landraces, and 882 modern inbreds indicated that $Z m N A C 75$ has been a selection target during modern maize 883 improvement (Supplementary Fig. 11b). 

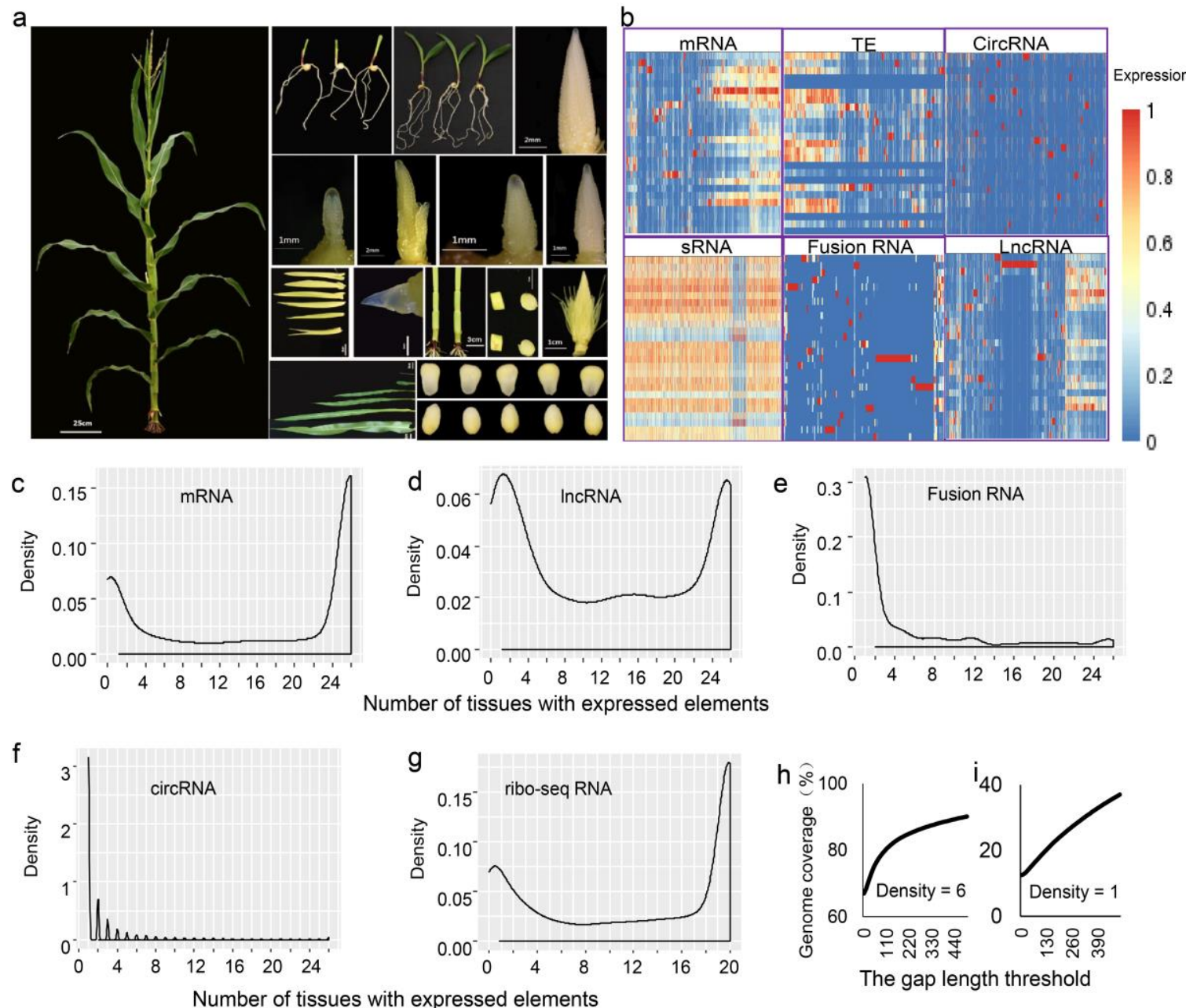

Supplementary Fig. 1. Expression-level variation across 26 different stages/tissues for all different types of transcripts. a. Sampling of 26 stages/tissues spanning the maize whole life period. b. Transcriptome landscape of all 26 different stages/tissues for all different types of transcript isoforms. c-f. Stage/tissue-specific expression of proteincoding, long noncoding, fusion, and circular transcripts. g. Stage/tissue-specific abundance of ribosome-imprinted transcripts. h. Genome coverage for small RNAs at different cutoffs. 


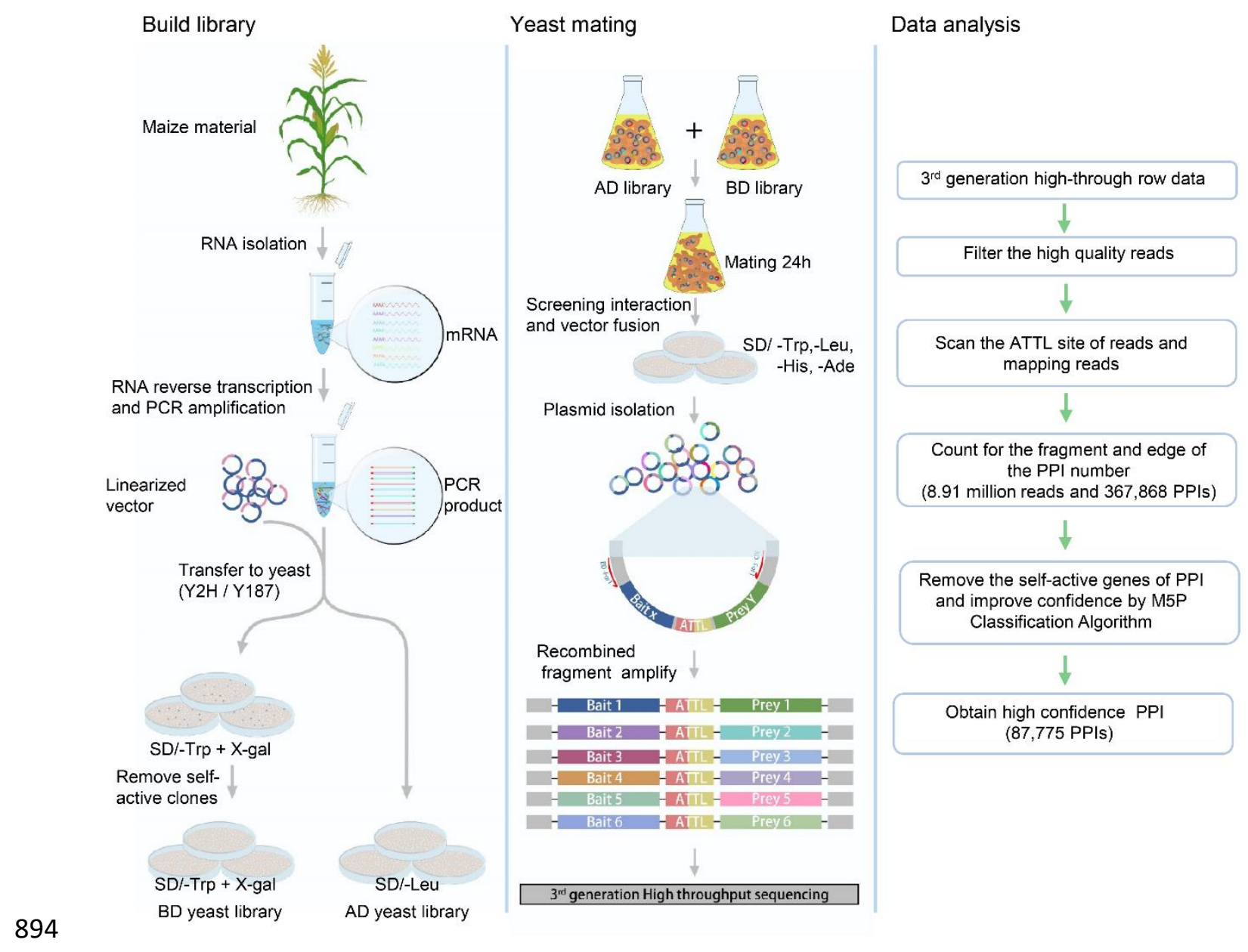

895 Supplementary Fig. 2. The pipeline of high-throughput yeast-2-hybrid for the 896 identification of protein-protein interactions. This pipeline consists of three major steps, 897 yeast cDNA library construct, yeast mating and data analysis. Detailed methods can be 898 found in the Method part.

899

900 

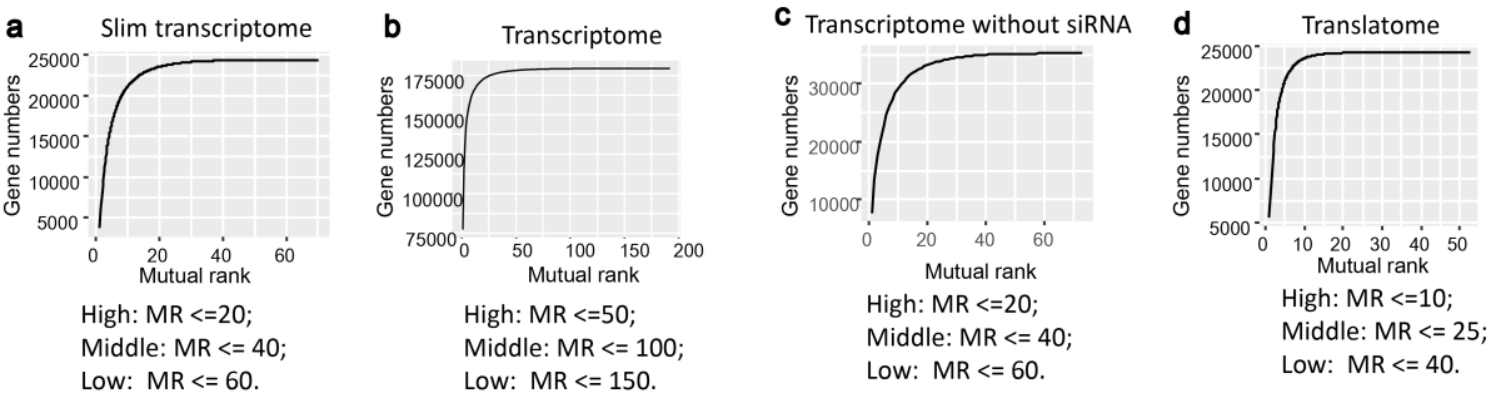

Supplementary Fig. translatomic interactions. a-d. Saturation-levels across different mutual ranks of coexpression relationships and cutoffs for the identification of high-confidence, middleconfidence, and low-confidence co-expression networks. 

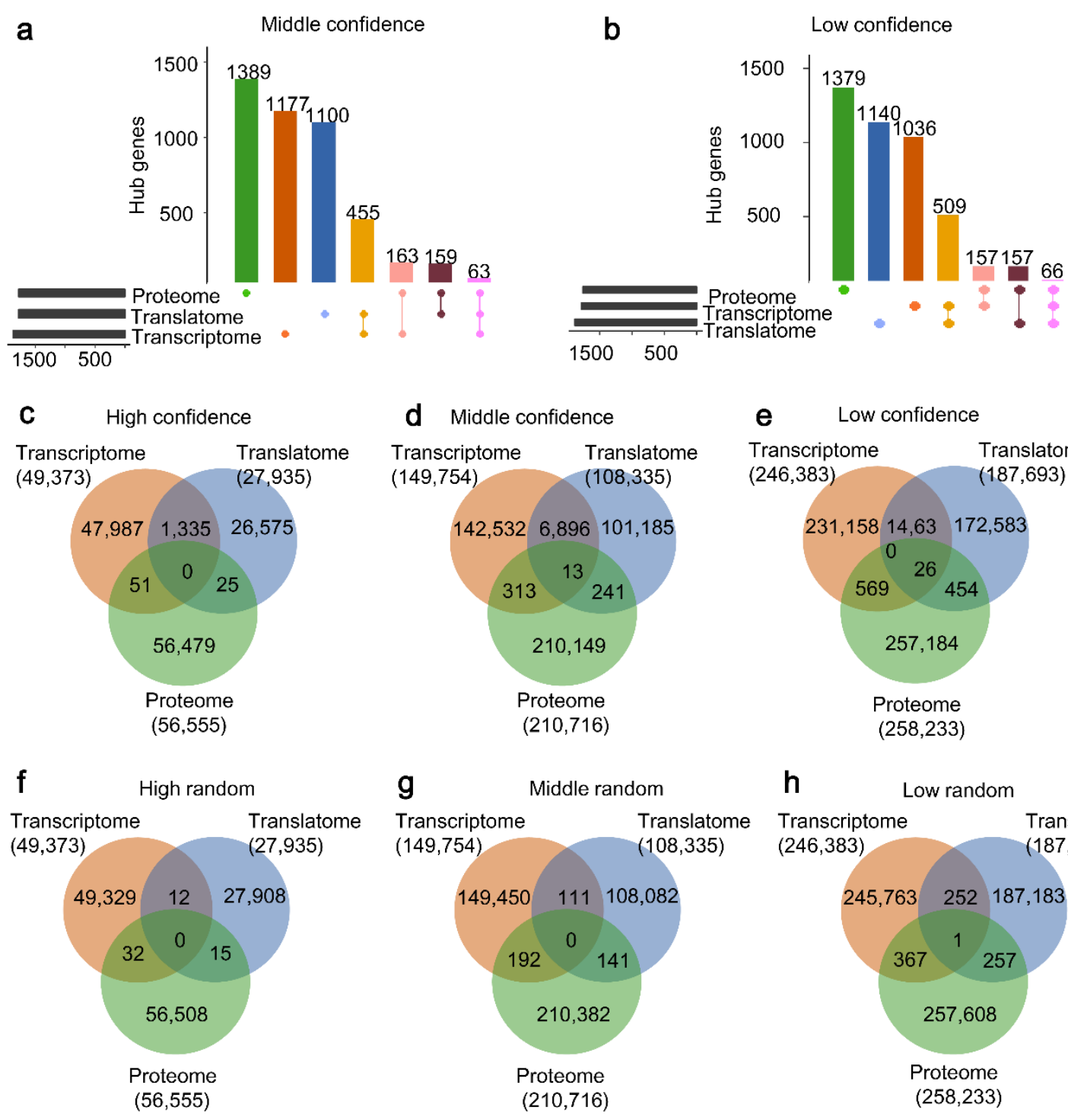

d Middle confidence

Transcriptome Translatome $(149,754)$

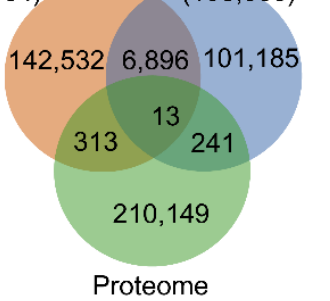

$(210,716)$

g

Transcriptome $(149,754)$

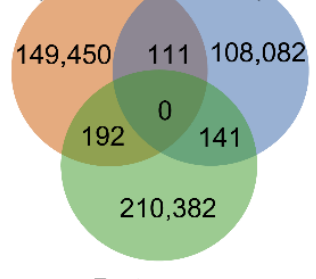

Proteome $(210,716)$

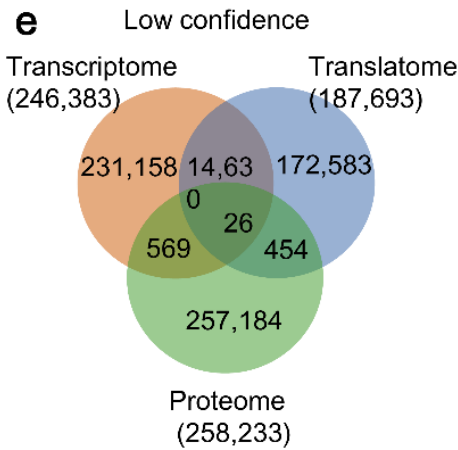

h Low random $\begin{array}{ll}\text { Transcriptome } & \text { Translatome } \\ (246,383) & (187,693)\end{array}$

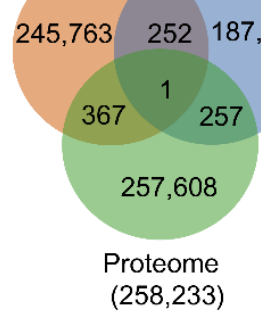

Supplementary Fig. 4. Overlapping of hub genes and edges between different omic transcriptome, translatome, and proteome at middle- and low-confidence levels. c-h. Shared edges between transcriptome, translatome, and proteome in our study and the randomly generated interaction datasets at all different confidence levels. The sharing rates of edges in our study (c, d, e) is significantly higher than that in the randomly 

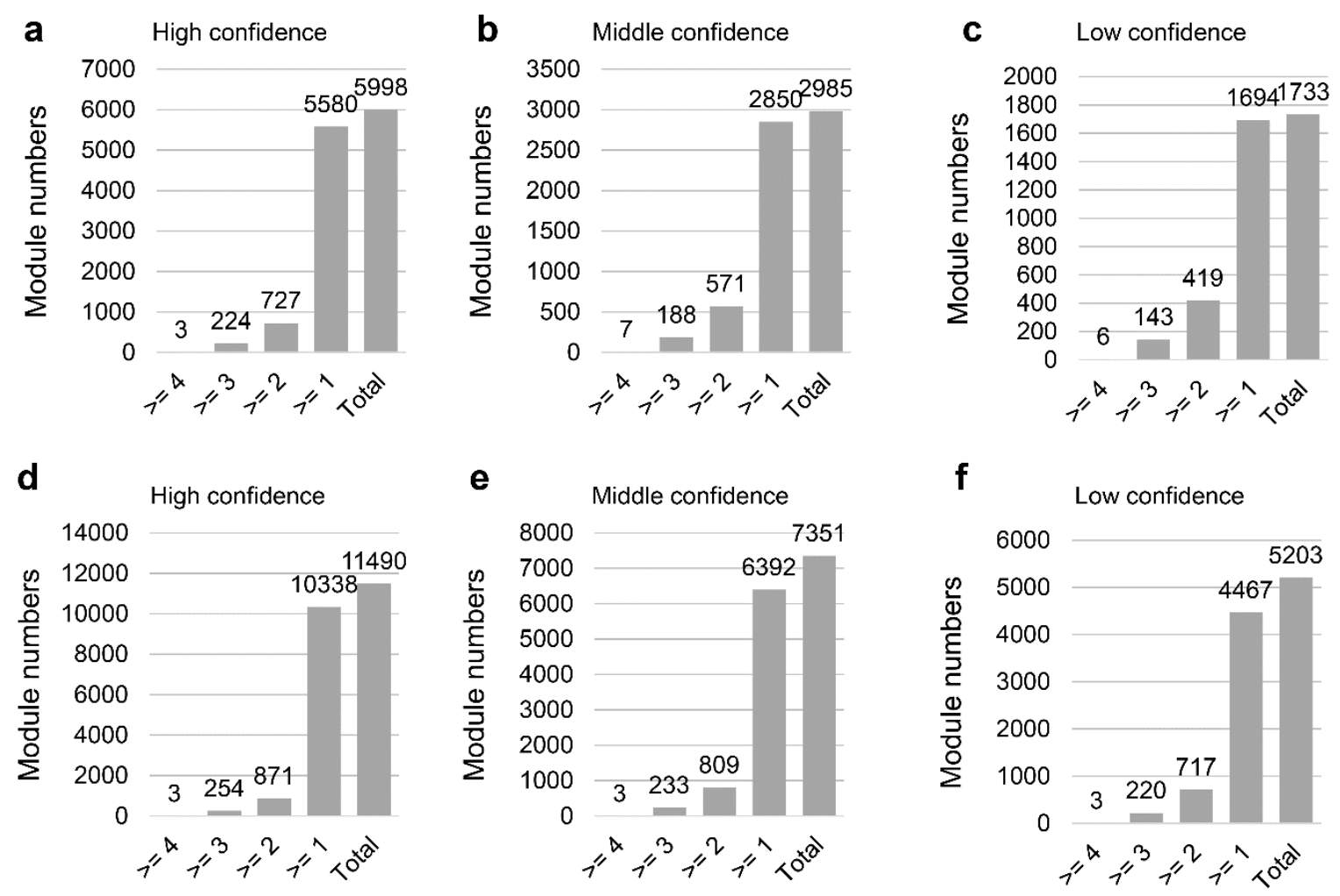

918 Supplementary Fig. 5. The numbers of modules which contain types of functional elements in transcriptome and integrative networks. The $\mathrm{x}$-axis represents how many different types of functional elements are contained in the module and the total number 


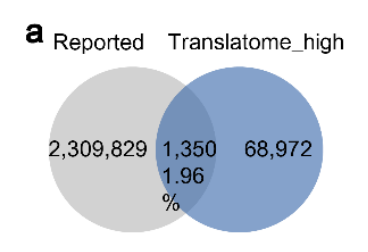

d Reported Proteome_high

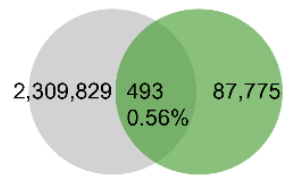

g Reported Slim-

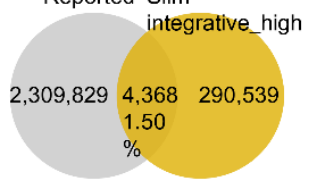

$\mathbf{k}$
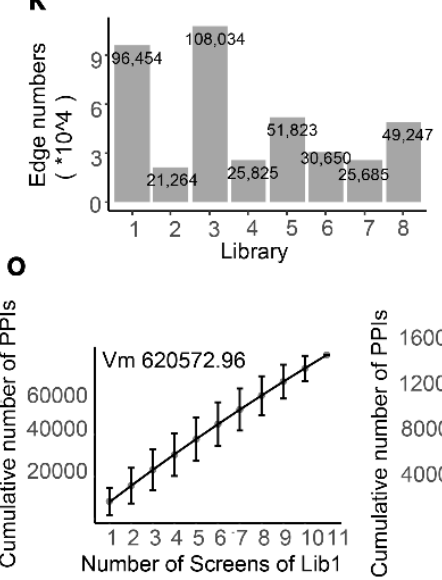

焉 b Reported Translatome_middle

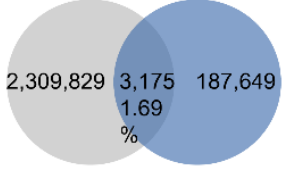

e Reported Proteome_middle

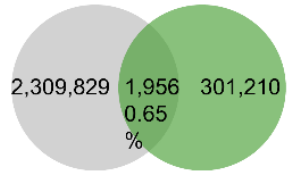

h Reported Slim-

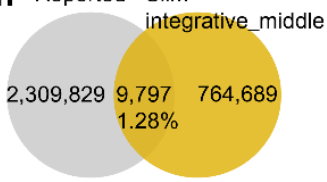

\section{I}

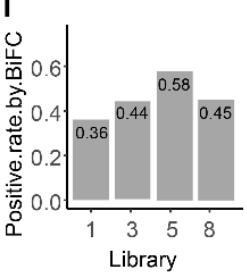

C Reported Translatome_low

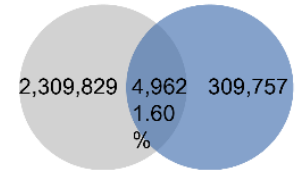

Reported Proteome_low
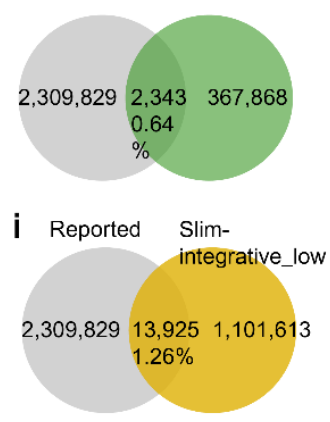

j Reported Random

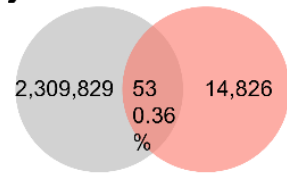

m
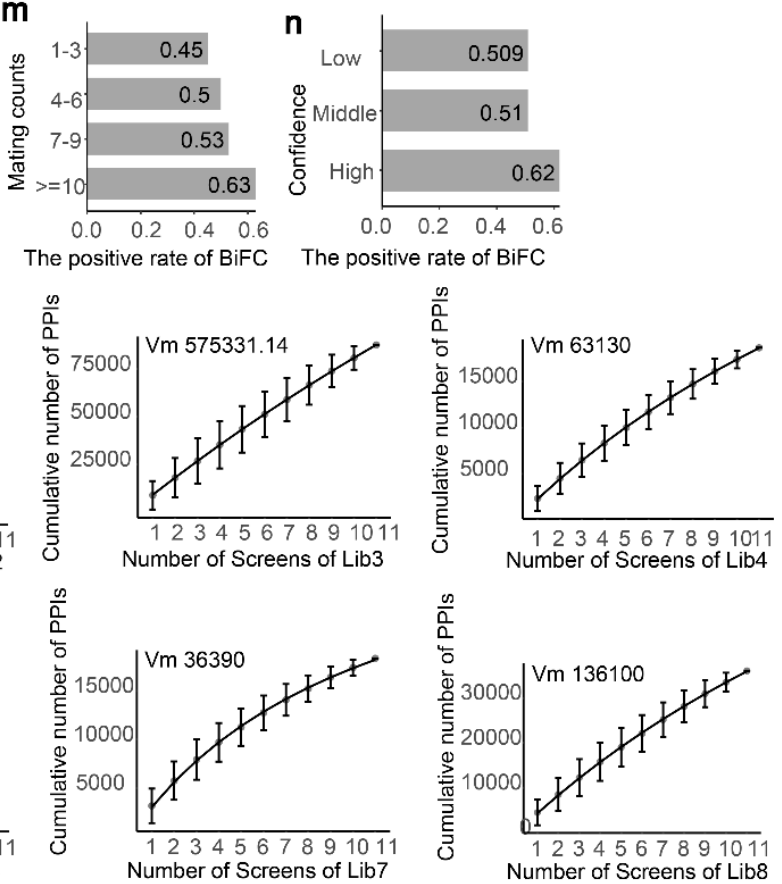

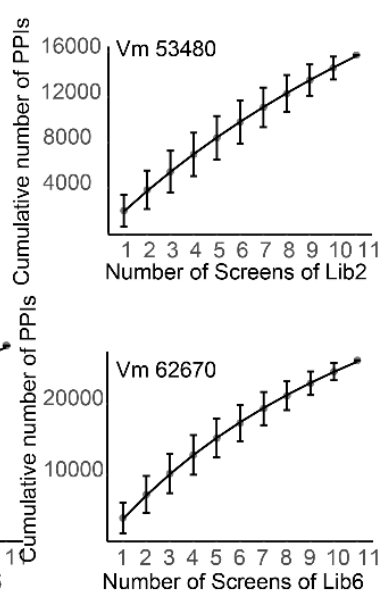

Supplementary Fig. 6. Overlapping between reported interactions and our current saturation curve for each library across different numbers of mating. 
927 Supplementary Fig. 7. Validation of randomly selected PPIs by BiFC.

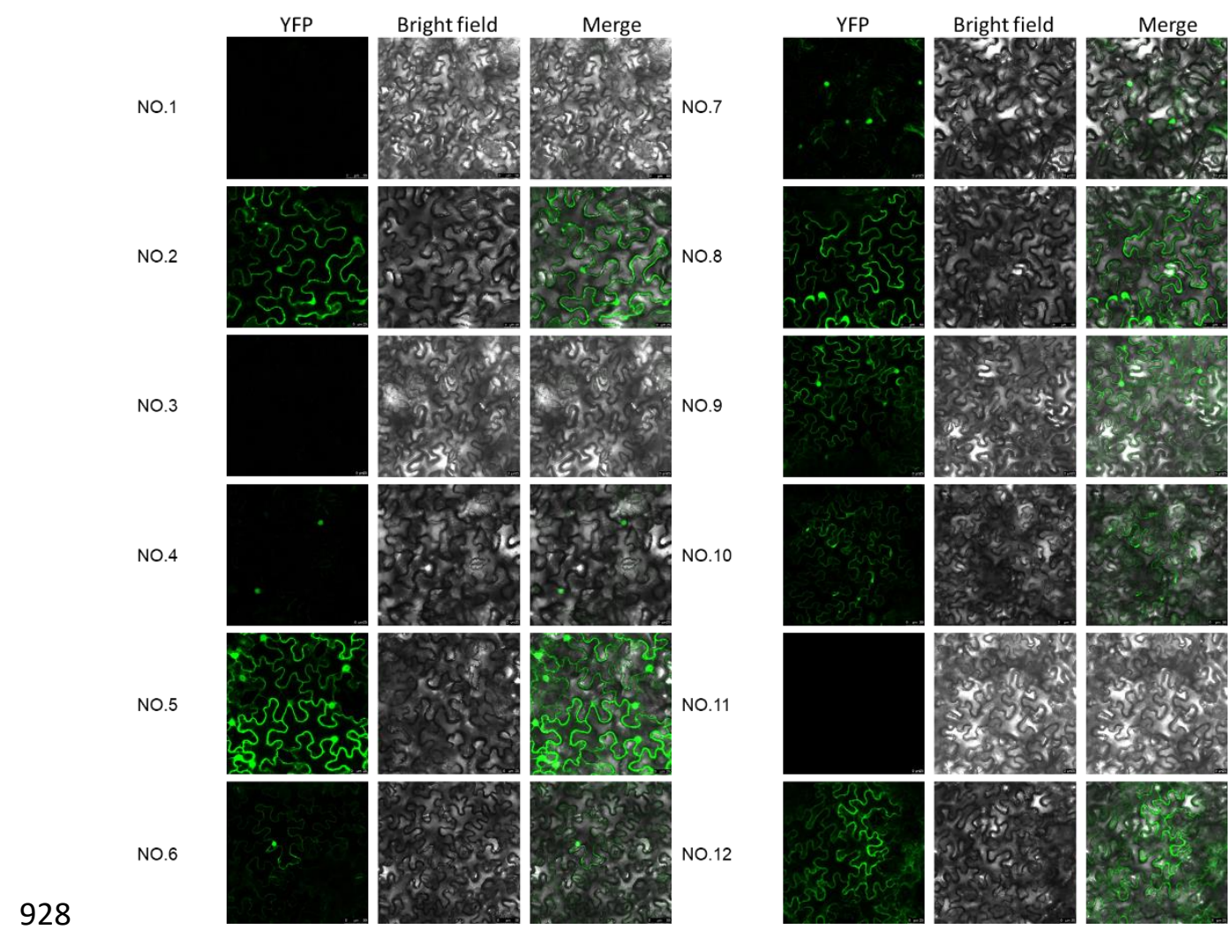




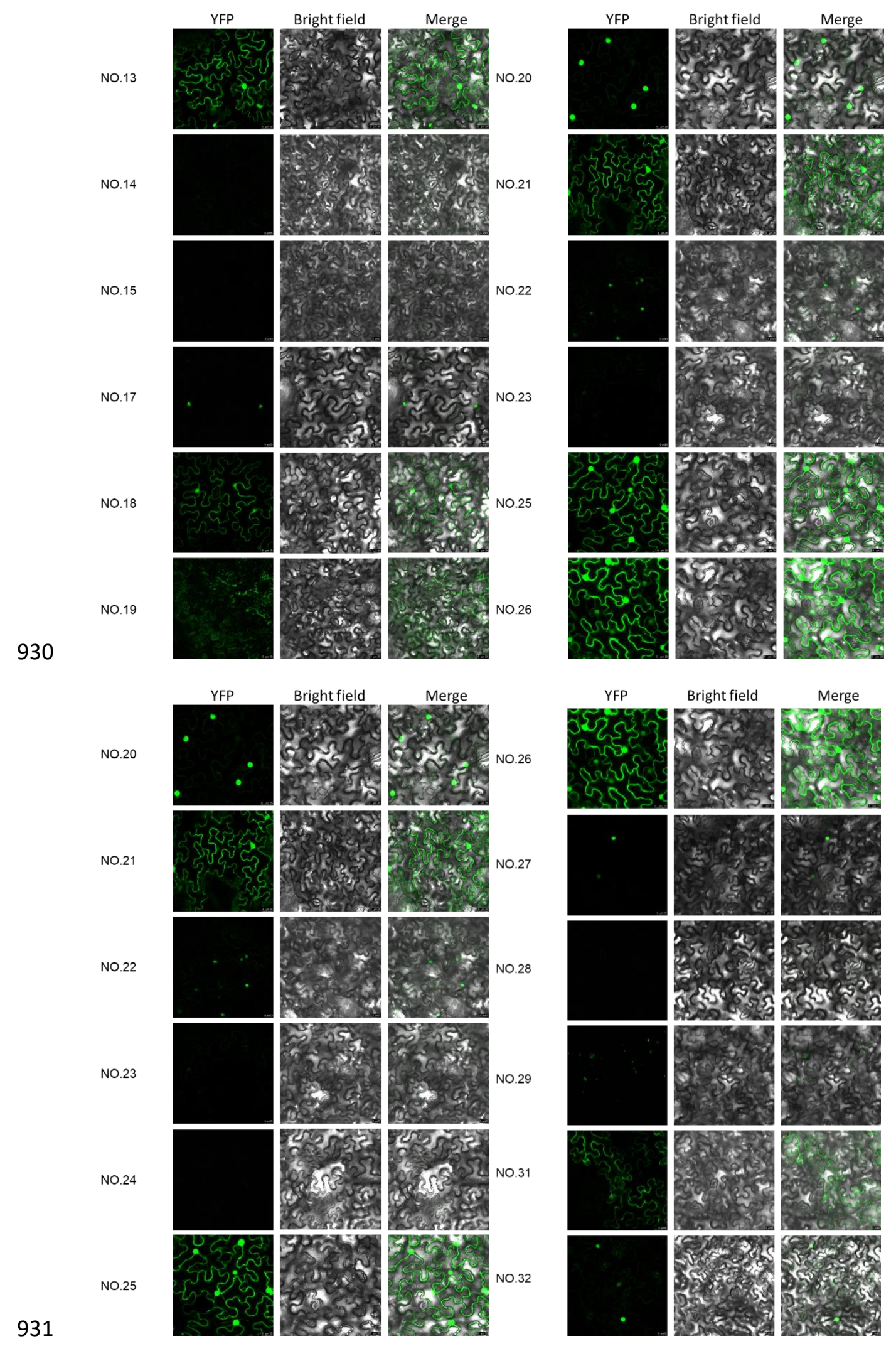




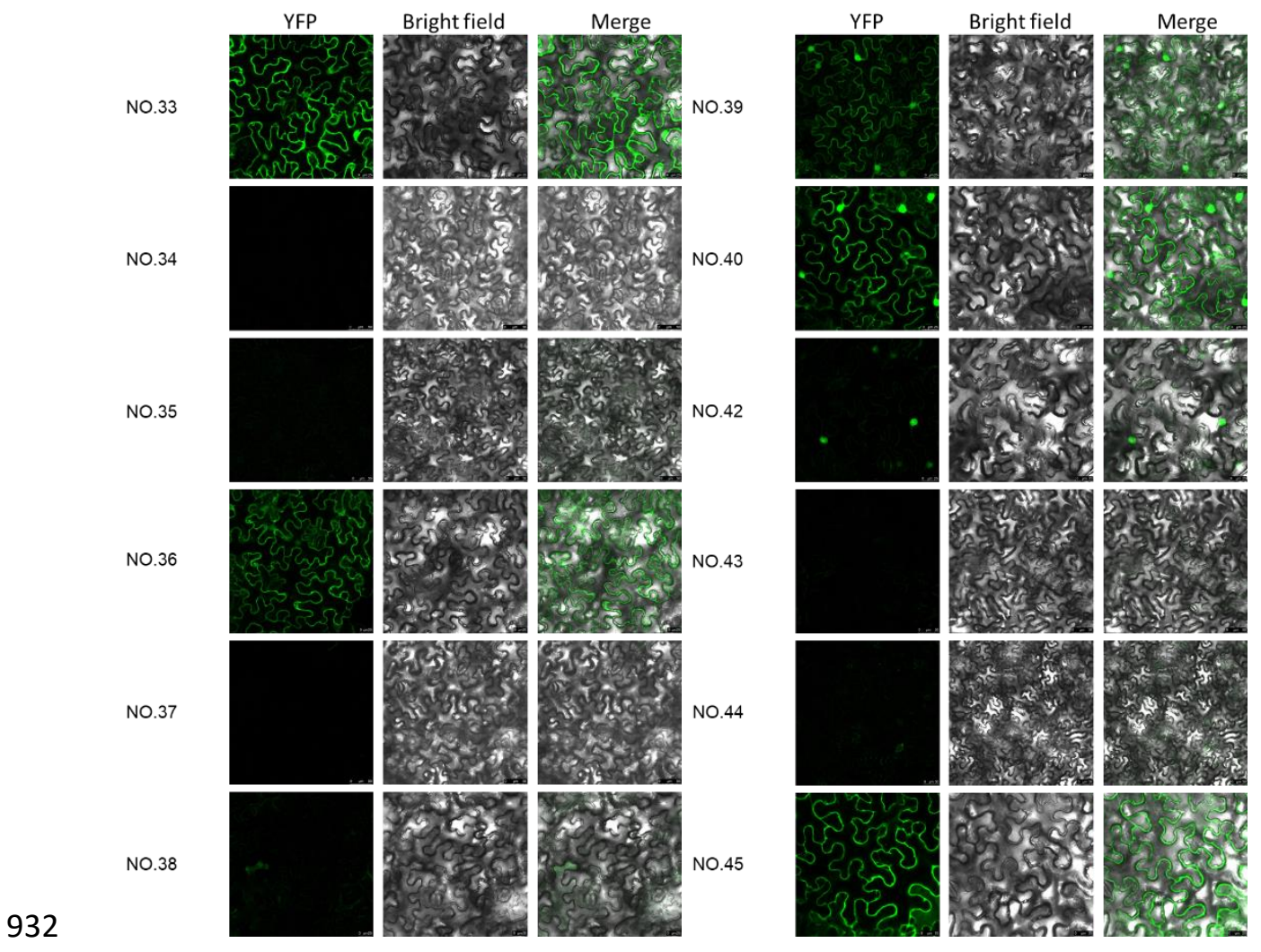

933

934 


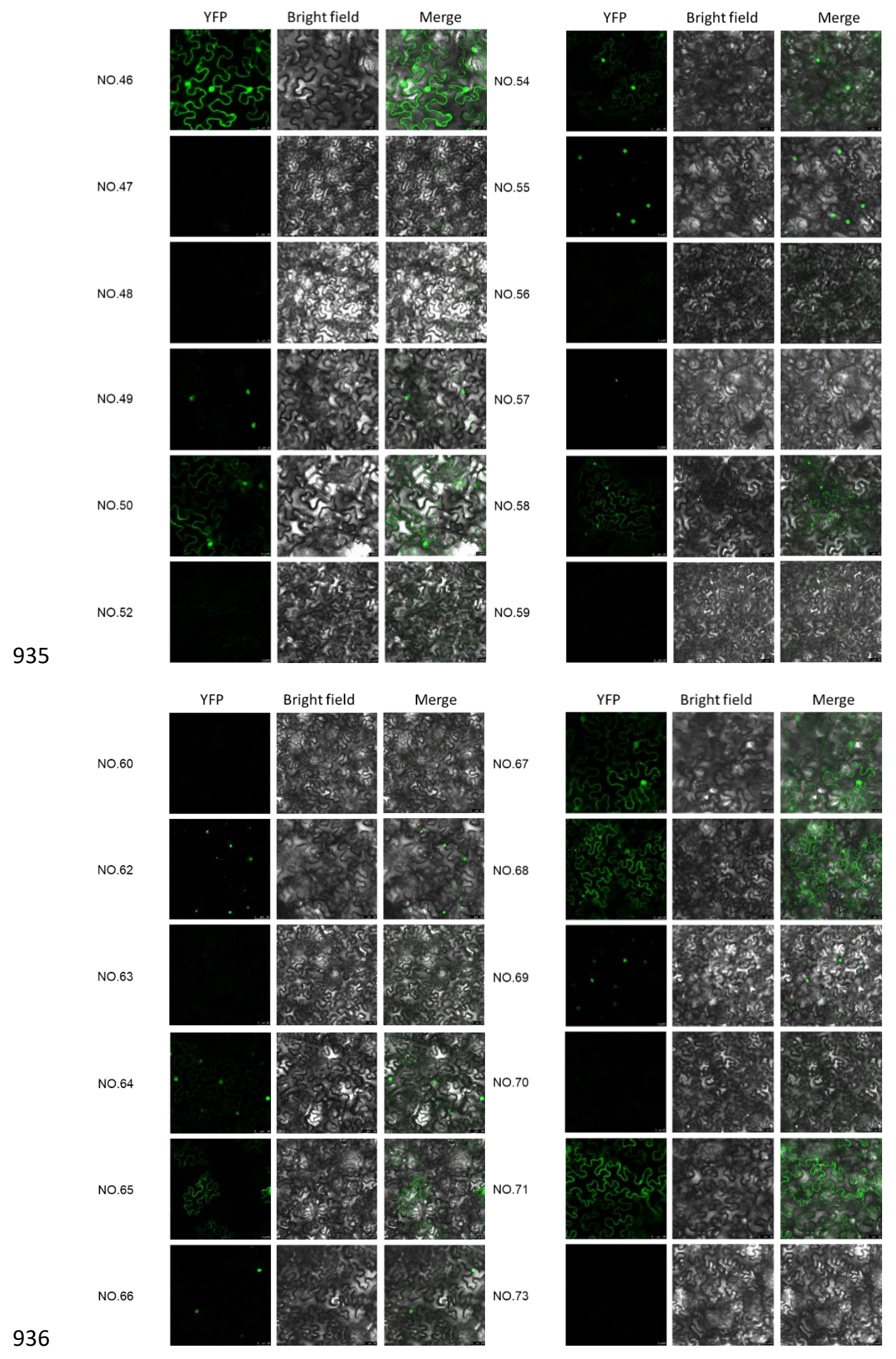




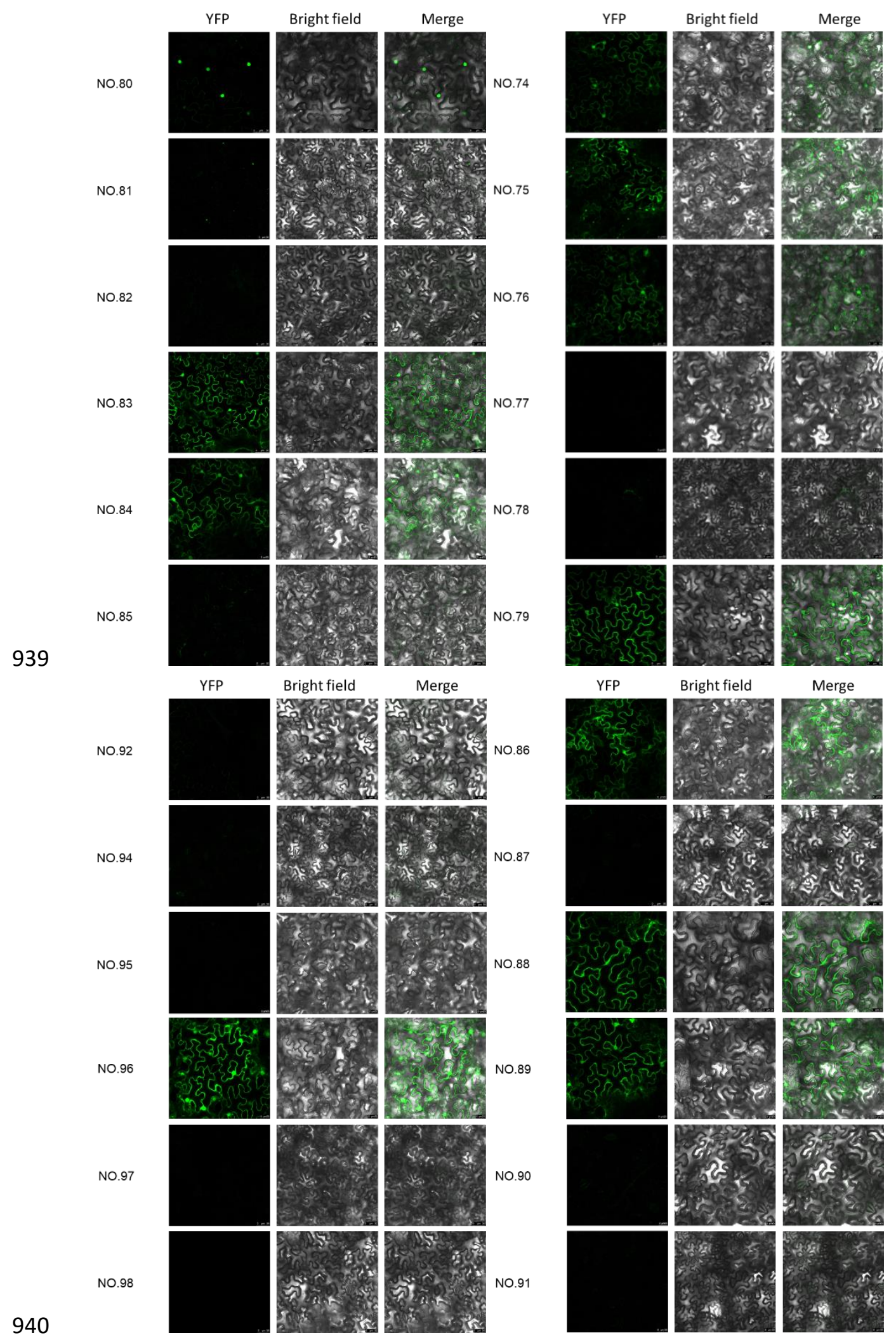




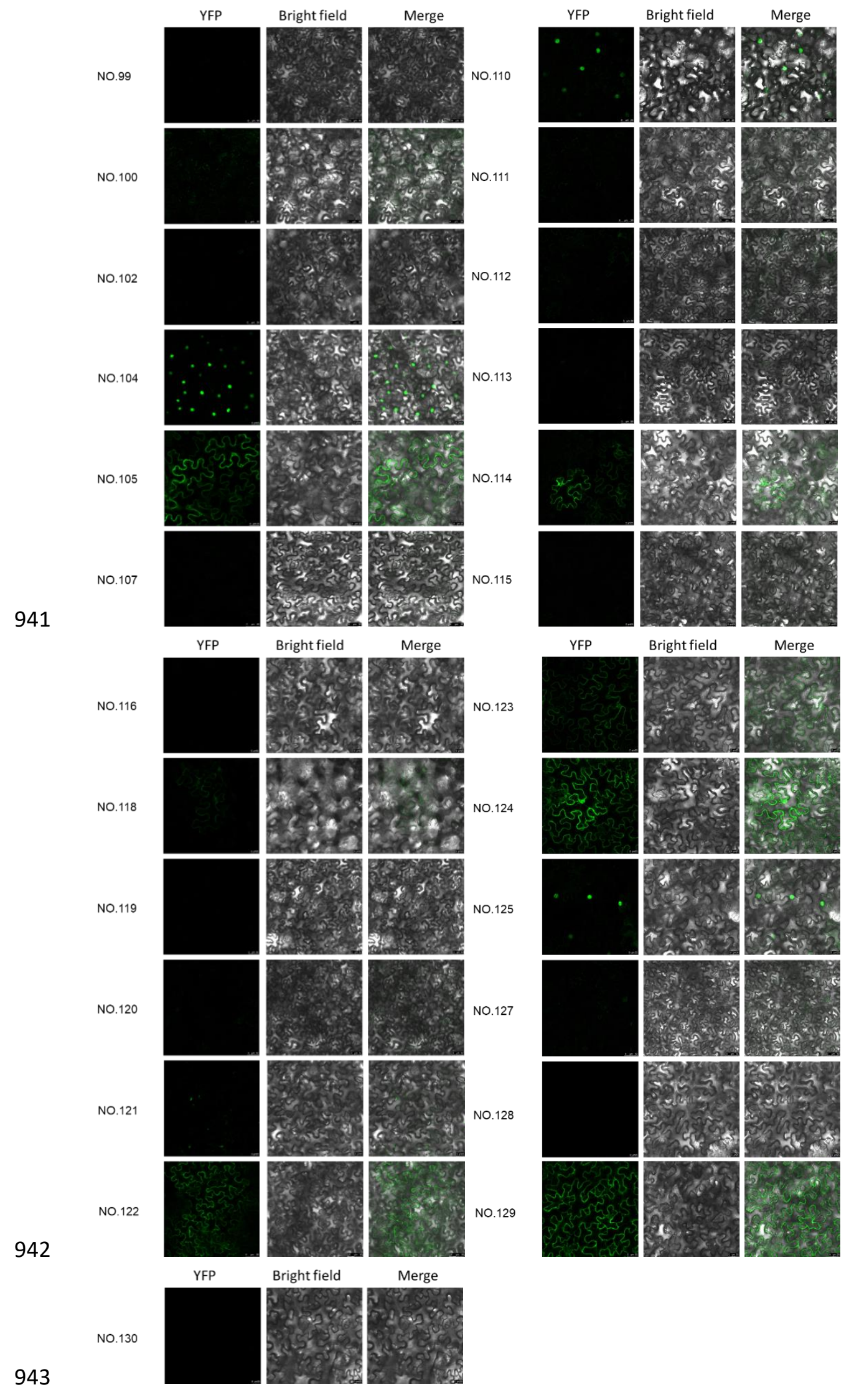


a

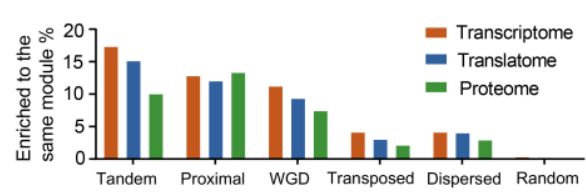

b

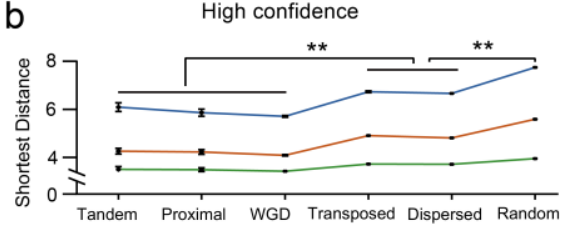

C

$\circ^{20} 7 \quad$ Middle confidence $\square$ Transcriptome

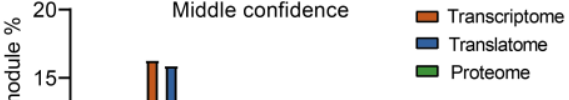

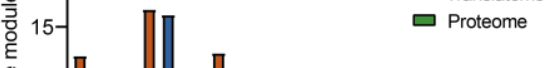
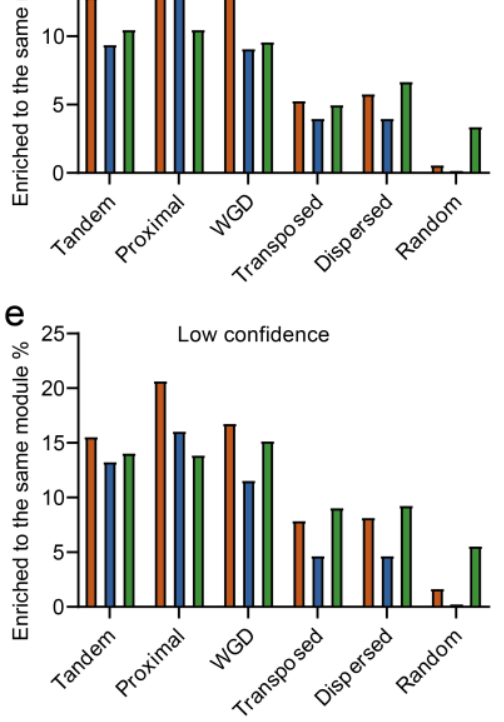
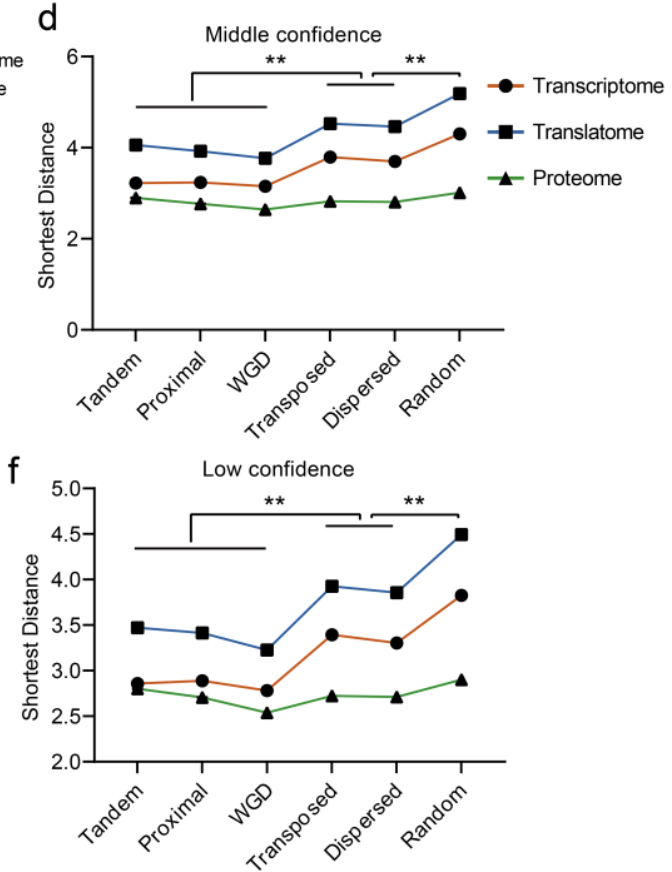

g
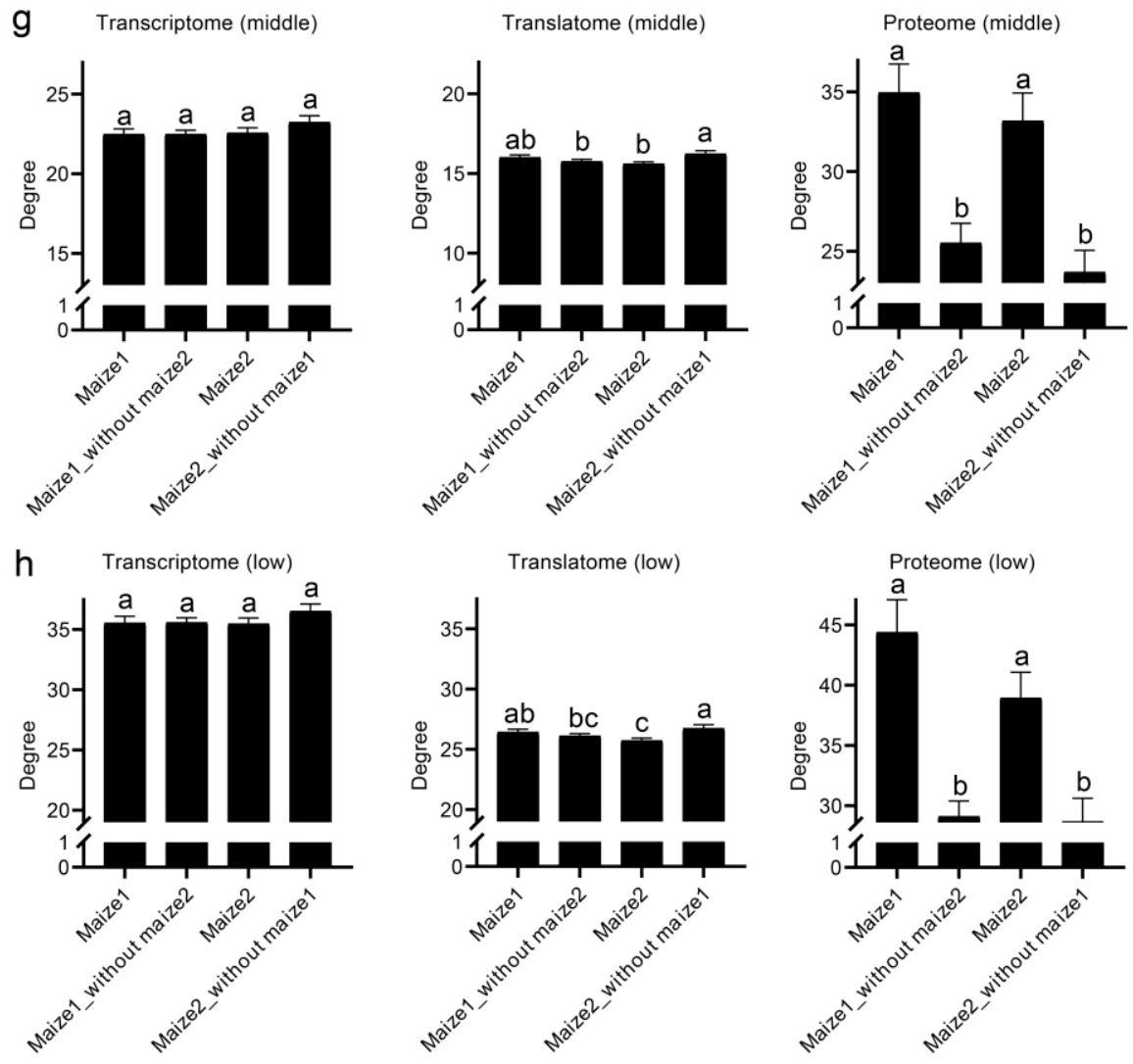
945 Supplementary Fig. 8. Functional divergence between duplicate genes of different 946 origination types and progressive differentiation between two maize subgenomes

947 at middle- and low- confidence levels. a-f, The proportion of different types of 948 duplicate genes that are functionally clustered (left) in the same module across different 949 regulatory levels of high- $(a, b)$, middle- $(c, d)$ and low-confidences $(e, f)$ and distribution 950 of shortest distance (right) in different regulatory networks for different types of 951 duplicate genes of high-, middle- and low-confidences. g-h. Degree variation of maize subgenome genes across different regulatory levels of middle- and low-confidences. Maize1 and Maize2 represent homologous genes from subgenome maize1 and maize2, respectively. Maize1 without maize2 indicates subgenome maize1 genes, of which the corresponding maize2 genes lost. Maize2 without maize1 indicates subgenome maize2 

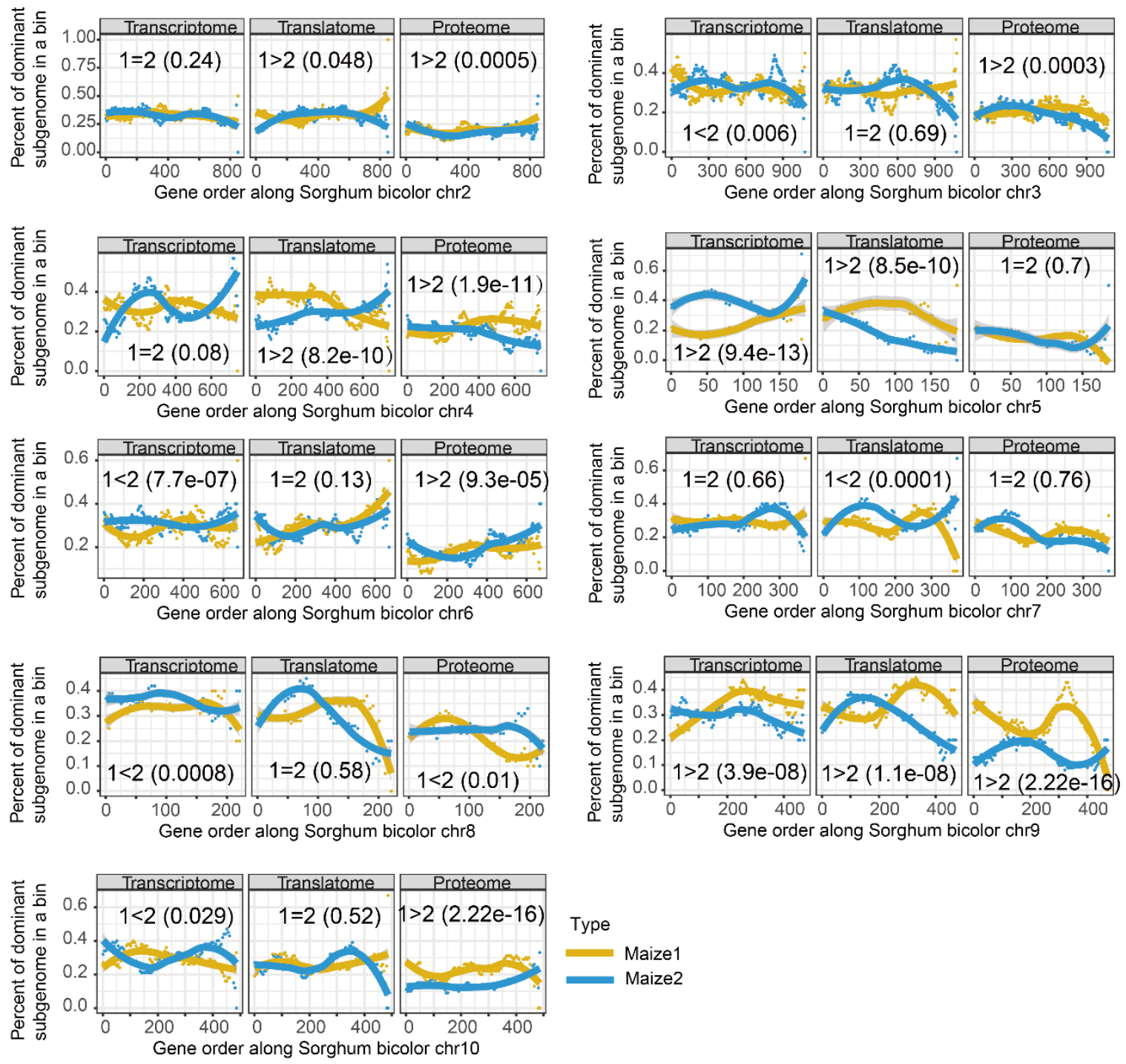

Type
Maize1
Maize2

Supplementary Fig. 9. Biased regulatory fractionation at transcriptomic, 
a

963

964

965

966

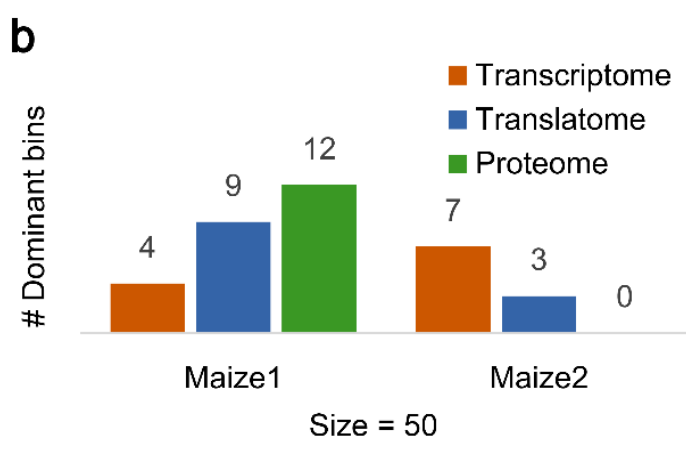

Supplementary Fig. 10. Number of sliding window bins (at different step sizes of 10 and 50) showing significant functional divergence between two subgenomes. 

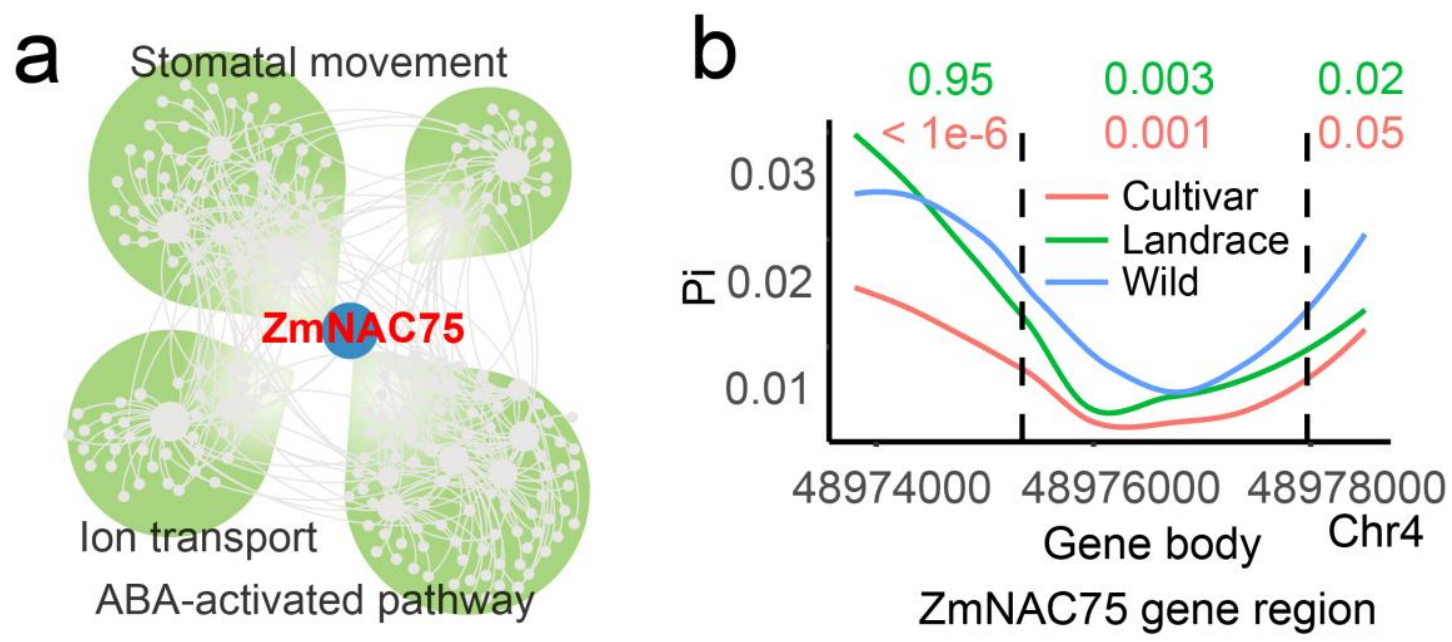

Supplementary Fig. 10. Maize2 hub gene $Z m N A C 75$ was subject to selection during maize improvement. a, A regulatory network of maize 2 hub gene $\mathrm{ZmNAC75}$ at the transcriptome level. ZmNAC75 cross-talks to four regulatory modules, of which three have significant gene ontology enrichment for ABA activated pathway, stomatal movement, and ion transport, respectively. b, Nucleotide diversity across ZmNAC75

976 
a

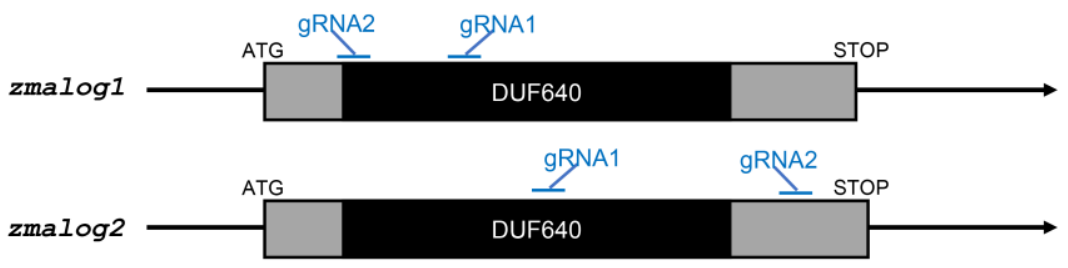

WT: CGCCGTCGGTTGGTTCCATGACGCCGCA (N78) GCTGAGCCTGGCGCAGTGCAGCGGGGCT

zmalog1-1: CGCCGTCGGTTGGTTCCA---124bp del-------

WT: TGCCCTTTCTTCGGCCACCCGGCGCCGC (N233) GCCGTCATCTCCTCCTCCCACGACGGCA

zmalog2-1: TECCTTTCTTC----ACCCGGCGCCGC (N233) GCCGTCATCTCCTCCTCCCACGACGGCA

zmalog2-2: TGCССTTT----GGCCACCCGGCGCCGC (N233) GCCGTCATCTCCTCCTCCCACGACGGCA

Red: gRNAs, green: Pam site, blue: insertion

b
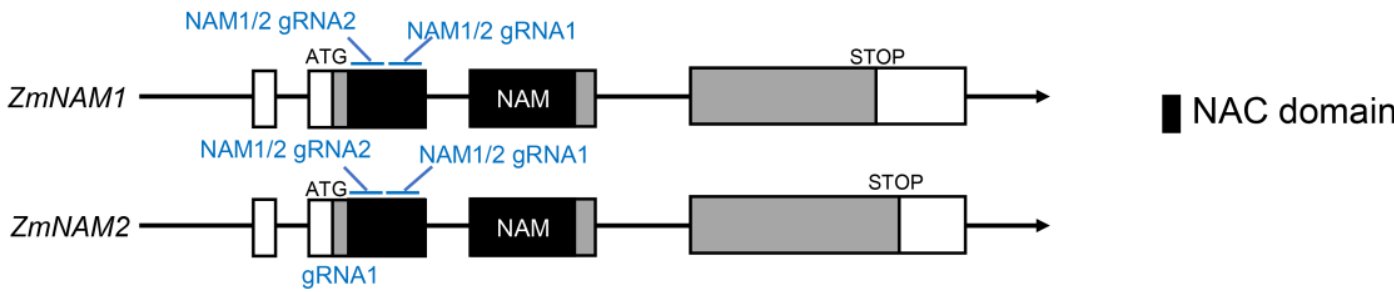

NAM1/2-gRNA2 $40 \mathrm{bp} \quad$ NAM1/2-gRNA1

WT: TCCACCCGACGGACGAGGAGCTCATCACCTA//CGCGCCATCGCGGAGATCGACCTGAACAAGTGCG

Zmnam1-1: TCCACCCGACGGACGAGGAGCTCATCACCTA//CGCGCCATCG-GGAGATCGACCTGAACAAGTGCG

Zmnam1-2: TCCACCCGACG------------------------CGGAGATCGACCTGAACAAGTGCG 70bp del

Zmnam1-3: TCCACCCGACGGACGAGGAGCTCATCACCTA//CGCGCCATCGTCGGAGATCGACCTGAACAAGTGCG

Zmnam1-4: TCCACCCGACG-ACGAGGAGCTCATCACCTA//CGCGCCATCGCGGAGATCGACCTGAACAAGTGCG

Zmnam1-5: TCCACCCGACGGACGAGGAGCTCATCACCTA//CGCGCCATCG--------ACCTGAACAAGTGCG 9bp del gRNA1

WT: CGGCCGCGCCATCGCGGAGATCGACCTGAACAAGTGCGAG

Zmnam2-1: CGGCCGCGCCATC--GGAGATCGACCTGAACAAGTGCGAG (deletion)

Zmnam2-2: CGGCCGCGCCATCGGCGGAGATCGACCTGAACAAGTGCGAG (insert)

Red: gRNAs, green: Pam site, blue: insertion

990

991 Supplementary Fig. 12. Mutagenesis in zmalog1, zmalog2, nam1 and nam2. 
a

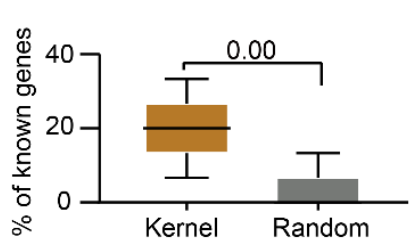

b

Zm00001d003047

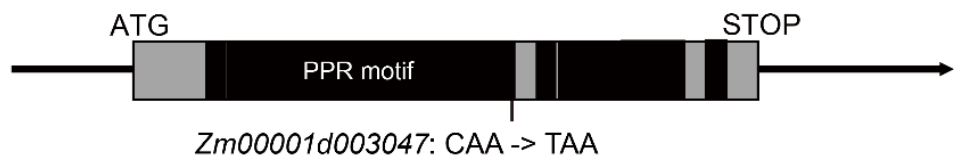

994

995

996

997

998

999

1000

1001
Supplementary Fig. 13. The prediction rate of regulatory network extracted from the draft interactome by a randomly selected subset of well-known kernel functional genes (Kernel) and by a randomly selected genome-wide genes (Random) as queries. a, The constructed subnetworks of 40 randomly selected kernel genes (1000 time simulations) could predict up to $40 \%$ of the validation kernel genes, which is significantly higher $(\mathrm{P}=0.00)$ that of randomly selected function-unrelated genes. $b$, Mutagenesis in Zm00001d003047. 
a

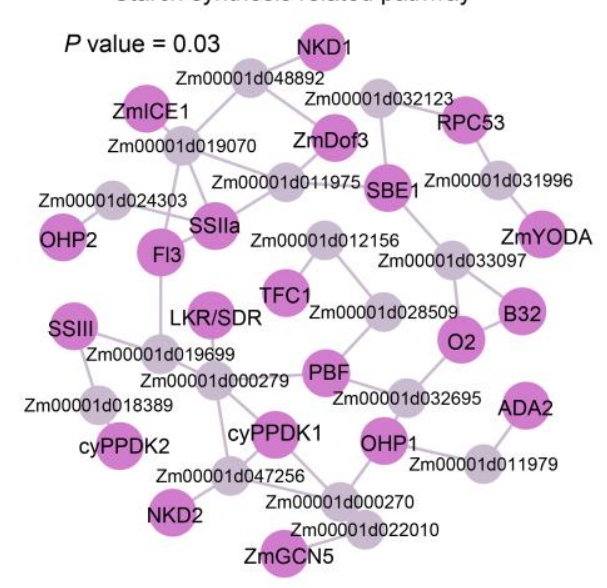

C

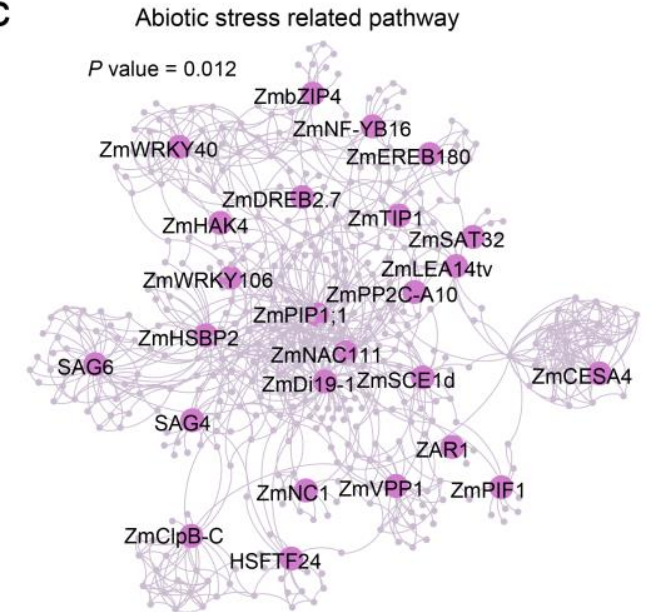

b Meristem developmental related pathway

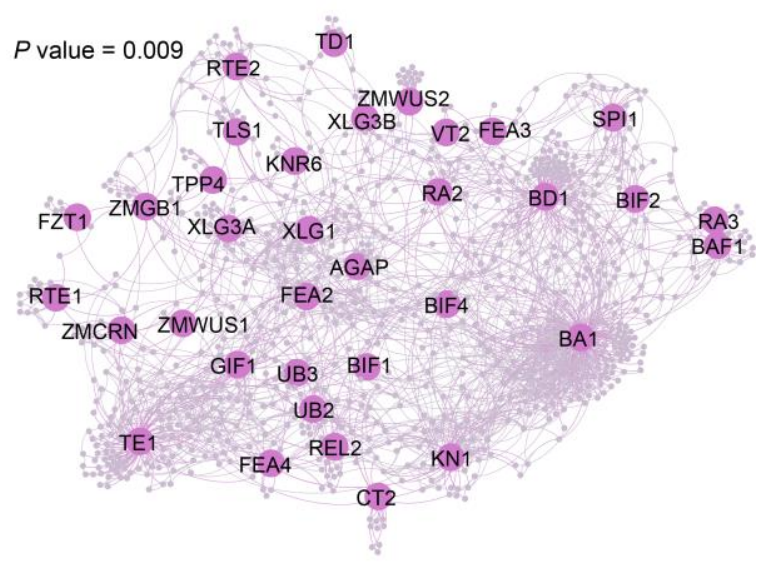
pathways in maize. a, Reconstruction of the regulatory network for starch synthesis pathway in maize. b, Reconstruction of the regulatory networks for meristem related genes. 


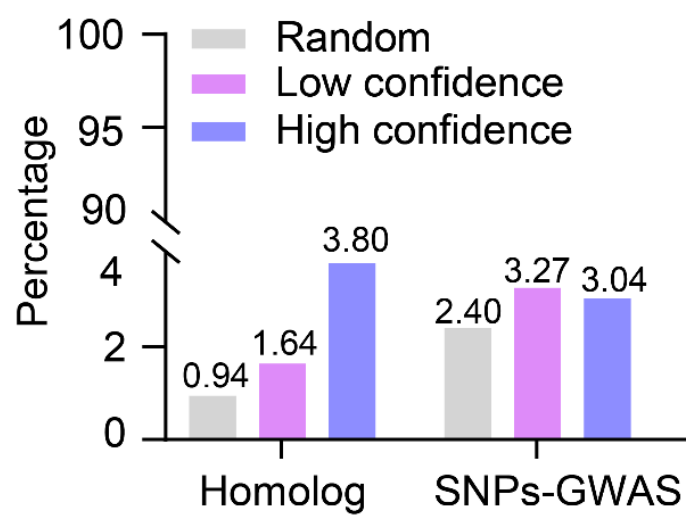

1010 Supplementary Fig. 15. Percentage of predicted flowering genes supported by other related flowering time evidence. Homolog means genes that are homologs to Arabidopsis FT genes identified in flowering time database (http://www.phytosystems.ulg.ac.be/florid/). SNPs-GWAS means genes with FT association signals identified in maize by Liu et al., $2020^{34}$. 


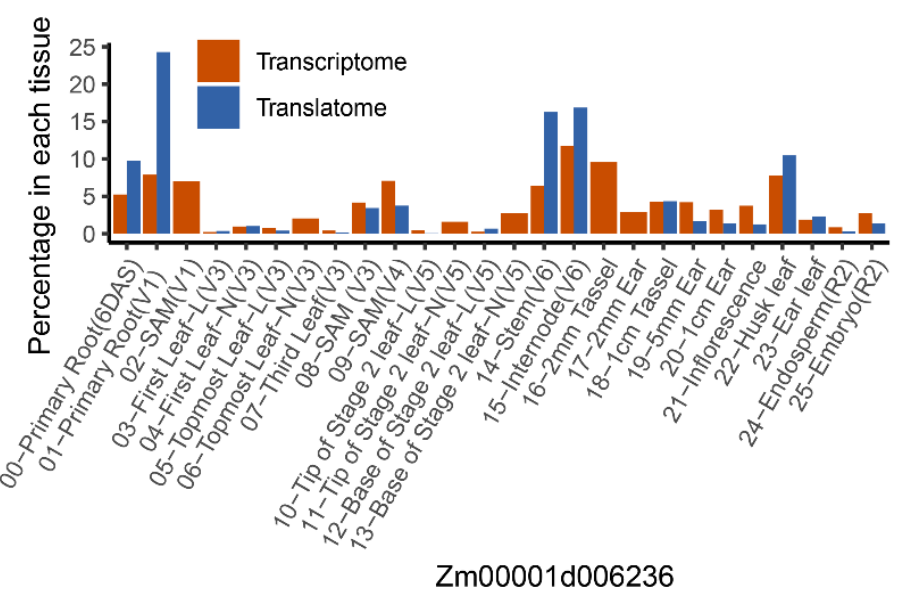

1018 Supplementary Fig. 16. Expression of predicted FT gene Zm00001d006236.

1019

1020 

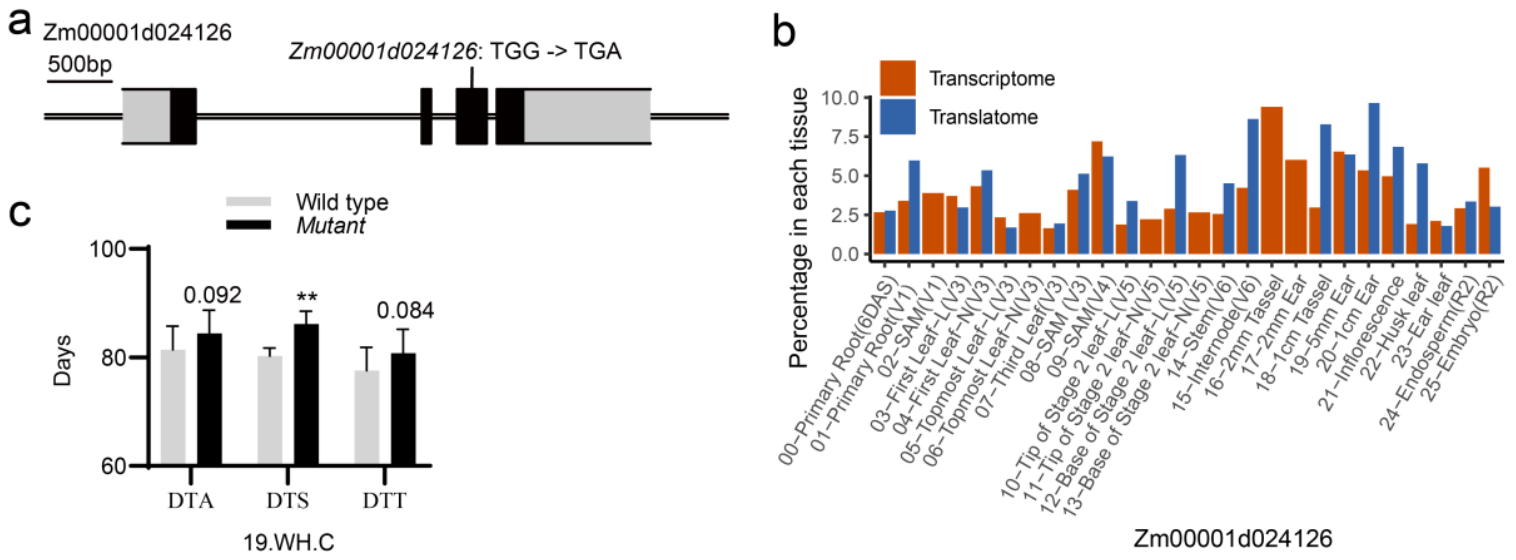

Supplementary Fig. 17. Functionally validation of predicted FT genes Zm00001d024126 by EMS mutant. a. An early stop codon was introduced in the exon region of the gene Zm00001d024126. b. Expression-level variation of the FT gene at both transcriptome and translatome levels. c. Significant FT variation between wildtype and mutant at growing locations and years. The $\mathrm{x}$-axis is displayed by year.location.season. $\mathrm{C}$ means spring. \{masked because of the Double-blind peer review.\} DTT means days to tassel. DTA means days to anthesis. DTS means days to silking. At least five individual plants per genotype in the field have been used for the test of phenotypic variation. 


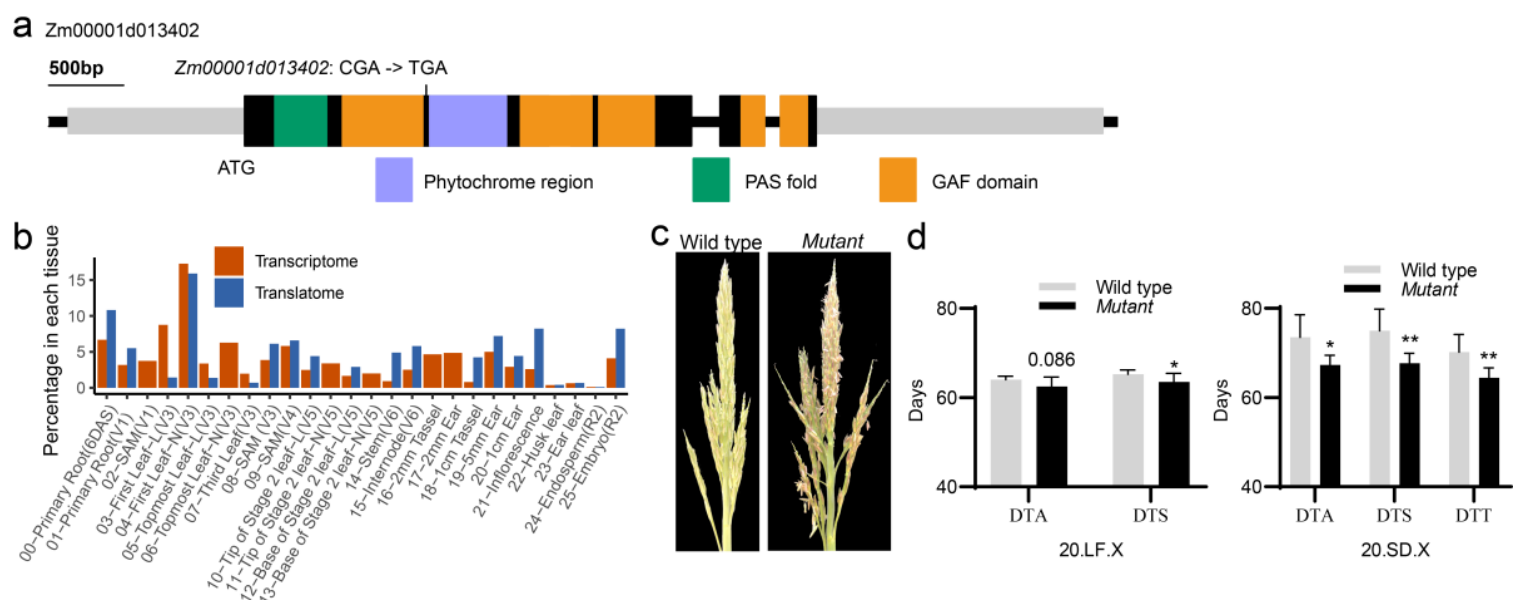

1033 Supplementary Fig. 18. Functionally validation of predicted FT genes 1034 Zm00001d013402 by EMS mutant. a. An early stop codon was introduced in the exon

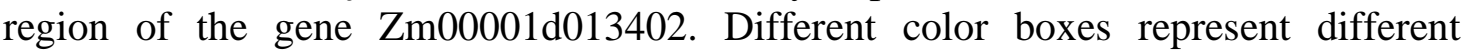
functional domain annotated by Interproscan. b. Expression-level variation of the FT gene at both transcriptome and translatome levels. c. Flower time differs between wildtype and mutant. d. Significant FT variation between wildtype and mutant at different growing locations and years. The $\mathrm{x}$-axis is displayed by year.location.season. $\mathrm{X}$ means summer. \{masked because of the Double-blind peer review. DTT means days to tassel. DTA means days to anthesis. DTS means days to silking. At least five individual plants per genotype in the field have been used for the test of phenotypic variation. 
a

PHYB1: Zm00001d028905

500bp phyb1: CAG -> TAG

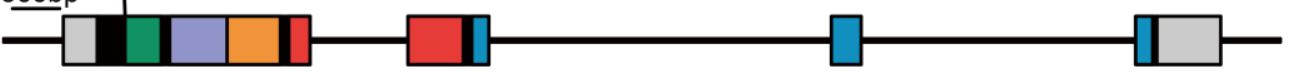

ПPAS_2 ПGAF ПPHY ПPAS ПHis_kinase_dom

b

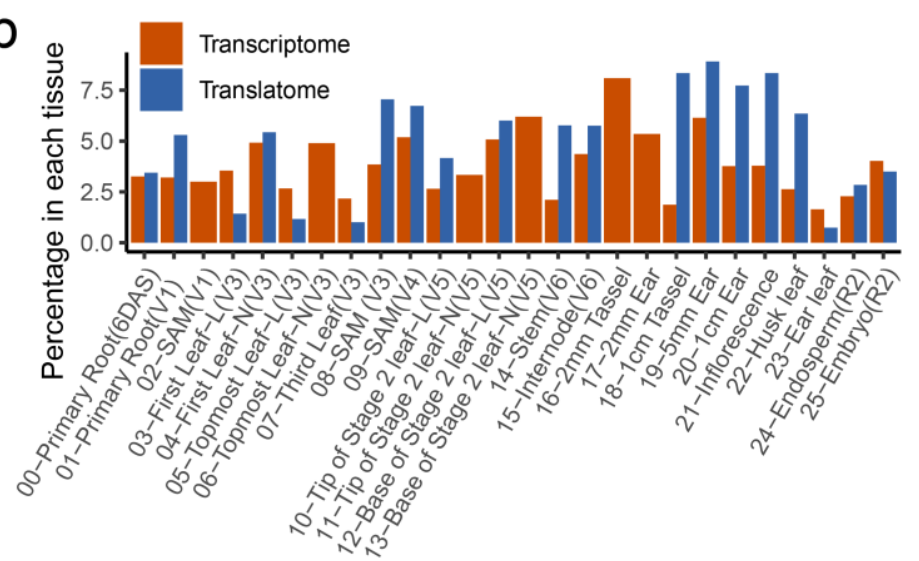

C Wild type Mutant
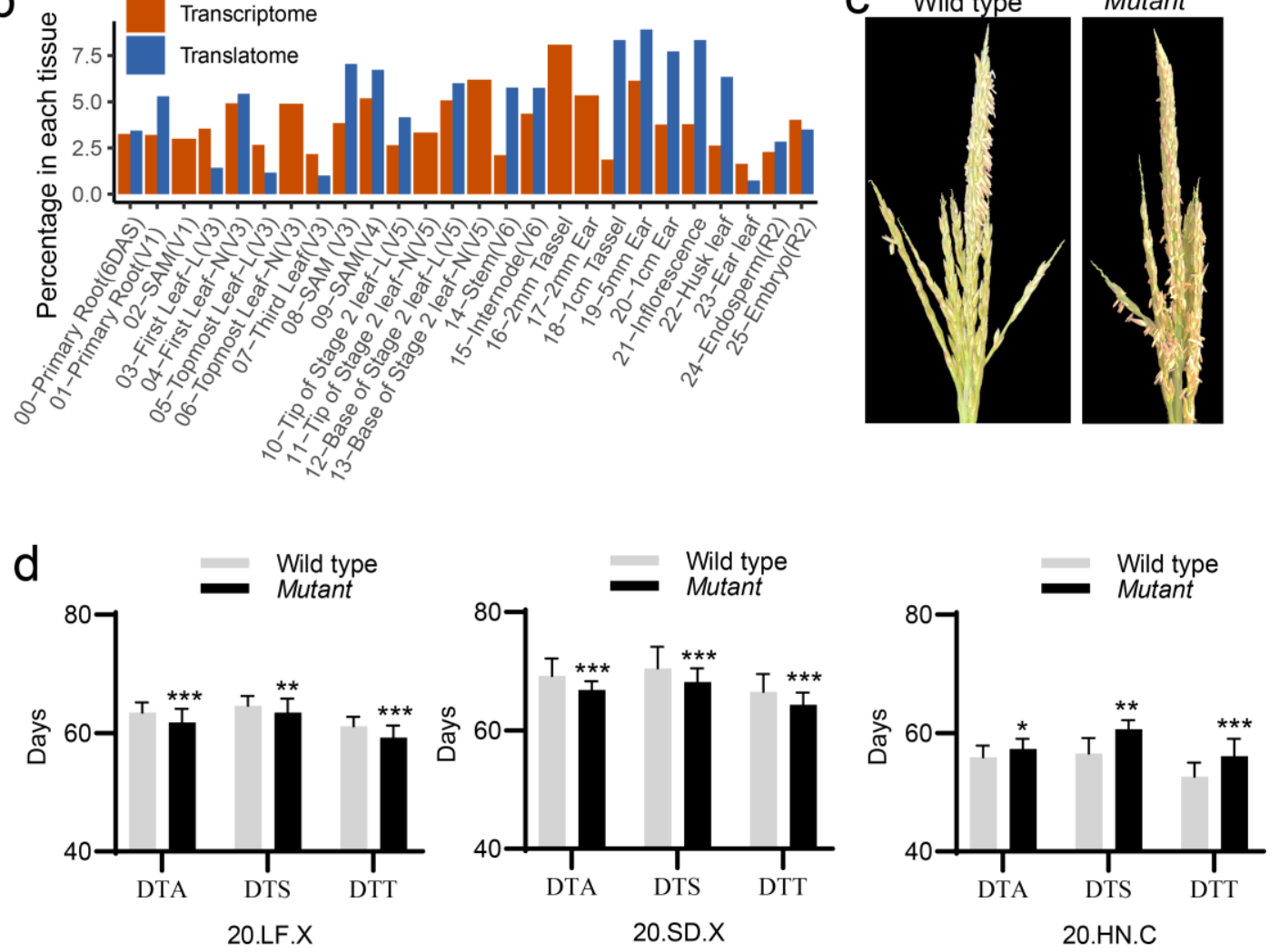

1046

Supplementary Fig. 19. Functionally validation of predicted FT genes Zmphyb1 by

EMS mutant. Legends are same to Supplementary Fig. 17. 
a $2 m 00001 d 033799$

$500 \mathrm{bp}$

ATG Zm00001d033799: CAG -> TAG

-

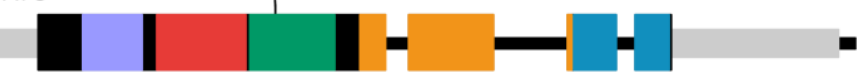

PAS_2 $\quad$ Phyto_chromo_attachment

b

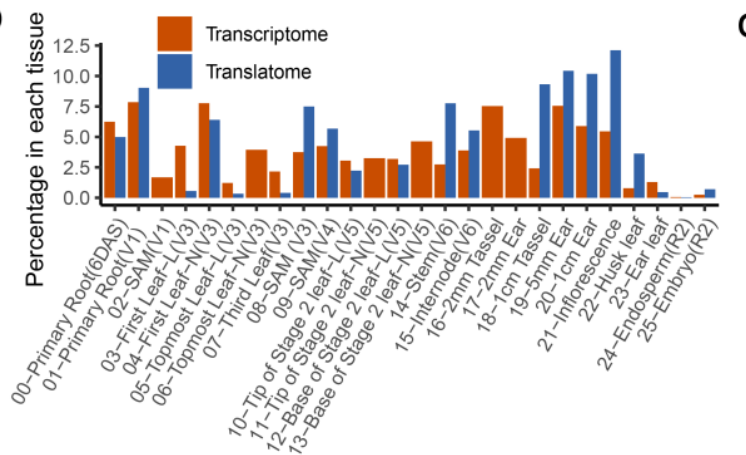

C PAS His_kinase_dom

Supplementary Fig. 20. Functionally validation of predicted FT genes 1051 Zm00001d033799 by EMS mutant. Legends are same to Supplementary Fig. 17. 
a ZmPHYC1: Zm00001d034038

$$
500 \mathrm{bp}
$$
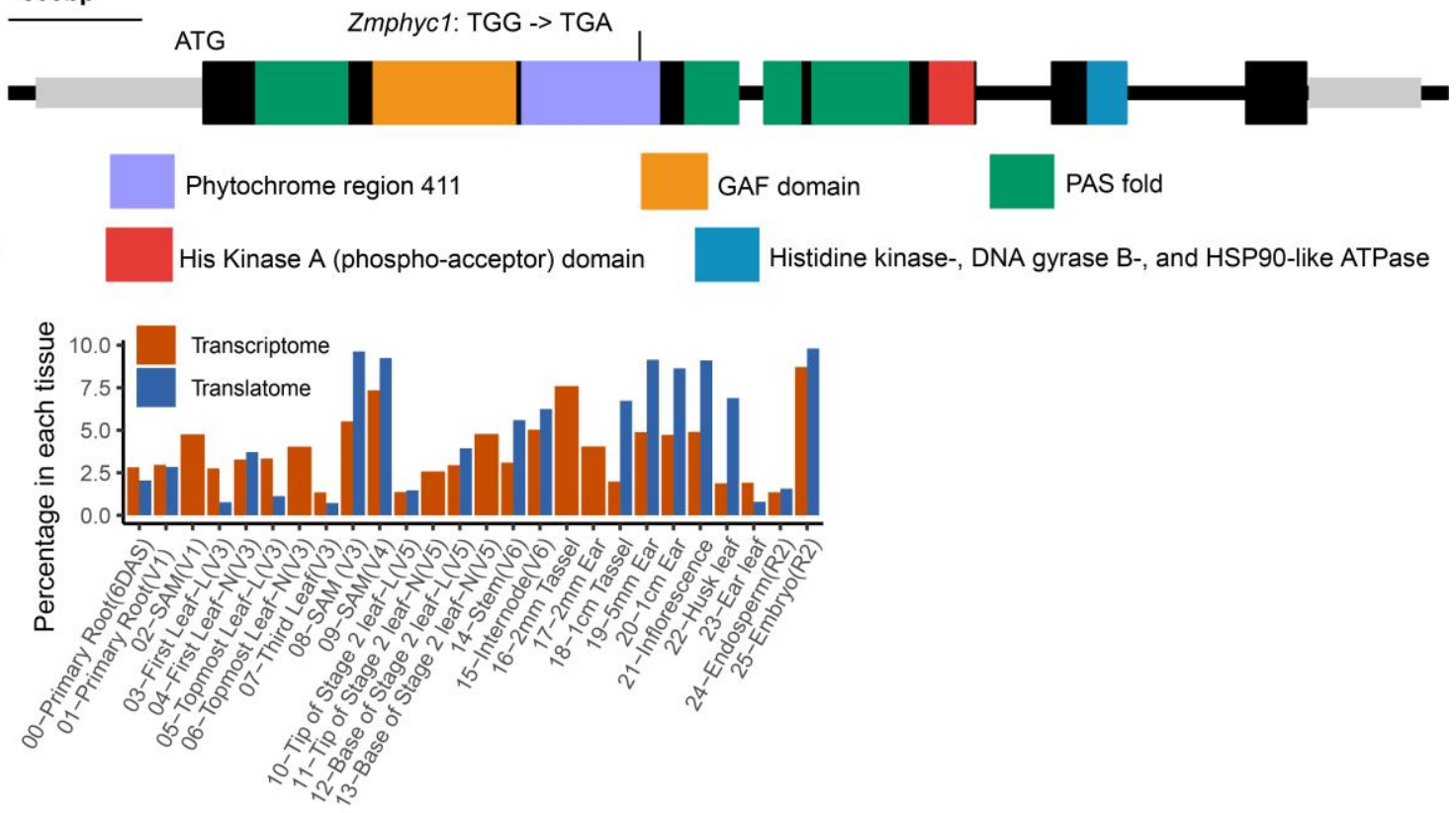

C
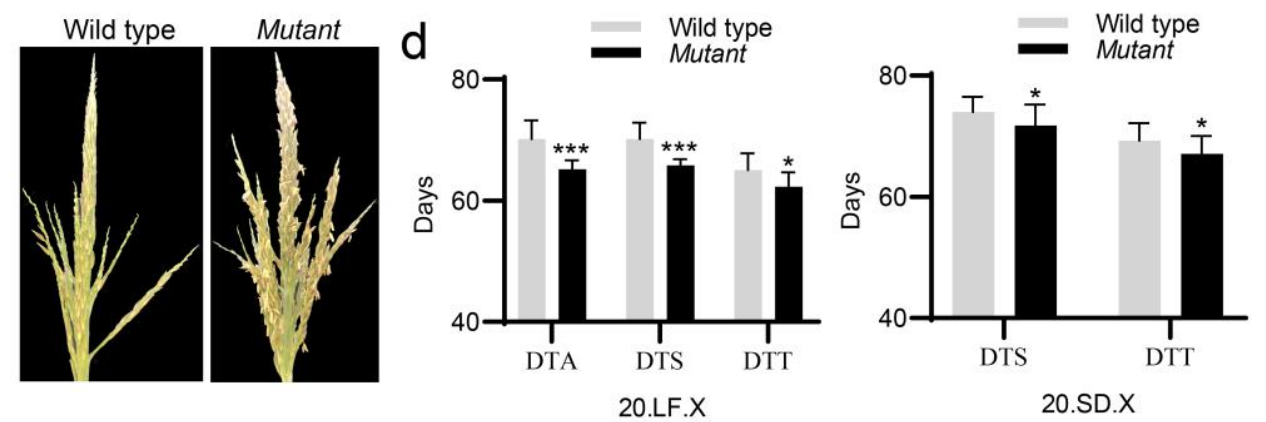
$a_{Z m 00001 d 045944}$

500bp Zm00001d045944: CGA -> TGA

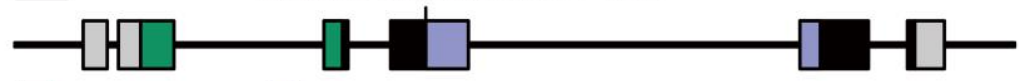

$\square$ DNA_photolyase $\square$ Cryptochr/Photolyase_FAD-bd

b
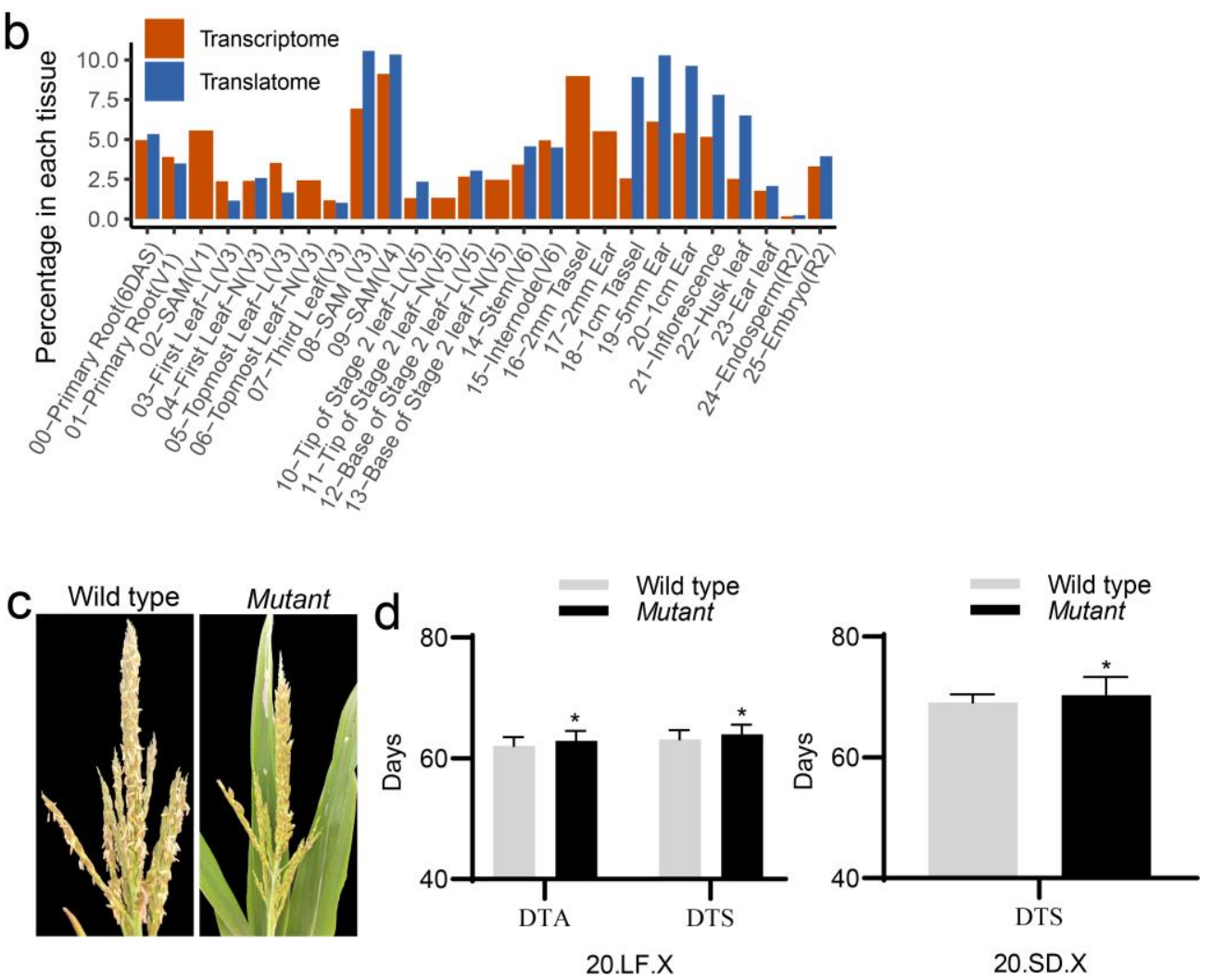

1059 
WT: GCTAAACCAGTTATAAATGCAGG(N $\left.\mathrm{N}_{\mathrm{SES}}\right)$ GTGACTCCACTGCGGTCT-GCGGG

Zm000010021291-1: GCTAAACCAGTTATAAGTGCAGG( $\left.\mathrm{N}_{555}\right)$ GTGACTCCACTGCGGTCT-GCGGG

Zm000010021291-2: GCTAAACCAGT------TGCAGG(N $\left.\mathrm{N}_{555}\right)$ GTGACTCCACTGCGGTCTTGCGGG Red: sgRNAs, green: substitution, blue: insertion and deletion

b

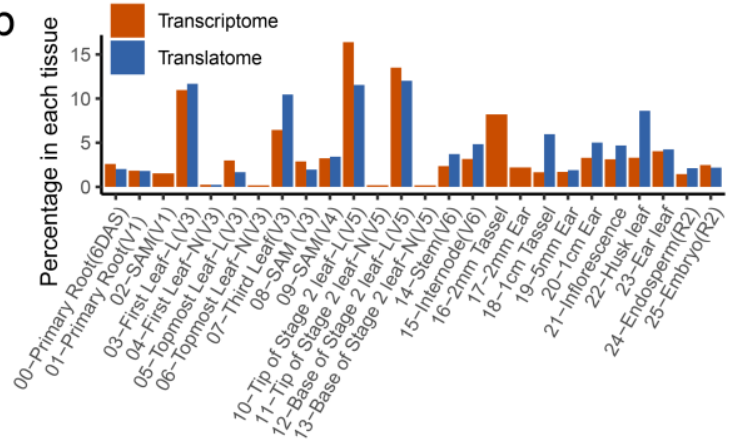

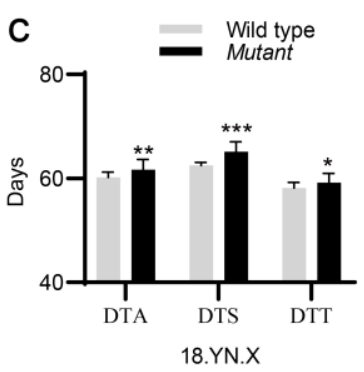

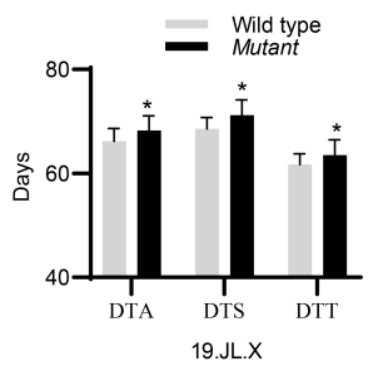

1072 Supplementary Fig. 23. Functionally validation of predicted FT genes 1073 Zm00001d021291 by CRISPR gene editing. Legends are same to Supplementary Fig. 107417.

1075

1076

1077

1078

1079

1080

1081

1082

1083

1084 
$a_{z m 00001 d 006212}$

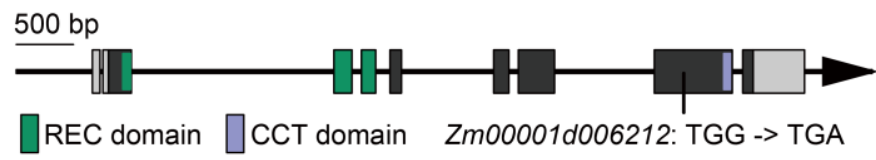

b

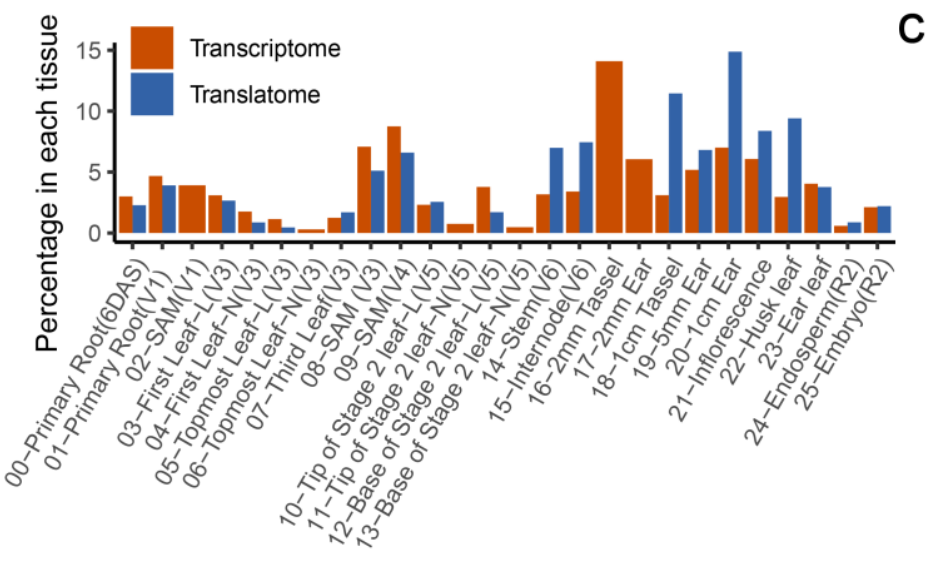

d

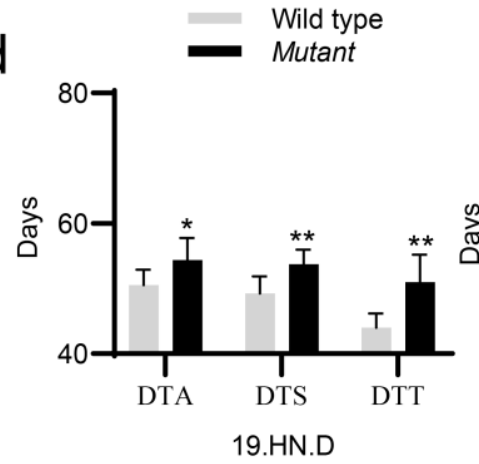

1085

1086

1088

1089

1090

1091

1092

1093

1094

1095
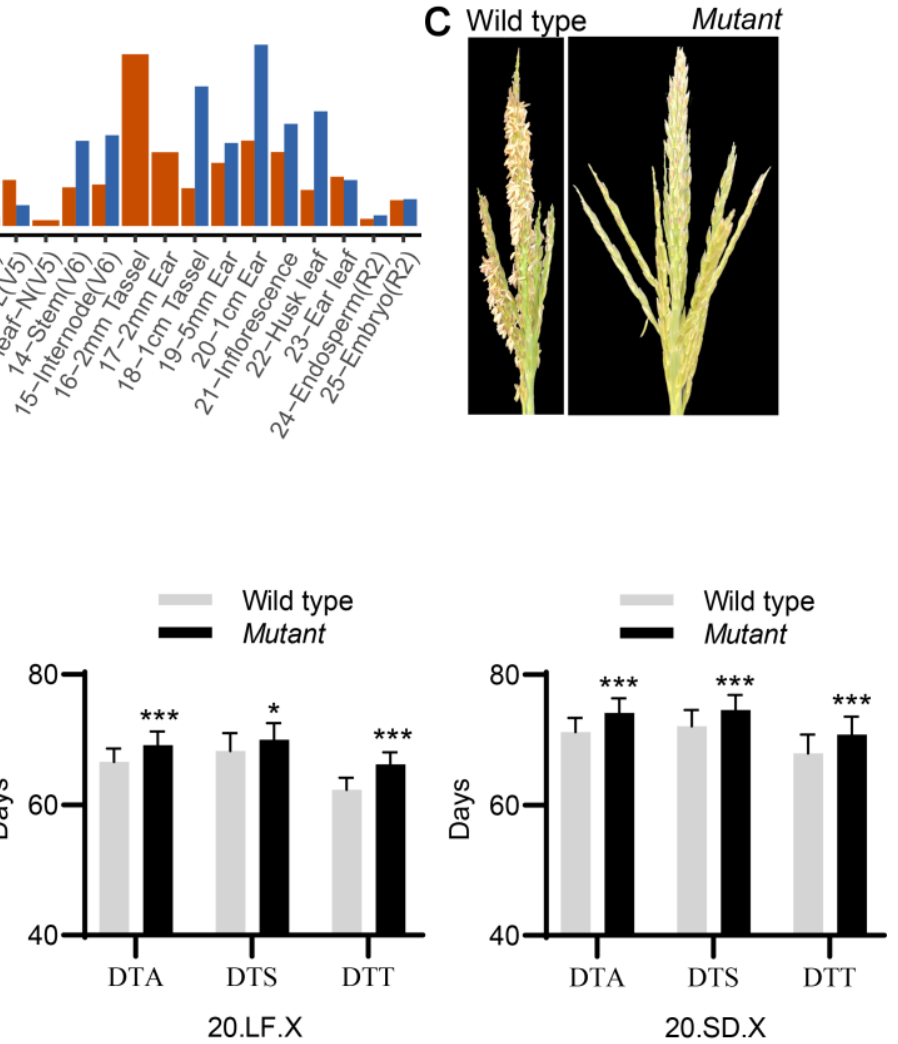

Supplementary Fig. 24. Functionally validation of predicted FT genes Zm00001d006212 by EMS mutant. Legends are same to Supplementary Fig. 17. 
a

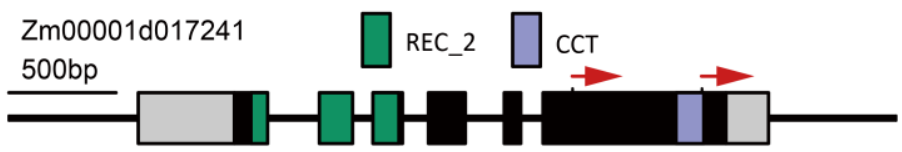

WT: GACTACAAAGAGGTGAC-AGCCGG( $\left.\mathrm{N}_{583}\right)$ GGGTGCGAGGTCAATTT-GTTAGG

Zm00001d017241: GACTACAAAGAGGTGACAAGCCGG $\left(\mathrm{N}_{583}\right)$ GGGTGCGAGGTCAATTTTGTTAGG

$\mathrm{b}$

Red: sgRNAs, blue: insertion and deletion

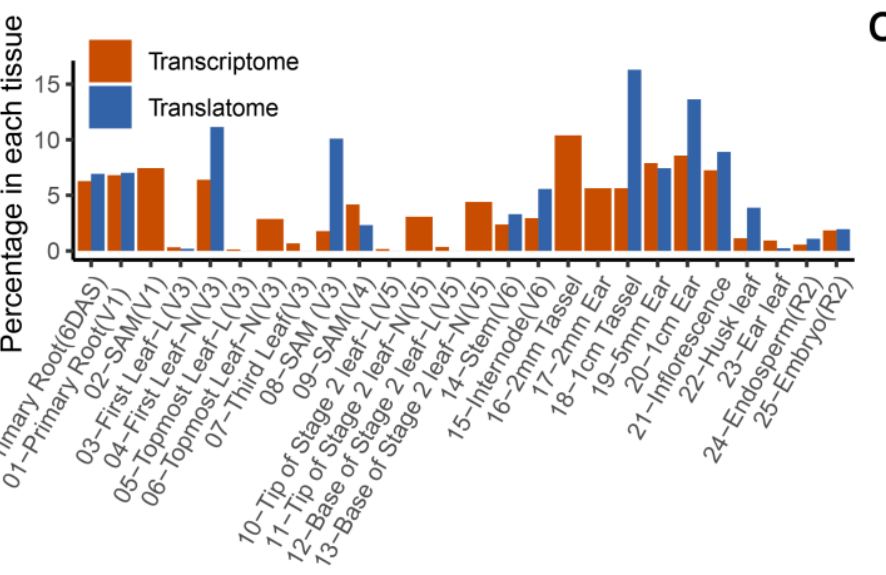

C Wild type Mutant
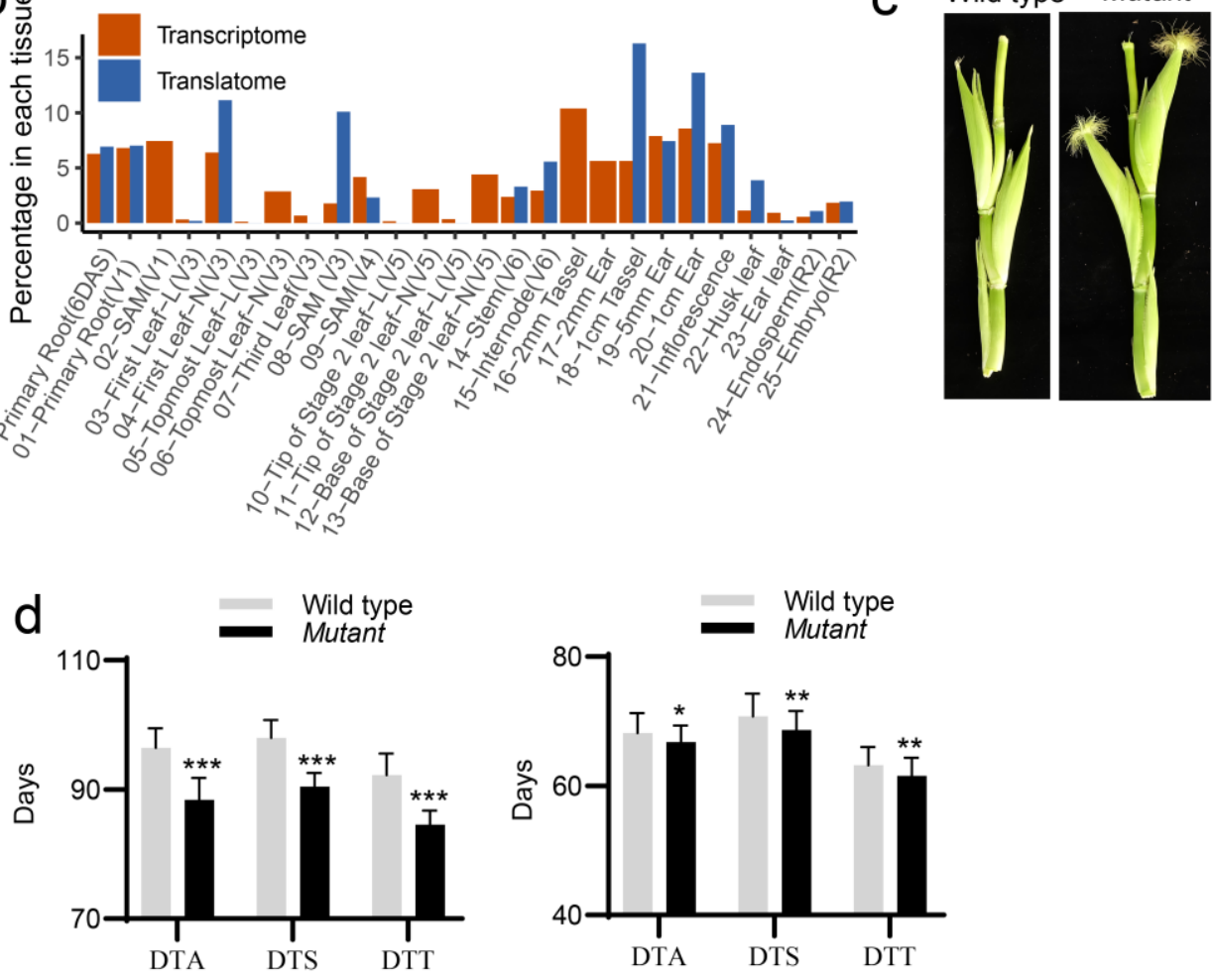

1096

18.JL.X

19.HN.D

Supplementary Fig. 25. Functionally validation of predicted FT genes 1098 Zm00001d017241 by CRISPR gene editing. Legends are same to Supplementary Fig.

1099 17. 
a zm00001d049543
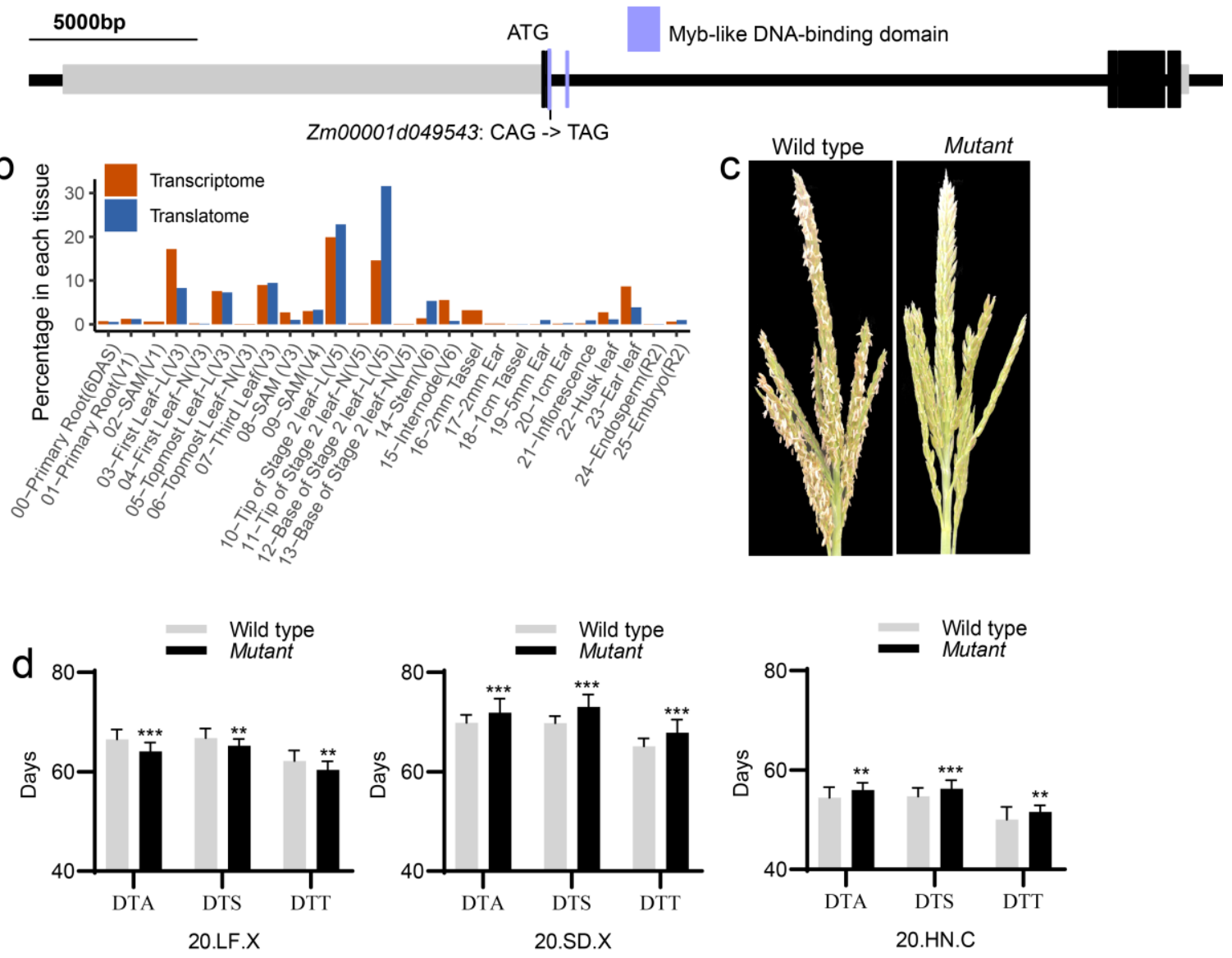

Supplementary Fig. 26. Functionally validation of predicted FT genes 1109

Zm00001d049543 by EMS mutant. Legends are same to Supplementary Fig. 17. 
a $\mathrm{Zm00001d034313}$ $500 \mathrm{bp}$

Reticulon Zm00001d034313: CGA -> TGA

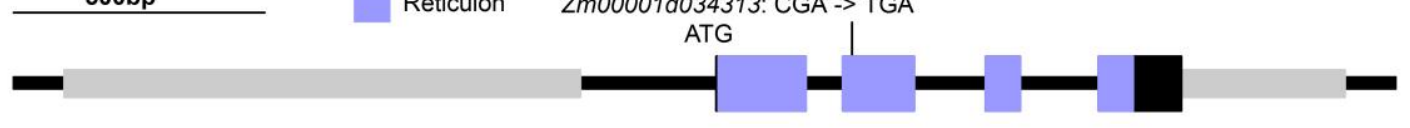

b
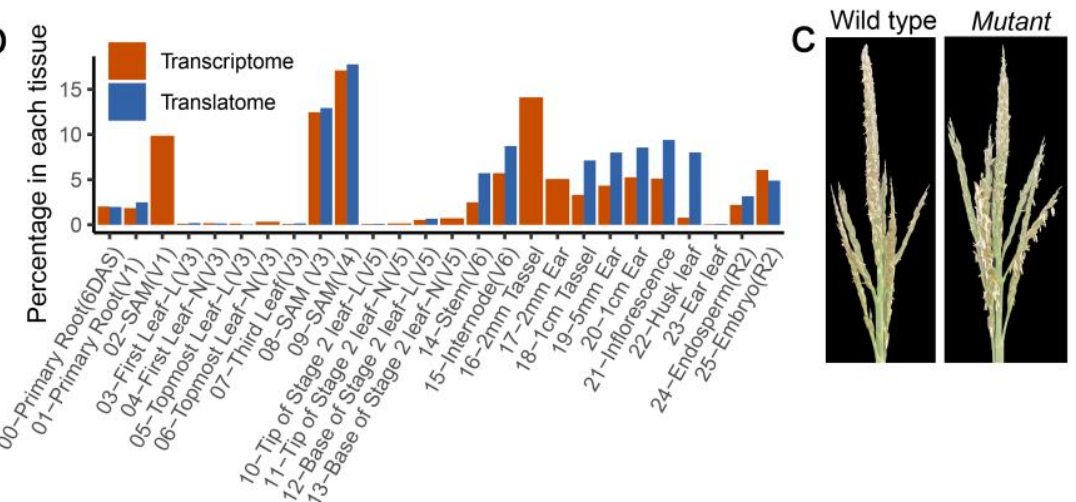

d $\quad$ Wild type
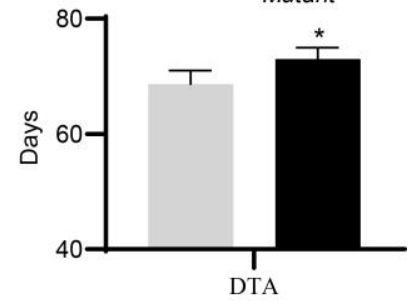

20.SD.X

1122 Supplementary Fig. 27. Functionally validation of predicted FT genes Zm00001d034313 by EMS mutant. Legends are same to Supplementary Fig. 17. 
a zm00001d006198

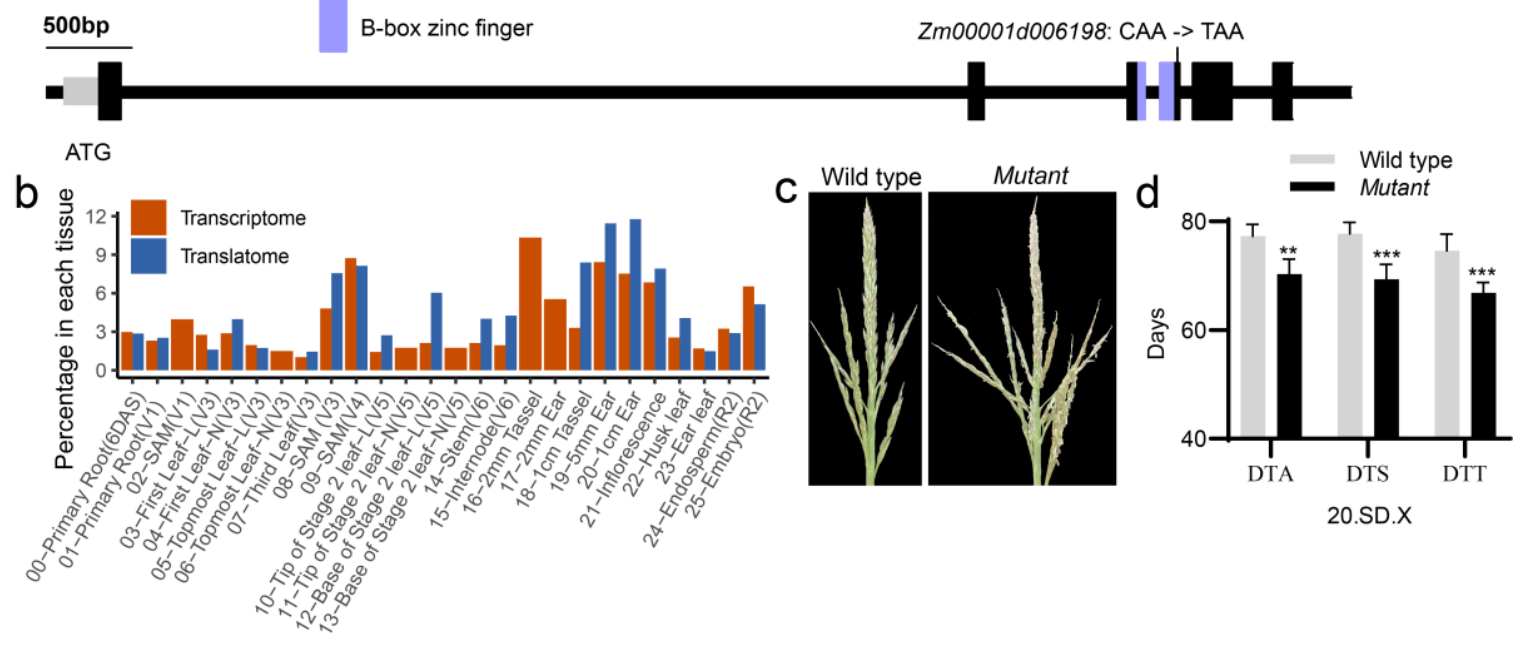

1132 Supplementary Fig. 28. Functionally validation of predicted FT genes 
$a_{z m 00001 d 010625}$

500bp

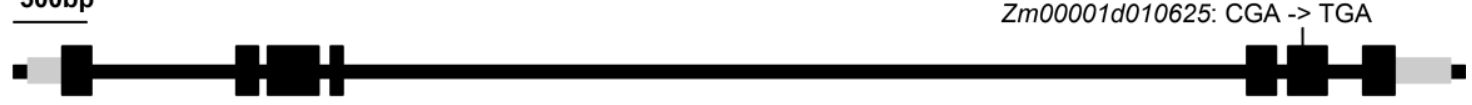

ATG

b
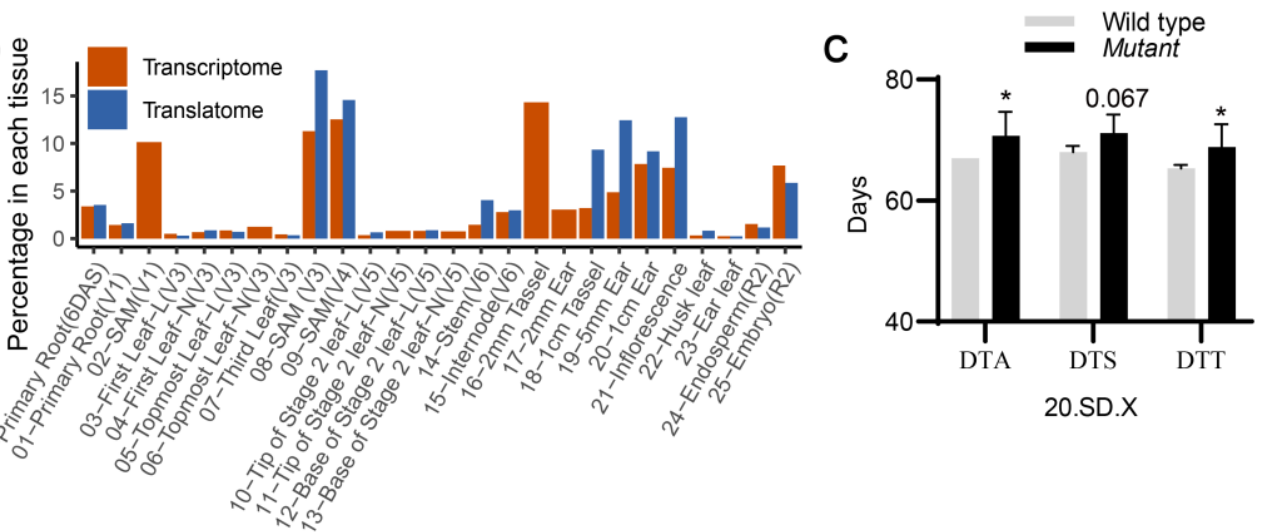

1142 Supplementary Fig. 29. Functionally validation of predicted FT genes 
a $Z m 00001 d 011748$

Zm00001d011748: CAG -> TAG

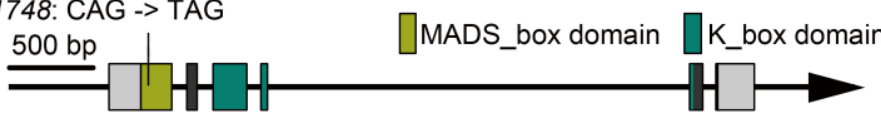

b

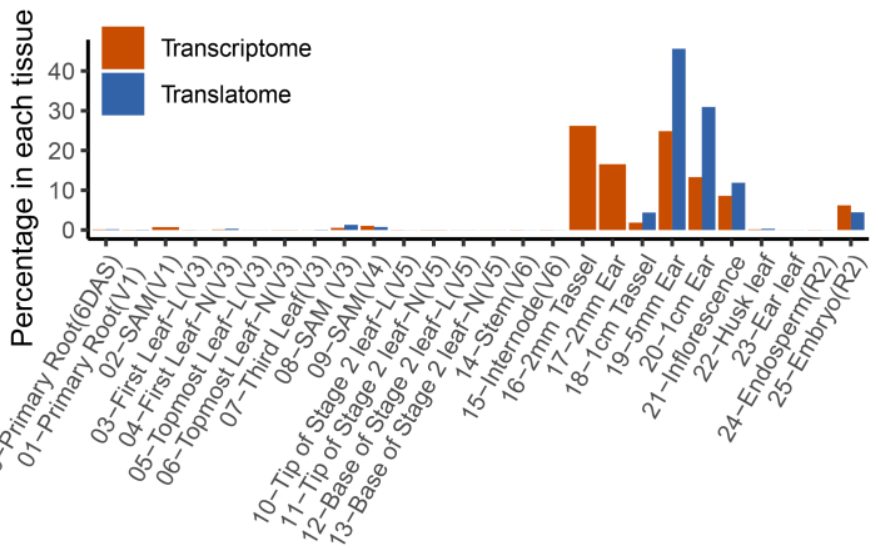

C
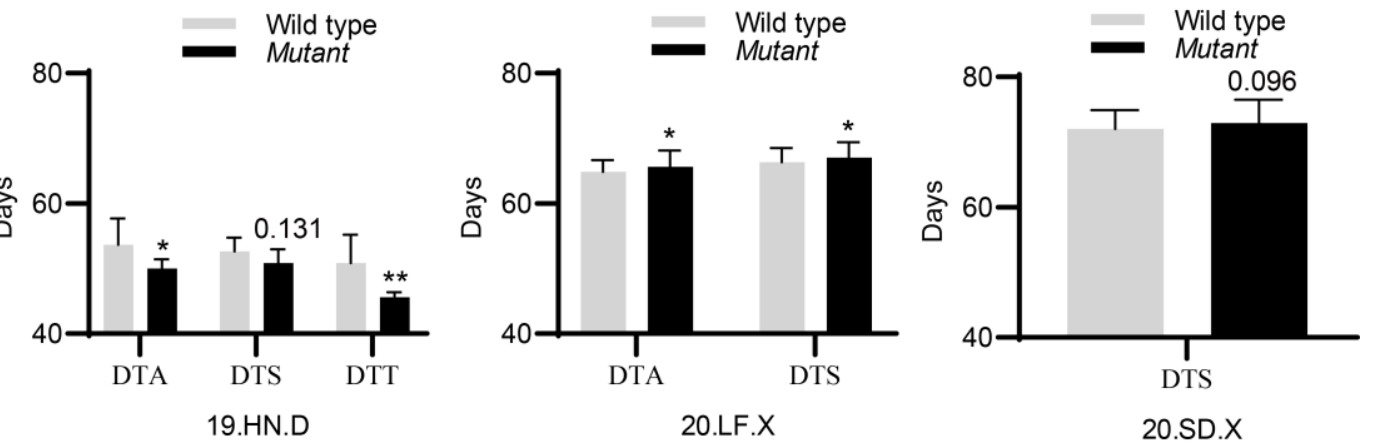

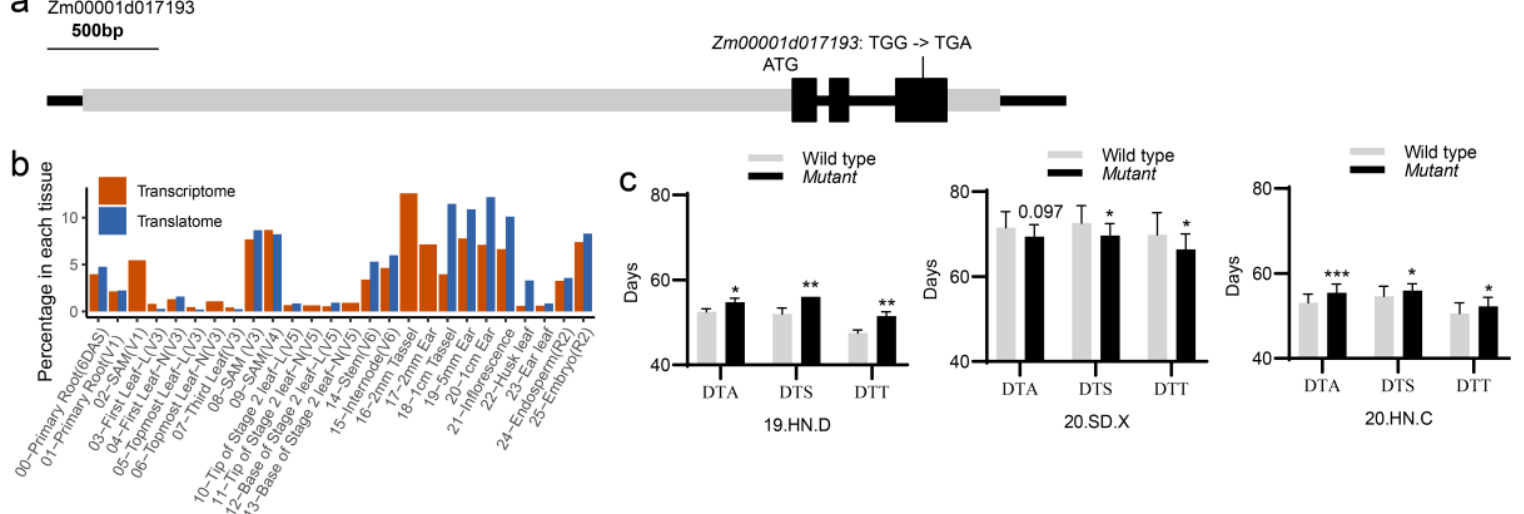
$a_{\mathrm{Zm} 00001 \mathrm{~d} 032894}$
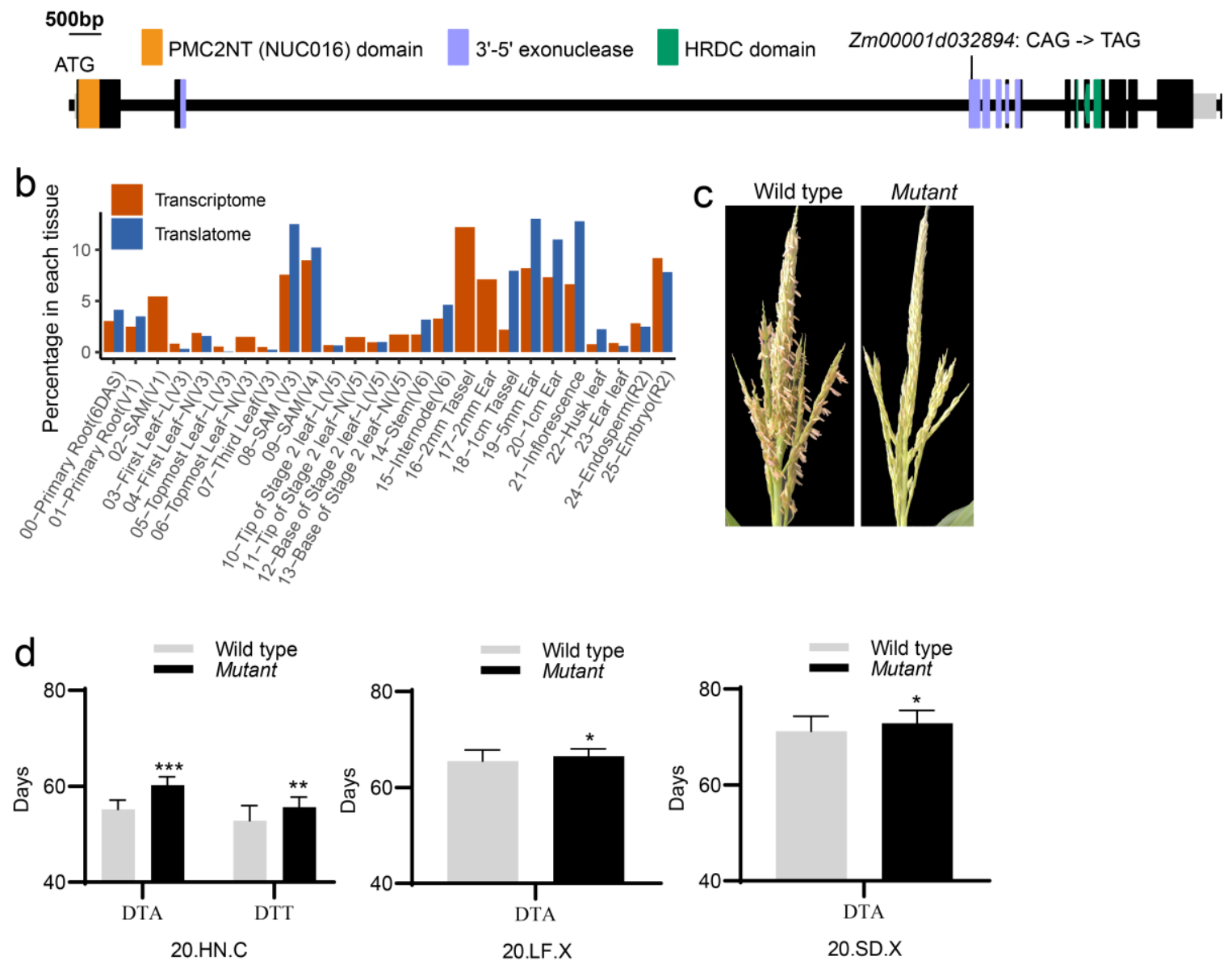


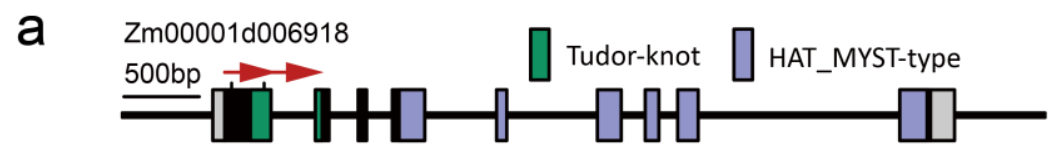

WT: GAGAACGGCTCCTCCGCTGCCGG(N $\left.\mathrm{N}_{147}\right)$ GCGCGACCAGAAGCTCCACCCGG Zm00001d006918-1: GAGAACGGCTCCTC(CGCTGCCGG...GGGCTCCGC)GTGCTCTT 216bp del Zm00001d006918-2: GAGAACGGCTCCTC(CGCTGCCGG...GCGCGACCAGAAGCTCC)ACCCGG 179bp del
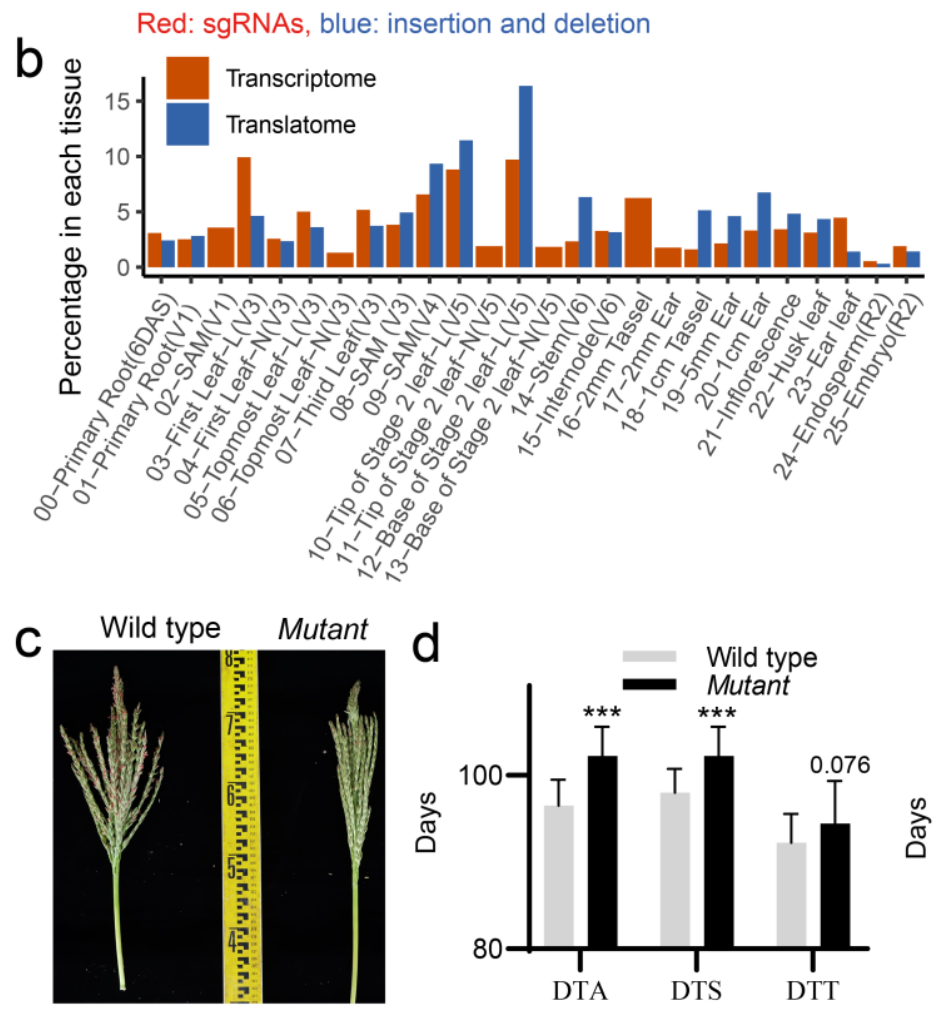

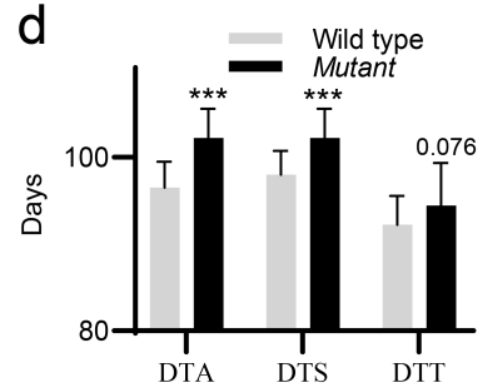

18.JL.X

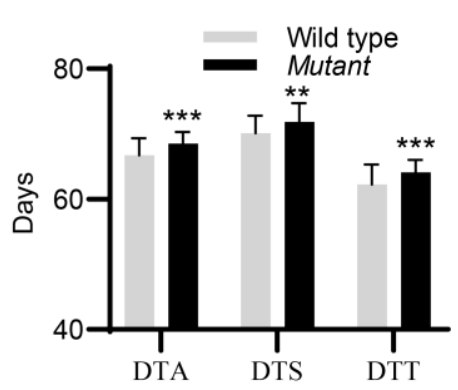

19.HN.D

1188 Supplementary Fig. 33. Functionally validation of predicted FT genes 1189 Zm00001d006198 by CRISPR gene editing. Legends are same to Supplementary Fig. 
a $z m 00001 d 006131$

$500 \mathrm{bp}$

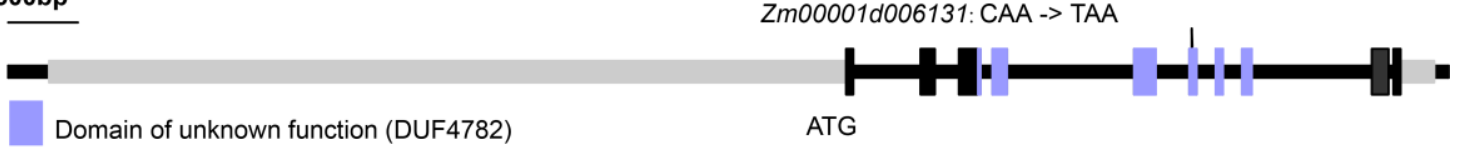

b
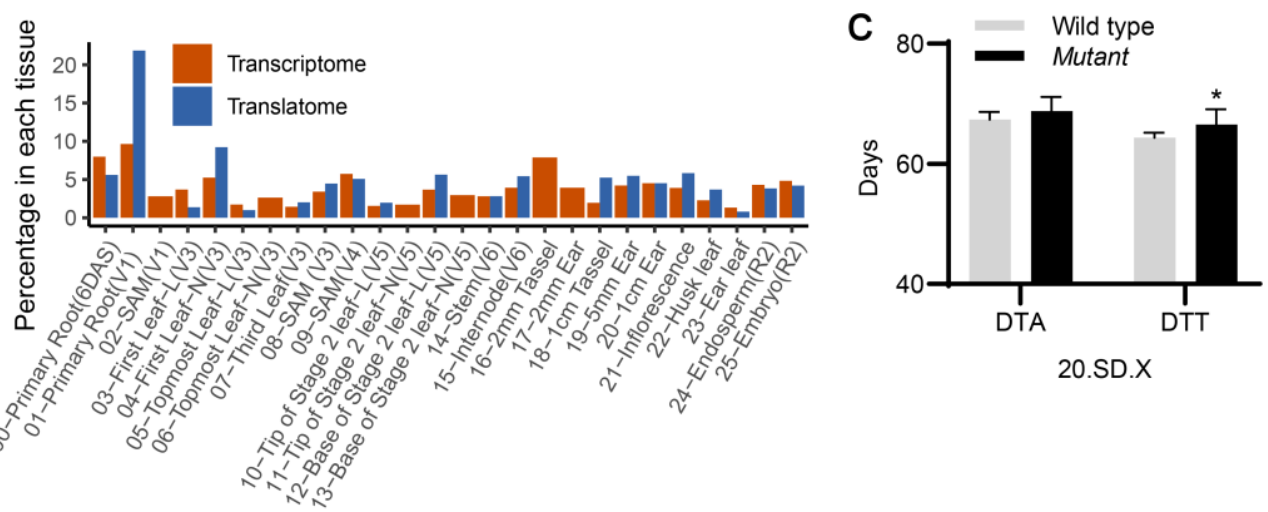

1199

Supplementary Fig. 34. Functionally validation of predicted FT genes 


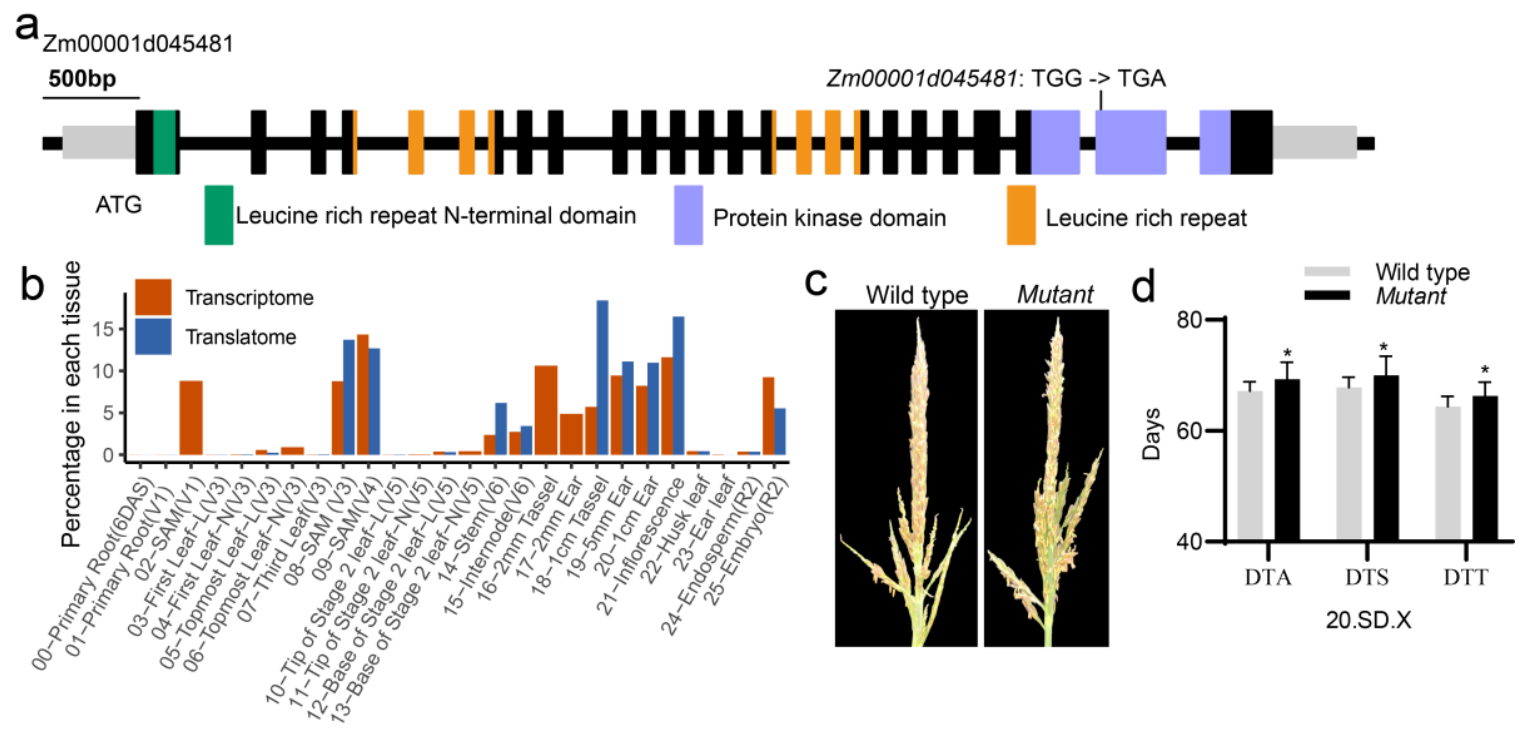

1217 Supplementary Fig. 35. Functionally validation of predicted FT genes 1218 Zm00001d045481 by EMS mutant. Legends are same to Supplementary Fig. 17. 
a

Zm00001d033680

$500 \mathrm{bp}$

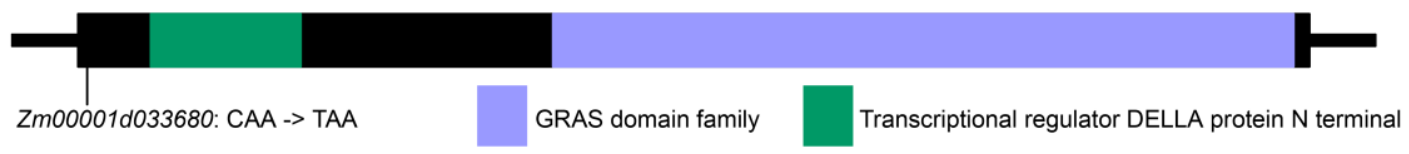

b
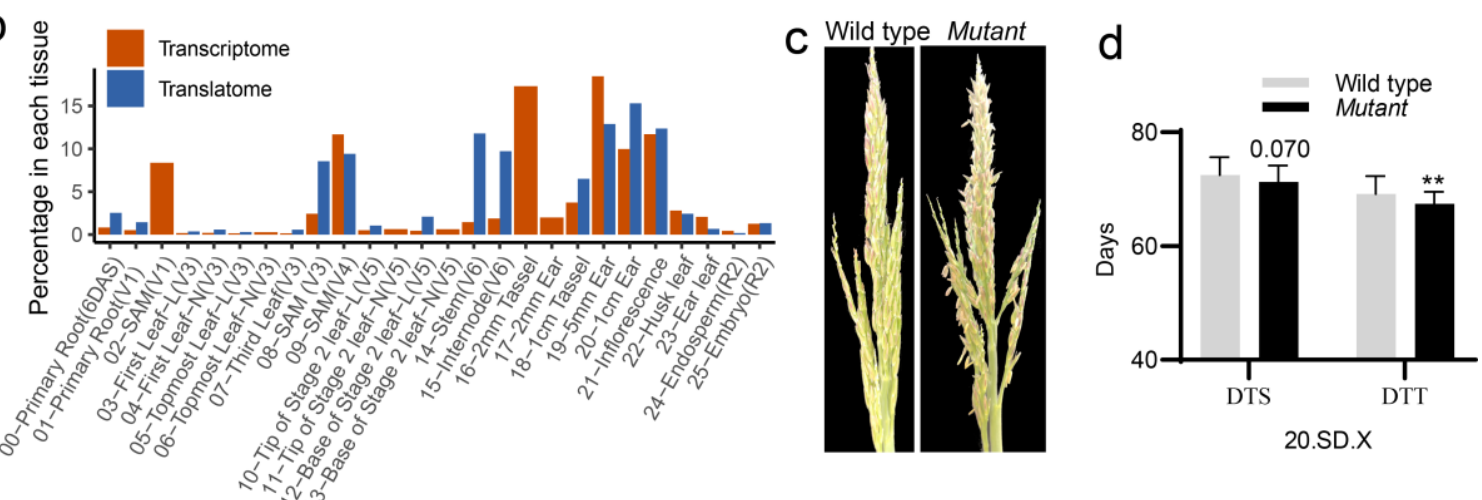
a ZmSPL6: Zm00001d042319

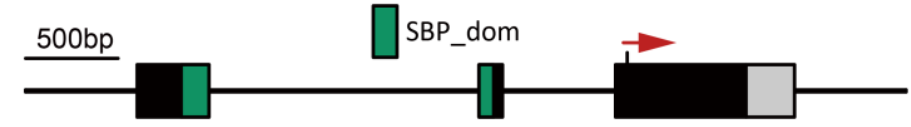

WT: GGAACAGCCTAAATGGTC-GGTGG

zmsp/6: GGAACAGCCTAAATGGTCCGGTGG

Red: sgRNAs, blue: insertion and deletion

b
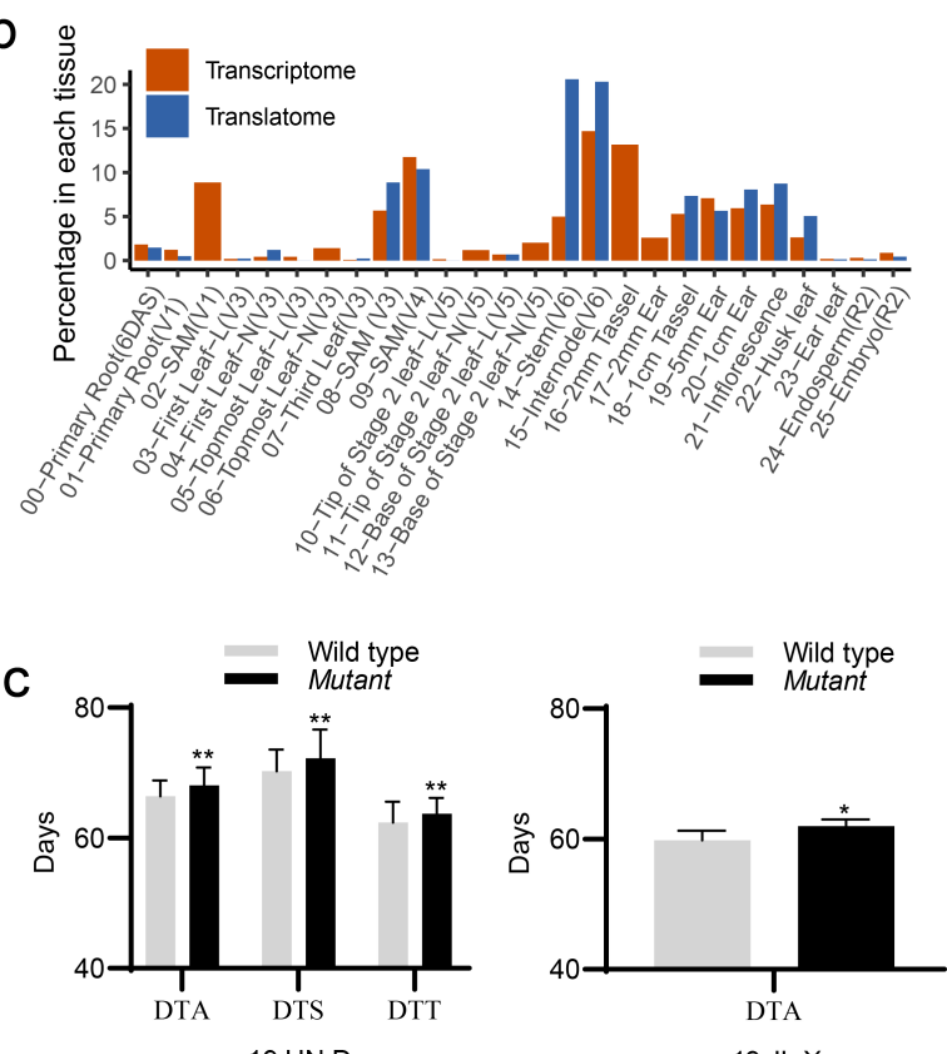

19.JL.X

1240 Supplementary Fig. 37. Functionally validation of predicted FT genes 
a zm00001d013863

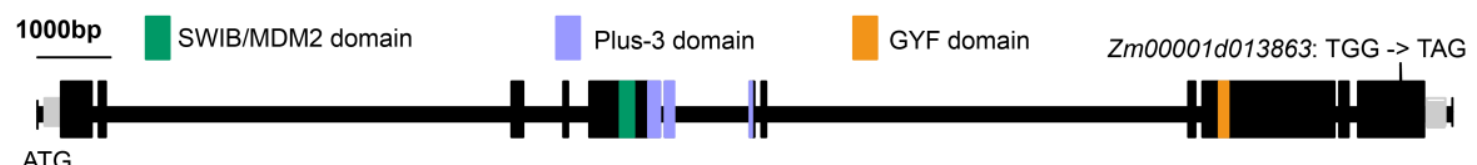

b

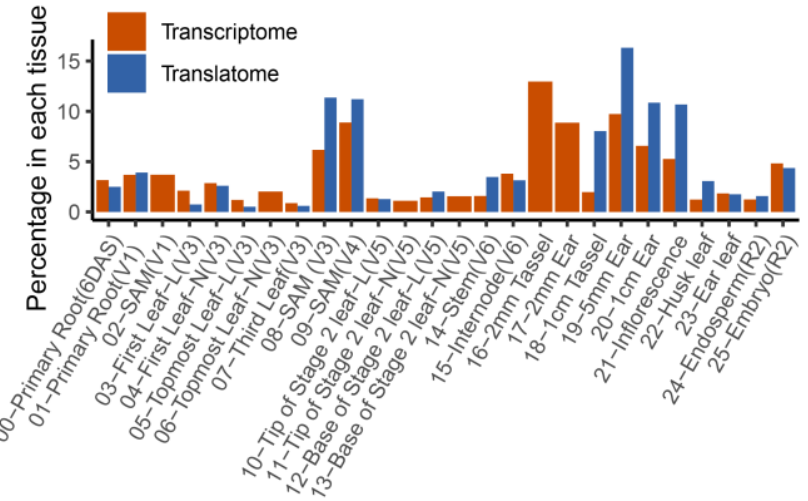

C

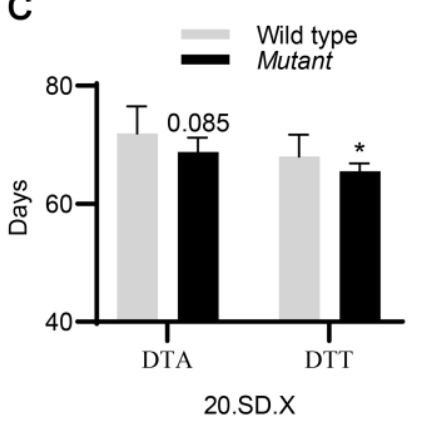

1252

1253

1254

1255

1256

1257

1258

1259
Supplementary Fig. 38. Functionally validation of predicted FT genes Zm00001d013863 by EMS mutant. Legends are same to Supplementary Fig. 17. 
a $\mathrm{Zm00001d020157}$

500bp ATG Ubiquitin carboxyl-terminal hydrolase

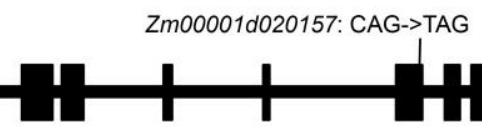

b
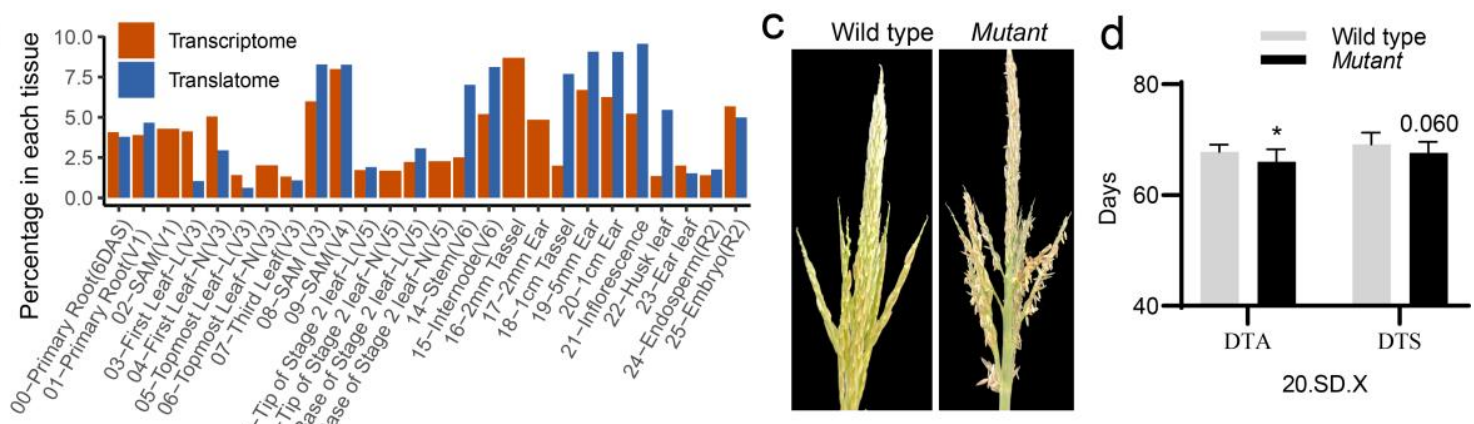

Supplementary Fig. 39. Functionally validation of predicted FT genes 
a $Z m 00001 d 034964$

500bp $\quad$ PWWP domain RNA polymerase II-binding domain Zm00001d034964: CAG -> TAG

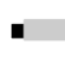

ATG

b

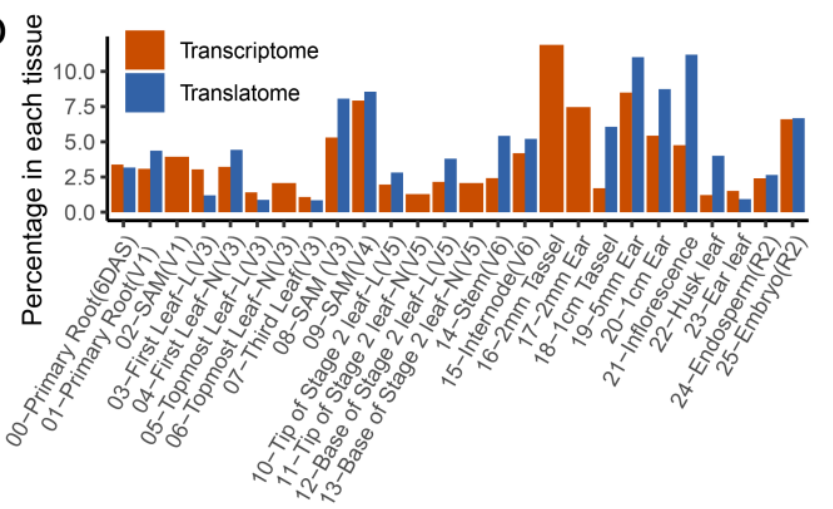

C Wild type

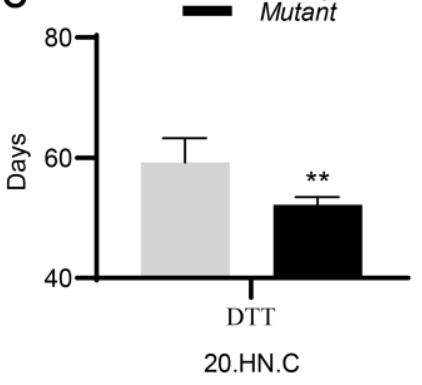

1272 Supplementary Fig. 40. Functionally validation of predicted FT genes 1273 Zm00001d034964 by EMS mutant. Legends are same to Supplementary Fig. 17.

1274

1275

1276

1277

1278

1279

1280

1281 
a $\quad Z m 00001 \mathrm{~d} 036801$

Zm00001d036801: CAG -> TAG Plant phosphoribosyltransferase C-terminal

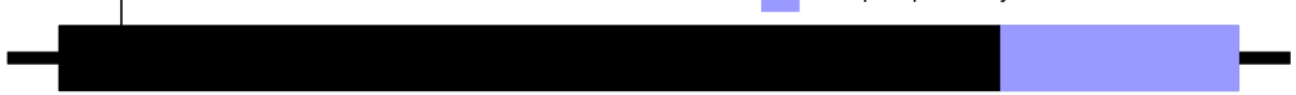

b

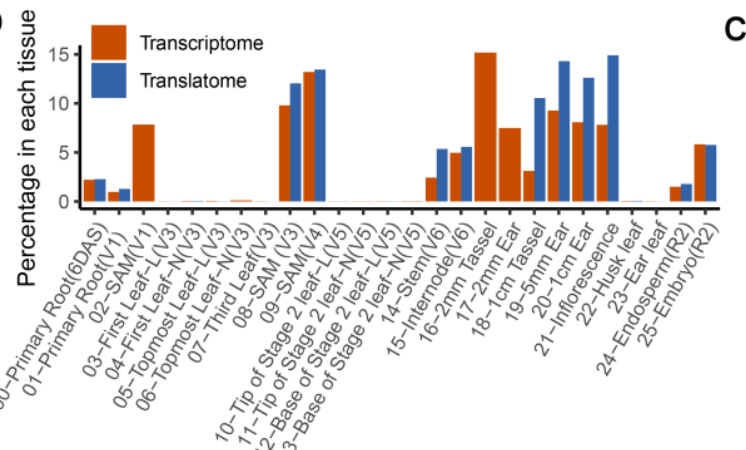

$c$

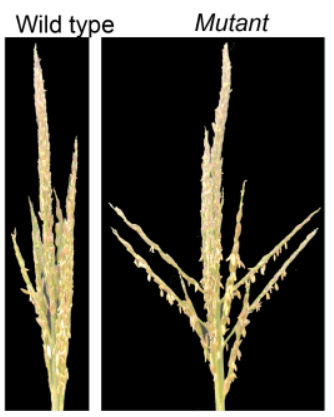

d

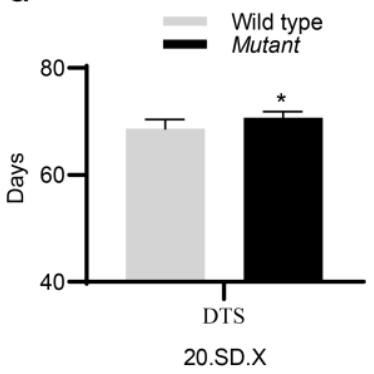




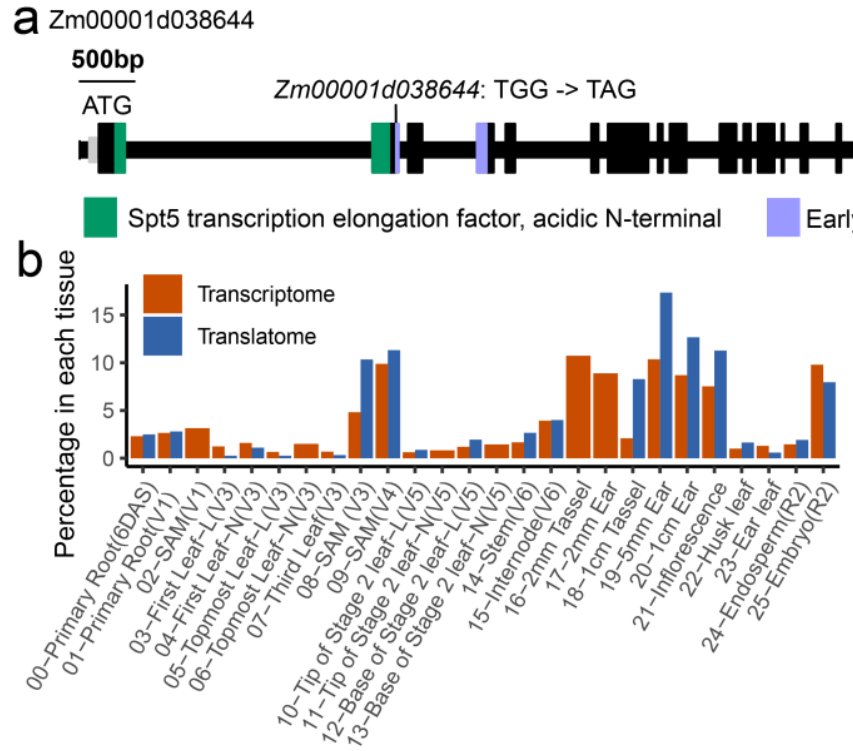

Early transcription elongation factor of RNA pol II, NGN section

Supplementary Fig. 42. Functionally validation of predicted FT genes 1295 Zm00001d038644 by EMS mutant. Legends are same to Supplementary Fig. 17. 

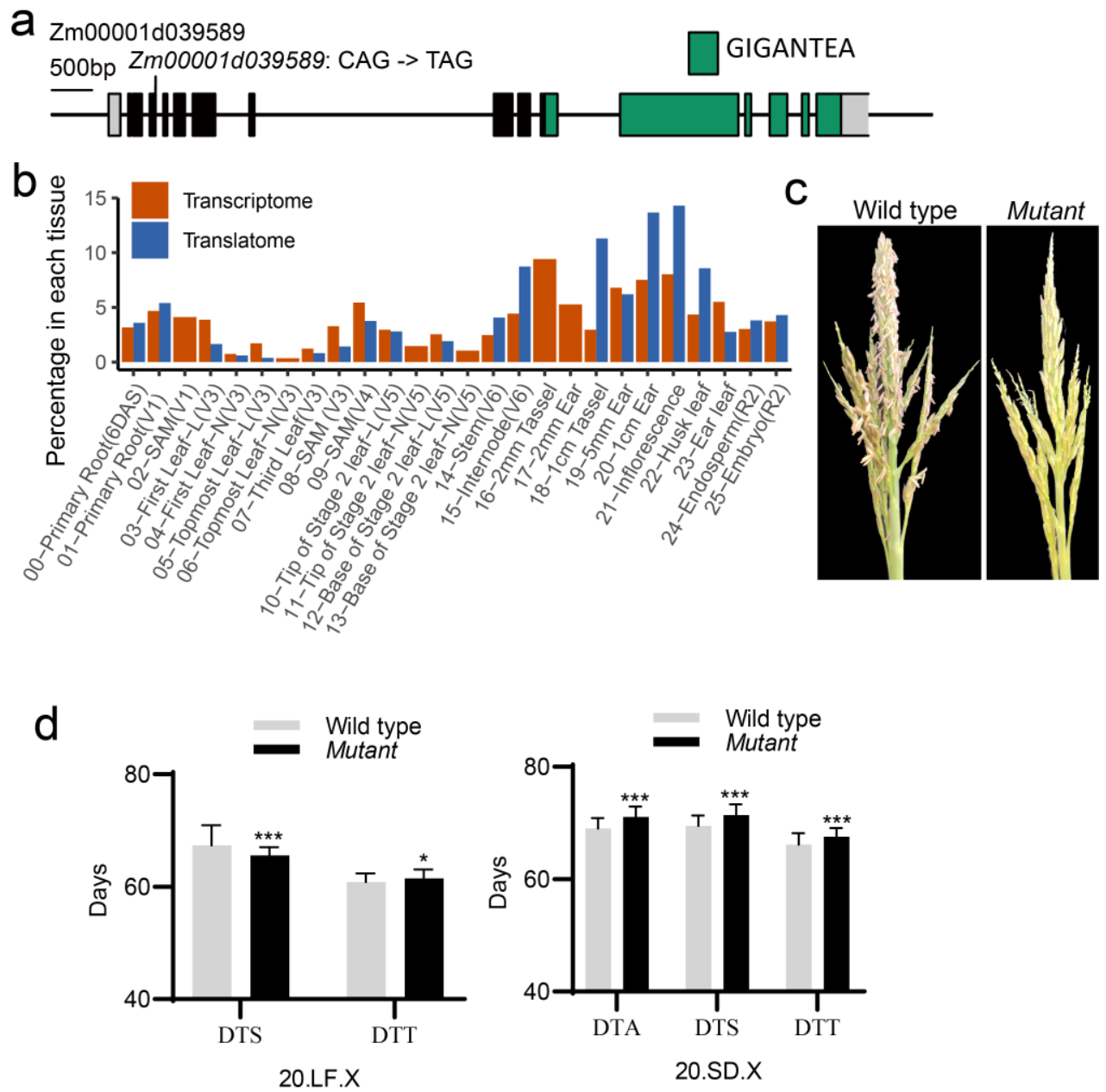

Supplementary Fig. 43. Functionally validation of predicted FT genes 1306 Zm00001d039589 by EMS mutant. Legends are same to Supplementary Fig. 17. 
$a_{Z m 00001 d 049305}$

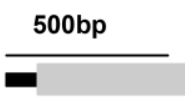

Zm00001d049305: CAG -> TAG

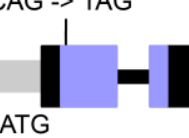

CS domain

b
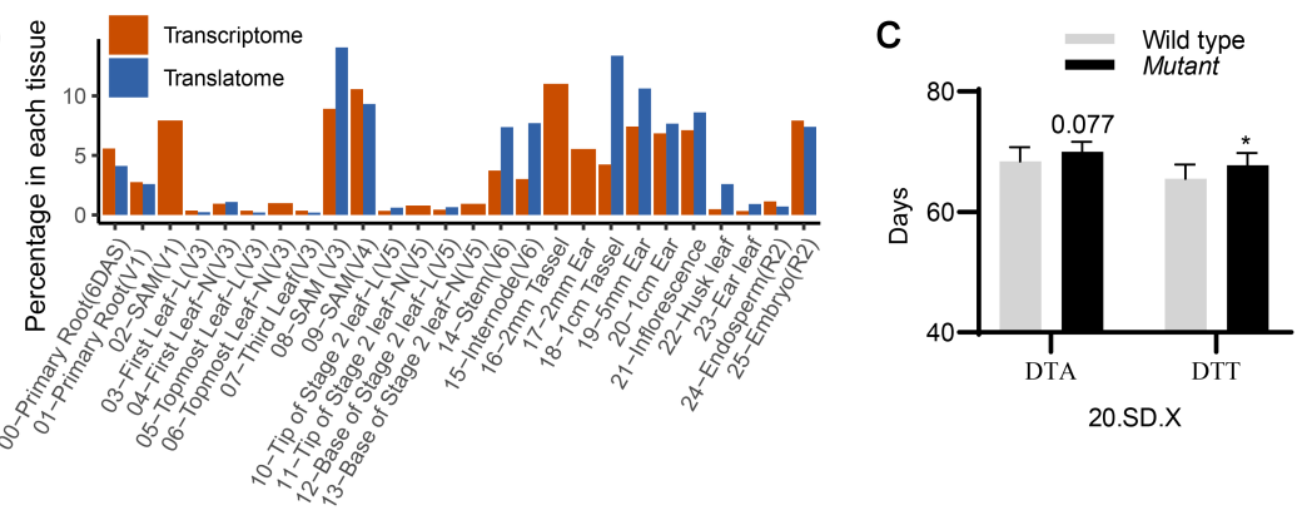

Supplementary Fig. 44. Functionally validation of predicted FT genes 
$a_{Z m 00001 d 030968}$

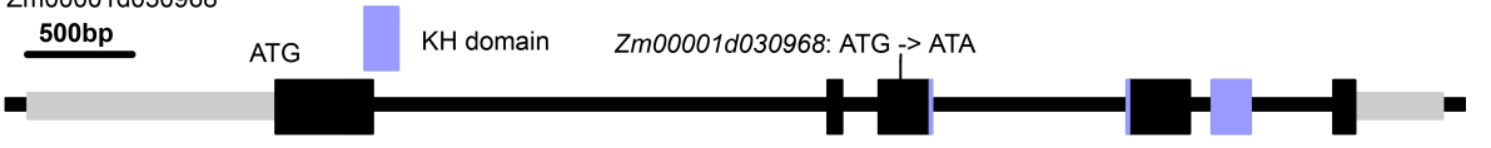

b
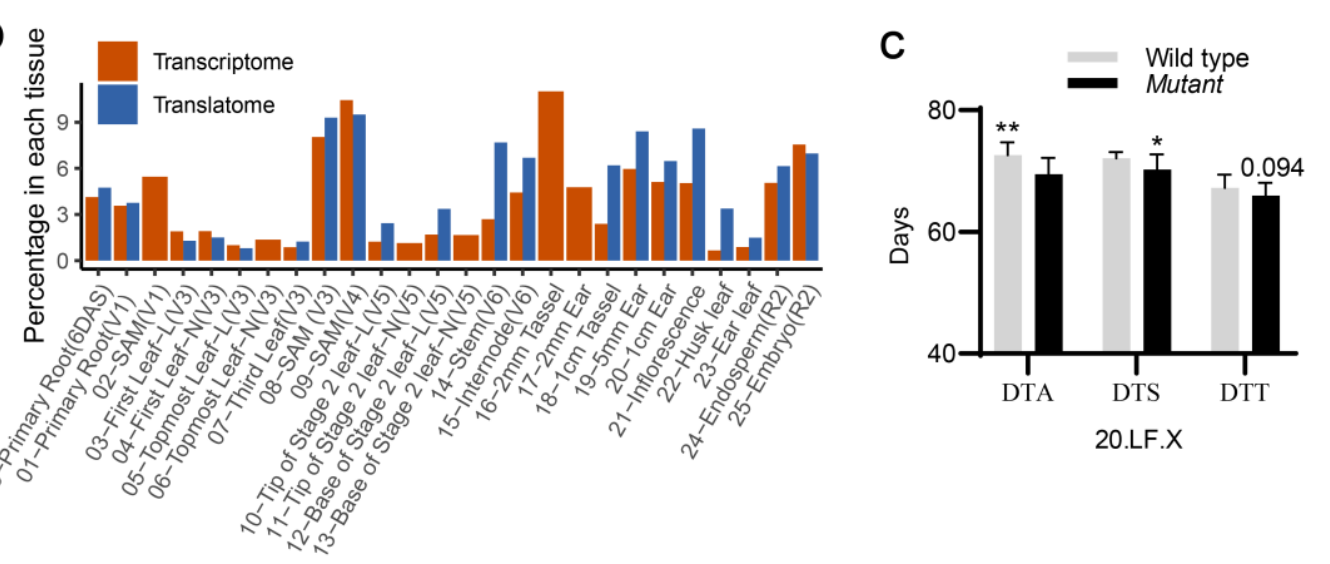

1327

Supplementary Fig. 45. Functionally validation of predicted FT genes 
a

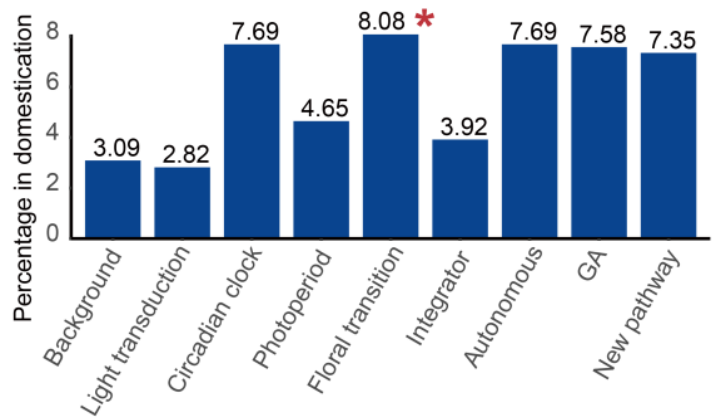

b

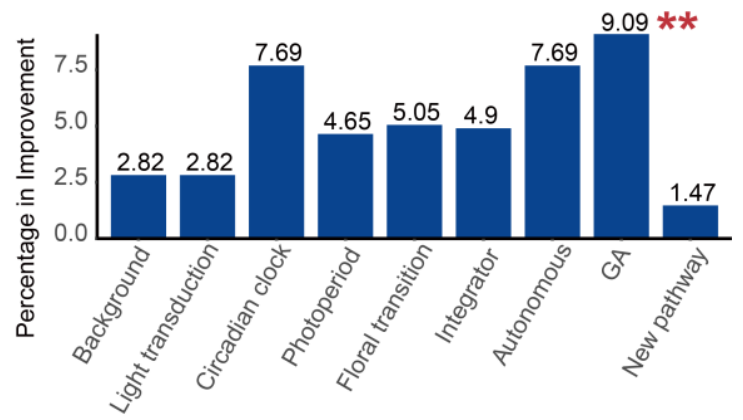

1345

1346

1347

1348

1349

1350

1351

1352

1353

1354

1355

1356

1357

1358

1359

1360

1361

1362

Supplementary Fig. 46. Percentage of genes from eight pathways enriched to selection target groups of domestication and improvement in maize. *, and ** indicate Pvalues $<0.05,0.01$ by Chi-square enrichment test, respectively. 


\section{Supplementary Tables}

1364 Supplementary Table 1. Detailed transcriptome dataset collected on the whole lifespan 1365 of reference maize inbred B73 in our study.

1366 Supplementary Table 2. Number of interactions at different layers of genetic information 1367 flow for different confidence levels.

1368 Supplementary Table 3. Eight distinct tissues for the construction of 8 bait and 8 prey 1369 cDNA libraries were applied for interaction screens of library $v s$. library mating using the 1370 RLL-Y2H system.

1371 Supplementary Table 4. All protein-protein interactions detected across different matings 1372 and different tissues.

1373 Supplementary Table 5. Detailed information of protein-protein interactions validated by 1374 BiFC.

1375 Supplementary Table 6. Detailed information of kernel related genes in maize.

1376 Supplementary Table 7. Well-known meristem development related genes in maize.

1377 Supplementary Table 8. Genes in a well-known abiotic stress related pathway.

1378 Supplementary Table 9. Detailed information of training positive and negative FT genes.

1379 Supplementary Table 10. All the predicated FT genes in maize.

1380 Supplementary Table 11. Statistical summary of FT variation for the validated FT genes.

1381 Supplementary Table 12. Eight well-curated FT pathways were uncovered in our study.

1382 Supplementary Table 13. Functional annotation of eight FT pathways.

1383 Supplementary Table 14. Primer information for the high throughput protein-protein 1384 interaction screening.

1385 Supplementary Table 15. Primer information for the genotyping of CRISPR gene editing 1386 mutations. 